\title{
SELF-CONSISTENT STUdy OF ABELIAN AND NON-ABELIAN ORDER IN A TWO-DIMENSIONAL TOPOLOGICAL SUPERCONDUCTOR
}

\author{
A Thesis Submitted to the \\ College of Graduate Studies and Research \\ in Partial Fulfillment of the Requirements \\ for the degree of Master of Science \\ in the Department of Physics and Engineering Physics \\ University of Saskatchewan \\ Saskatoon
}

By

Stephanie Goertzen

(C)Stephanie Goertzen, December 2015. All rights reserved. 


\section{PERMission TO Use}

In presenting this thesis in partial fulfilment of the requirements for a Postgraduate degree from the University of Saskatchewan, I agree that the Libraries of this University may make it freely available for inspection. I further agree that permission for copying of this thesis in any manner, in whole or in part, for scholarly purposes may be granted by the professor or professors who supervised my thesis work or, in their absence, by the Head of the Department or the Dean of the College in which my thesis work was done. It is understood that any copying or publication or use of this thesis or parts thereof for financial gain shall not be allowed without my written permission. It is also understood that due recognition shall be given to me and to the University of Saskatchewan in any scholarly use which may be made of any material in my thesis.

Requests for permission to copy or to make other use of material in this thesis in whole or part should be addressed to:

Head of the Department of Physics and Engineering Physics

116 Science Place

University of Saskatchewan

Saskatoon, Saskatchewan

Canada

S7N 5E2 


\section{Abstract}

In the past few years, there has been a burst of theoretical and experimental activity in the field of topological insulators and topological superconductors. These materials represent new states of matter which are classified by an integer invariant and exhibit topologically protected conducting edge states which appear when the material is physically terminated. One of the consequences of topological superconductivity that makes this field significant is the possible existence of Majorana fermions as an elementary excitation. Despite extensive research, however, these exotic particles remain elusive to this day.

In spite of the field of topological superconductivity being a hot topic in condensed matter research, there has been a severe lack of microscopic mean-field studies. In this thesis, we adopt a two-dimensional $s$-wave topological superconductivity model and perform selfconsistent mean-field studies in the Bogoliubov-de Gennes (BdG) formalism. As the BdG equations for topological superconductivity have high numerical demand, we implement the method of Chebyshev polynomial expansion, allowing self-consistent determination of the mean fields with high parallel efficiency and without any diagonalization of the Hamiltonian. Furthermore, we apply the recently developed Sakurai-Sugiura method to efficiently obtain the eigenpairs of the converged mean-field Hamiltonian.

We first demonstrate the differences between Abelian, non-Abelian and trivial topological order by computing the Thouless, Kohmoto, Nightingale, and den Nijs (TKNN) number and investigating the bulk-boundary correspondence. We then shift our focus to self-consistent studies, illustrating how the electron-phonon coupling strength should be chosen for several different parameter sets. Adding boundaries in the system, we are then able to confirm the appearance of Majorana fermions in the non-Abelian topological phase and discuss under which circumstances they appear. We also examine the dependence of the critical temperature of the system on the TKNN number and compare results with a recent momentum-space study on impurity effects in an $s$-wave topological superconductor [1]. The effects of depositing a single non-magnetic impurity in the center of the sample are also investigated. In particular, we find that in the case of odd TKNN number, the order parameter is extremely sensitive to even weak non-magnetic impurities signifying unconventional p-wave-like behaviour. 
Finally, we investigate the possible interplay of charge density waves and topological superconductivity. We show that within our model, topological superconductivity and topological charge density waves can coexist in the Abelian topological phase. Studying separately the pure superconducting state, the pure density wave state and the mixed state, we find that these three states are degenerate with the same ground-state energy just as in the conventional $s$-wave case. Upon introducing open surfaces in the system, zero-energy bound states are also found within all three states. Finally, single non-magnetic impurity effects are examined in the context of charge density waves. 


\section{ACKNOWLEDGEMEnTS}

First and foremost I would like to extend my most sincere thank you towards my supervisor Kaori Tanaka. We have shared many wonderful experiences together during my time as her student, and this thesis could not have been completed without her constant encouragement and guidance. The joy and enthusiasm she has for her research makes her a shining example of a physicist. I also wish to thank my committee members Rainer Dick, Yansun Yao and Artur Sowa for their contributions. I am also grateful to Yuki Nagai at the Japan Atomic Energy Agency for his collaboration and helpful discussions throughout this project. For their financial support, I want to thank the Natural Sciences and Engineering Research Council of Canada as well as the University of Saskatchewan. Finally, I would like to thank my family and friends for their support, most of all my partner Dan. He has patiently provided countless hours of advice throughout my studies and words cannot express how thankful I am for his never ending love and encouragement. 
For my mother, Lynda. 


\section{CONTEnTs}

Permission to Use $\quad$ i

$\begin{array}{ll}\text { Abstract } & \text { ii }\end{array}$

Acknowledgements $\quad$ iv

Contents vi vi vi vis

List of Tables $\quad$ viii

List of Figures $\quad$ ix

List of Abbreviations $\quad$ xiii

1 Introduction $\quad 1$

1.1 Topological Insulators and Topological Superconductors . . . . . . . . . . . . 1

1.2 Motivation for Research . . . . . . . . . . . . . . . . . . . . 10

1.3 Layout of Thesis . . . . . . . . . . . . . . . . . . . . . . . . . 12

2 Theoretical Background $\quad 14$

2.1 The BCS Theory of Superconductivity . . . . . . . . . . . . . . . 14

2.2 BdG Theory and the Extended Hubbard Model . . . . . . . . . . . . . . . . 20

2.2.1 The BdG Equations . . . . . . . . . . . . . . . . . 23

2.3 Charge Density Waves . . . . . . . . . . . . . . . 25

3 Numerical Methods $\quad 29$

3.1 The Chebyshev Polynomial Expansion Method . . . . . . . . . . . . . . . 29

3.2 The Sakurai-Sugiura (SS) Method . . . . . . . . . . . . . . . . . . . . 34

4 Topological States of Matter $\quad 40$

4.1 The Quantum Hall System . . . . . . . . . . . . . . . . . . . . 40

4.2 The TKNN Number and $Z_{2}$ Index . . . . . . . . . . . . . . . . . . . . 43

4.2.1 The Berry Phase . . . . . . . . . . . . . . . . . . . 43

4.2 .2 The TKNN Number . . . . . . . . . . . . . . . . . . 46

4.2 .3 The $Z_{2}$ Invariant . . . . . . . . . . . . . . . . . . . 47

4.2.4 The Periodic Table of Topological Insulators and Superconductors . . 50

4.3 Edge States and Majorana Fermions . . . . . . . . . . . . . . 52

5 Two Dimensional Topological Superconductivity $\quad 60$

5.1 Topological S-Wave Superconductivity in Two Dimensions . . . . . . . . . 60

5.1.1 Self-consistent Studies of Topological Superconductivity . . . . . . . . 71

5.1 .2 The Effects of Temperature . . . . . . . . . . . . . . . 77 
5.1.3 The Effects of a Single Non-magnetic Impurity . . . . . . . . . . 80

6 Charge Density Modulations and Topological Superconductivity 83

6.1 The Pure Density Wave State . . . . . . . . . . . . . . . . . . . . . 83

6.2 The Superconducting State . . . . . . . . . . . . . . . . . . . . 88

6.3 The Mixed Superconducting Density Wave State . . . . . . . . . . . . . . 90

6.3 .1 Impurity Effects . . . . . . . . . . . . . . . . . . . . . . . . 92

$\begin{array}{llr}7 & \text { Conclusion } & 94\end{array}$

$\begin{array}{lr}\text { References } & 98\end{array}$

$\begin{array}{ll}\text { A Derivation of the TKNN Number } & 104\end{array}$

$\begin{array}{ll}\text { B Generalization of BdG Theory for Topological Superconductivity } & 107\end{array}$

C Equivalence of the Hamiltonian of TSC and the Hamiltonian of TCDW 111 


\section{LIST OF TABLES}

4.1 The ten symmetry classes of topological insulators and superconductors and their corresponding invariant. $\Theta, \Xi$ and $\Pi$ correspond to time-reversal symmetry, particle-hole symmetry and chiral symmetry, respectively [76]. . . . .

5.1 The regions of trivial, Abelian, and non-Abelian topological order as reported by Sato et al. [55] for the 2-D topological superconductor described by the Hamiltonian in (5.7). $(-1)^{\mathrm{TKNN}}=-1$ corresponds to non-Abelian topological order. . . . . . . . . . . . . . . . . .

6.1 Results of the converged order parameters and ground-state energies for each of the three TSC, TCDW and TSC+TCDW states. Just as in the case of

conventional $s$-wave superconductivity, each of the three states are degenerate with the same ground-state energy. . . . . . . . . . . . . . . 


\section{List OF FigURES}

1.1 The difference in the edge states between a trivial insulator and a topological insulator. In a topological insulator or superconductor, the eigenstates rotate in Hilbert space and become twisted in analogy with a Möbius strip being a twisted version of an ordinary closed strip. Hence there is no way to continuously deform the eigenstates of a topological insulator to that of an ordinary insulator without making a "cut", meaning that one must close and reopen the energy gap. Although trivial insulators can have gapless edge states, these states are not robust to perturbations and can be easily gapped out. On the other hand, the edge states in a topological insulator or superconductor are very robust against disorder and perturbations that preserve the symmetries present in the system. Figure from [9]. Illustration: Alan Stonebraker. . . . .

1.2 Conductance maps of the Fe chain from the experiment performed by NadjPerge et al. [26] at various energies. The middle panel at $E=0$ indicates the presence of a zero-energy Majorana fermion localized at the end of the chain. Reprinted with permission from AAAS. . . . . . . . . . . . .

1.3 Conductance spectra at various temperatures measured on a cleaved surface of $\mathrm{Cu}_{0.31} \mathrm{Bi}_{2} \mathrm{Se}_{3}$. Below the critical temperature of $T_{c}=3.2 \mathrm{~K}$, the conductance at zero-bias becomes enhanced with the peak growing as temperature decreases. Figure from [25].

3.1 (a) Comparison of the order parameter obtained via direct diagonalization with the order parameter obtained varying $a$ with polynomial expansion $(n=1200$, $b=0)$. (b) Comparison of order parameters varying the number of terms in the sum, $n(a=10, b=0)$. The remaining parameters are $\mu=0$ and $U=-1.5 t$. 33

3.2 Schematic diagram of the contour on $\mathbb{C}$ with the eigenvalues marked on the real axis. . . . . . . . . . . . . . . . . . . . . . . . . . . . . . . . . . . . .

3.3 (a) Eigenvalues of the BdG Hamiltonian (2.52) with $\mu=0, U=-1.5 t$ and PBC, varying the number of quadrature points, $N_{q}$. The x-axis represents the index of the eigenvalues in ascending order. (b) Relative residual for each eigenpair varying $N_{q} \ldots \ldots \ldots \ldots \ldots$

3.4 Order parameter for a $24 \times 24 s$-wave system with $\mu=0, U=-1.5 t, n=1500$, $a=10$ and $b=0$. The presence of open boundaries in both the $x$ - and $y$ directions cause Friedel oscillations across the lattice. . . . . . . . . . . .

3.5 (a) Comparison of the residuals of the 96 lowest energy eigenpairs with increasing $L$ for a $24 \times 24$ s-wave system. (b) Comparison of the residuals obtained by the SS method with and without iterative refinement for the system shown in (a) with $L=9 \ldots \ldots \ldots \ldots \ldots$. . . . . . . . . . . . . . . . . . . . . . .

3.6 Lattice size dependence of the elapsed time for the SS method with varying energy range $\rho$ for an s-wave superconductor with $\mu=0$ and $U=-1.5 t$. . . 
4.1 The dependence of the phases of the Haldane model on the parameters $M / t^{\prime}$ and $\phi$. The trivial phase is given by $\nu=0$ and the topological phase is given by $\nu= \pm 1$. Adapted from [75]. . . . . . . . . . . . . . . .

4.2 The spin-filtered helical edge states in the quantum spin Hall insulator. Here red represents spin up while blue represents spin down. . . . . . . . . . .

4.3 Edge states for the QSH system in the topological (left) and trivial (right) phases. The black blocks represent the bulk states whereas the red and blue lines represent the spin-up and spin-down in-gap edge states, respectively. The circles and squares represent different edges of the material and the dotted line marks the Fermi energy. In the topological phase, there is an odd number of edge state pairs crossing the Fermi energy whereas in the trivial phase there are none. Note that while it is possible for the edge states to cross the Fermi level in the trivial phase if the Fermi energy were higher or lower, the difference is that the number of crossings would always be even. . . . . . . . . . .

4.4 Illustration of the trivial (top) and topological (bottom) phases of the 1D p-wave wire with open boundary conditions. The small, dark blue spheres represent the Majorana operators making up each physical site. In the topological phase, we observe two dangling zero-energy Majorana modes localized at each end of the wire. . . . . . . . . . . . . . . . . . . . . .

5.1 Energy bands $\epsilon_{+}$(blue) and $\epsilon_{-}$(orange) as a function of momentum $k_{x}$ with $\mu=3.5 t$ and $\Delta=0.5 t$. (a) The Zeeman field $h=0$ and the Rashba spinorbit coupling $\alpha=0$. The two energy bands are degenerate. (b) $h=0$ and $\alpha=1.0 t$. The spin-orbit coupling splits the energy bands depending on their spin polarization. (c) $h=0.2 t$ and $\alpha=1.0 t$. The weak Zeeman field breaks TRS and opens a small gap at $k_{x}=0 .(\mathrm{d}) h=1.0 t$ and $\alpha=1.0 t$. The stronger Zeeman field opens a large gap at $k_{x}=0 \ldots \ldots \ldots$. . . . . .

5.2 Dependence of the lowest absolute bulk eigenvalue on the Zeeman field $h$ for the exact bulk spectrum given by (5.10) (blue curve), and also for an $80 \times 80$ lattice (red points). In both curves, the parameters $\mu=3.5 t, \alpha=1.0 t$, $\Delta=0.35 t$ have been chosen. . . . . . . . . . . . . . . .

5.3 Energy spectra for a $50 \times 50$ lattice with edges at $x=0$ and $x=49, \mu=-2.0 t$, and (a) $h=0$ (trivial), (b) $h=3.0 t$ (non-Abelian), (c) $h=7.0 t$ (trivial). The remaining parameters are $\Delta=1.0 t$ and $\alpha=1.0 t$. Note that there is only one non-Abelian region for $\mu=-2.0 t . \ldots \ldots \ldots$

5.4 TKNN invariant vs. $h$ for $\mu=-2.0 t, \Delta=1.0 t$ and $\alpha=1.0 t$. The transition from the trivial phase to the non-Abelian topological phase occurs at $h \approx 2.24 t$. 67

5.5 Energy spectra for a $50 \times 50$ lattice with edges at $x=0$ and $x=49, \mu=-0.5 t$, and (a) $h=0$ (trivial), (b) $h=2.0 t$ (Abelian), (c) $h=4.0 t$ (non-Abelian), (d) $h=6.0 t$ (trivial). The remaining parameters are $\Delta=1.0 t$ and $\alpha=1.0 t$. . .

5.6 TKNN invariant vs. $h$ for $\mu=-0.5 t, \Delta=1.0 t$ and $\alpha=1.0 t$. The transition to the Abelian phase occurs at $h \approx 1.12 t$ while the transition to the non-Abelian phase occurs at $h \approx 3.64 t . \ldots \ldots \ldots$. . . . . . . . . . 
5.7 Energy spectra for a $50 \times 50$ lattice with edges at $x=0$ and $x=49, \mu=1.5 t$, and (a) $h=0$ (trivial), (b) $h=2.0 t$ (Abelian), (c) $h=4.0 t$ (non-Abelian), (d) $h=6.0 t$ (trivial). The remaining parameters are $\Delta=1.0 t$ and $\alpha=1.0 t$. . .

5.8 TKNN invariant vs. $h$ for $\mu=1.5 t, \Delta=1.0 t$ and $\alpha=1.0 t$. The transition to the Abelian phase occurs at $h \approx 1.80 t$ while the transition to the non-Abelian

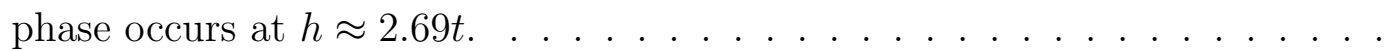

5.9 Energy spectra for a $50 \times 50$ lattice with edges at $x=0$ and $x=49, \mu=3.5 t$, and (a) $h=0$ (trivial), (b) $h=2.0 t$ (non-Abelian), (c) $h=5.0 t$ (non-Abelian), (d) $h=8.0 t$ (trivial). The remaining parameters are $\Delta=1.0 t$ and $\alpha=1.0 t$.

5.10 TKNN invariant vs. $h$ for $\mu=1.5 t, \Delta=1.0 t$ and $\alpha=1.0 t$. The transition to the first non-Abelian phase occurs at $h \approx 1.12 t$, and the transition to the second occurs at $h \approx 3.64 t \ldots \ldots \ldots \ldots$

5.11 (a) Dependence of the superconducting order parameter on the Zeeman field $h$ for a $50 \times 50$ lattice for $\mu=3.5 t, \alpha=1.0 t$ and $U=-5.0 t$. (b) Dependence of the lowest absolute eigenvalue on the Zeeman field for the system described in (a). Transition from the trivial phase to the non-Abelian topological phase occurs around $h=0.75 t . \ldots \ldots \ldots \ldots$

5.12 Value of the order parameter $\Delta$ vs. the absolute value of the coupling constant $|U|$ for a $50 \times 50$ lattice with $\mathrm{PBC}$ and (a) $\mu=-3 t, h=1.5 t, \alpha=1.5 t$, (b) $\mu=1.0, h=1.5 t, \alpha=2.0 t$ and (c) $\mu=3.5 t, h=1.0 t, \alpha=1.0 t$.

5.13 Value of the order parameter $\Delta$ vs. the absolute value of the coupling constant $|U|$ with the inclusion of the Hartree potential for a $50 \times 50$ lattice with PBC and (a) $\tilde{\mu}=-3 t, h=1.5 t, \alpha=1.5 t$, (b) $\tilde{\mu}=1.0 t, h=1.5 t, \alpha=2.0 t$ and (c) $\tilde{\mu}=3.5 t, h=1.0 t, \alpha=1.0 t \ldots \ldots \ldots \ldots \ldots$

5.14 (a) Eigenvalues of the BdG Hamiltonian (5.9) for an $80 \times 80$ system neglecting the Hartree potential with $\mu=3 t, h=1.5 t, \alpha=1.5 t, U=-5.5 t$ and surfaces at $x=0$ and $x=79$. (b) Eigenvalues for the system including the Hartree potential with $U=-4.2 t$. The remaining parameters are the same as in (a). (c) Eigenvalues for the $80 \times 80$ system neglecting the Hartree potential with $U=-4.2 t$. Selecting $U=-4.2 t$ results in a spectral gap too small for the observation of Majorana zero modes. The remaining parameters are the same as in $(\mathrm{a}) \ldots \ldots \ldots \ldots \ldots \ldots$

5.15 (a) Magnitude squared of the zero-energy wavefunction corresponding to the lowest-energy eigenvalue shown in Fig. 5.14 (a). One Majorana fermion is present on each edge of the system. (b) Magnitude squared of the wavefunction corresponding to the lowest-energy eigenvalue shown in Fig. 5.14 (c). The wavefunction is no longer localized at each edge of the system due to the exceedingly small spectral gap. . . . . . . . . . . . . . . . .

5.16 Temperature dependence of the order parameter for a $40 \times 40$ lattice with (a) $\mu=3.5 t, h=1.0 t, \alpha=1.0 t, U=-5.6 t$, (b) $\mu=3.5 t, h=2.0 t, \alpha=1.0 t$, $U=-8.0 t$, (c) $\mu=3.5 t, h=1.0 t, \alpha=1.0 t, U=-5.25 t$, (d) $\mu=3.5 t$, $h=2.0 t, \alpha=1.0 t, U=-7.38 t,(\mathrm{e}) \tilde{\mu}=3.5 t, h=1.0 t, \alpha=1.0 t$ and $U=-4.8 t$ with the Hartree potential included, and (f) $\mu=1.0 t, h=1.5$, $\alpha=2.0 t$ and $U=-4.5 t \ldots \ldots \ldots \ldots \ldots$ 
5.17 Real part of the order parameter for a single non-magnetic attractive impurity potential $V_{\mathrm{imp}}=-2.0 t$ deposited at the center of a $64 \times 64$ lattice with $\mu=3.5 t$, $h=1.0 t, \alpha=1.0 t$ and $U=-5.2 t$ corresponding to $\nu=1 \ldots . . . . . .$.

5.18 Real part of the order parameter for the case of a $64 \times 64$ lattice with $\mu=1.0 t$, $h=1.5 t, \alpha=2.0 t$ and $U=-4.445 t$ corresponding to $\nu=-2$ with the

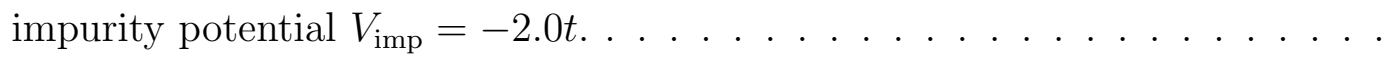

5.19 (a) Spin-up LDOS for the odd TKNN system $\nu=1$ with $\mu=3.5 t, h=1.0 t$, $\alpha=1.0 t$. (b) Spin-down LDOS for the system described in (a) . . . . . . . .

5.20 (a) Spin-up LDOS for the even TKNN system $\nu=-2$ with $\mu=1.0 t, h=1.5 t$, $\alpha=2.0 t$. (b) Spin-down LDOS for the system described in (a). In contrast to the odd TKNN case, the presence of impurity bound states are clearly distinguishable in the LDOS. . . . . . . . . . . . . . . . . . .

6.1 Energy spectra for a $50 \times 50$ system in the CDW state with edges at $x=0$ and $x=49$ with parameters $\mu=0, h=1.5 t, \alpha=2.5 t$ and $\Delta_{C}=1.0 t$. . . .

6.2 (a) Spin up and (b) spin down electron density distributions for an $64 \times 64$ bulk system in the CDW state at half-filling with $h=1.5 t$ and $\alpha=t$. . . . .

6.3 (a) Spin-up and (b) spin-down local density of states at sites $(0,0),(0,1),(1,0)$ and $(1,1)$ for a $64 \times 64$ lattice in the $\mathrm{CDW}$ state with $\mathrm{PBC}, \tilde{\mu}=0, h=1.5 t$ and $\alpha=1.0 t$. The density modulations in Fig. 6.2 are reflected in the LDOS causing the LDOS to spatially alternate from site to site. . . . . . . . . .

6.4 (a) Spin-up and (b) spin-down cross section of the electron density distribution at $y=40$ and $y=41$ for an $80 \times 80$ system in the topological CDW state with $\tilde{\mu}=0, h=1.5 t, \alpha=2.5 t$ and coupling constant $U=-4.0 t . \ldots . . .$.

6.5 Low-energy eigenvalues for Hamiltonian (6.3) for the system described in Fig. 6.4. The presence of edges causes the appearance of in-gap states in the TCDW state just as in the TSC state. . . . . . . . . . . . . . . . .

6.6 Spin-up and spin-down site-averaged density of states for an $64 \times 64$ bulk superconducting system with $\tilde{\mu}=0, h=1.5 t$ and $\alpha=1.0 t . \ldots . . .$.

6.7 Superconducting order parameter for the pure TSC state with $\tilde{\mu}=0, h=1.5 t$, $\alpha=2.5 t, U=-4.0 t$ and edges at $x=0$ and $x=79 \ldots \ldots$. . . . . . .

6.8 (a) Spin-up and (b) spin-down electron density distributions for an $80 \times 80$ lattice in the topological SC+CDW state at half-filling with $h=1.5 t$ and $\alpha=2.5 t$. The presence of edges at $x=0$ and $x=79$ causes two zero-energy Majorana modes to appear at each edge. . . . . . . . . . . . . . . . .

6.9 (a) Spin-up and (b) spin-down electron density distributions for a $51 \times 51$ lattice with $\tilde{\mu}=0, h=1.5 t, \alpha=2.5 t, U=-4.0 t$ and PBC. In the center of the lattice, we have placed a single impurity potential $V_{\mathrm{imp}}=-1.0 t$ which results in the appearance of CDW. . . . . . . . . . . . . . . .

6.10 Superconducting order parameter for the $51 \times 51$ system presented in Fig. 6.9 with $V_{\mathrm{imp}}=-1.0 t$ at site $(25,25)$. We observe impurity effects in the order parameter quite far from the impurity site consistent with the even TKNN number results presented in Section 5.1.3. . . . . . . . . . . . . . . 


\section{List of ABBREviAtions}

$\begin{array}{ll}\text { SC } & \text { Superconductivity } \\ \text { TSC } & \text { Topological Superconductivity } \\ \text { CDW } & \text { Charge Density Waves } \\ \text { TCDW } & \text { Topological Charge Density Waves } \\ \text { BdG } & \text { Bogoliubov-de Gennes } \\ \text { BCS } & \text { Bardeen-Cooper-Schrieffer } \\ \text { DOS } & \text { Density of States } \\ \text { LDOS } & \text { Local Density of States } \\ \text { PBC } & \text { Periodic Boundary Conditions } \\ \text { OBC } & \text { Open Boundary Conditions } \\ \text { SS } & \text { Sakurai-Sugiura } \\ \text { TRS } & \text { Time Reversal Symmetry } \\ \text { STS } & \text { Scanning Tunnelling Spectroscopy } \\ \text { TMD } & \text { Transition Metal Dichalcogenides } \\ \text { vHs } & \text { van Hove Singularity } \\ \text { LOF } & \text { List of Figures } \\ \text { LOT } & \text { List of Tables }\end{array}$




\section{Chapter 1}

\section{INTRODUCTION}

\subsection{Topological Insulators and Topological Supercon- ductors}

Superconductivity (SC) is a phenomenon that was discovered completely by accident by Heike Kamerlingh Onnes more than 100 years ago in 1911. While investigating the properties of supercooled mercury, Onnes discovered that the resistance of the material abruptly dropped to zero around a critical temperature of $4 \mathrm{~K}$ and later coined this phenomenon "superconductivity". Then, in 1933 Walther Meissner and Robert Ochsenfeld found that in addition to exhibiting zero resistance, superconductors exhibit what is known as the Meissner effect - the expulsion of any applied magnetic field from the material. In the present day, we know these properties as the two key features of superconductivity. Despite the discovery in 1911, however, a microscopic theory of superconductivity was not developed until 1957 when Bardeen, Cooper and Schrieffer (BCS) proposed the so-called BCS theory of superconductivity [2]. In this breakthrough theory, BCS proposed that electrons in the material pair up to form Cooper pairs as a result of electron-phonon interactions which then condense altogether to form a quantum-mechanically coherent state. One prediction of this theory was that superconductivity caused by electron-phonon interactions should only exist in any material for temperatures up to around $30 \mathrm{~K}$. Thus the discovery of high-temperature superconductivity in La-Ba-Cu-O by Bednorz and Müler [3] in 1986 came as a complete surprise. Today, researchers strive to create superconductors with higher and higher critical temperatures in the race to discover room temperature superconductivity. The highest critical temperature $\left(T_{c}\right)$ that has been recorded to date at ambient pressure is in the $\mathrm{Hg}-\mathrm{Ba}-\mathrm{Ca}-\mathrm{Cu}-\mathrm{O}$ system at $133 \mathrm{~K}$ 
[4] and recently, a $T_{c}$ of $203 \mathrm{~K}$ at $155 \mathrm{GPa}$ was recorded in sulfur hydride [5]. Although the mechanism of high-temperature superconductivity is still not understood, high- $T_{c}$ materials, especially those with $T_{c}$ greater than the temperature of liquid nitrogen, are indispensable in a variety of technological applications.

Despite the notion of superconductivity existing for more than 100 years, it was not until the early 2000s that the concept of "topological superconductivity" was introduced. The first stepping stone towards this discovery was perhaps the seminal work by Thouless, Kohmoto, Nightingale, and den Nijs (TKNN) on the quantum Hall system [6] — now considered historically the first example of a topological insulator. In 1982, TKNN were able to show that the quantum Hall effect is not only quantum-mechanical but also topological, breaking ground in the field of topological materials. The concept of an insulator or a superconductor being "topological" means that fundamental properties of the material do not change when the material parameters are varied smoothly. Moreover, each topological insulator and superconductor are classified based on the particular topology it possesses. For the quantum Hall system, TKNN proposed that there exists a mapping from $\mathbf{k}$-space onto a Hilbert space that has a particular topology characterized by an integer, topological invariant $\nu$. We say that $\nu$ is a topological "invariant" in the sense that its value cannot change unless one closes the energy gap of the system. This invariant is now known as the TKNN number and is determined by calculating the total Berry flux in the Brillouin zone. Using the Kubo formula [6], TKNN showed that the filling factor $n$ in the quantized Hall conductance, $\sigma_{x y}=n e^{2} / h$, is in fact identical to the integer invariant $\nu$, demonstrating the topological nature of the quantum Hall system. The existence of a topological invariant is key in understanding what it means for a material to be topological. Every topological insulator and superconductor is characterized by some integer invariant in the bulk, which changes depending on the dimensionality and any symmetries present in the system such as time reversal symmetry or particle-hole symmetry. If the value of this invariant is nonzero, the system is in its topological phase and if the invariant is zero, the system is in the trivial phase. Furthermore, the topological nature of a system is encoded into its eigenstates. In a topological insulator or superconductor, the eigenstates are twisted in Hilbert space in such a way that one cannot continuously deform them into the eigenstates of a trivial system without making a "cut", meaning one has to 
close the energy gap.

In addition to the existence of an integer invariant, topological systems are often characterized by the presence of edge states that lie inside the energy gap [7]. To see that these edge states must exist in topological systems, we consider the following thought experiment: consider a topological insulator or superconductor in the topological phase surrounded by or sharing a boundary with any trivial material. The material in the topological phase will be characterized by a nonzero integer topological invariant, whereas the invariant will be zero in the trivial material. Hence across the boundary, the invariant must change from nonzero to zero and in order for this to occur, one must close the energy gap of the insulator or superconductor [8]. Hence there must exist an edge mode that crosses the Fermi level. Such edge modes are often referred to as gapless edge states. The existence of a bulk topological invariant and the presence of gapless edge states when a boundary is created are the two key features of any topological insulator or superconductor. In general, however, it is not true that trivial insulators or superconductors cannot have in-gap edge states. In special circumstances, a trivial insulator or superconductor may exhibit gapless edge states [9]: the difference is that the edge states in the trivial system are not robust against disorder and can be gapped out by perturbations. On the other hand, in the topological state the edge states are protected by the topological nature of the system and also by any other symmetries present such as time-reversal symmetry. For example, in the quantum Hall system, the edge states are chiral in the sense that on one edge, propagation occurs in only one direction meaning that there are no states available for backscattering. Hence these edge states are very robust to perturbations and are said to be topologically protected. The difference in the edge states between a trivial insulator and a topological insulator is illustrated in Figure $1.1[9]$.

Then in 2005, Kane and Mele realized that if time-reversal symmetry remains unbroken, one can still obtain interesting topological insulators, albeit ones that are not characterized by the TKNN number, but ones characterized by a new topological invariant which Kane and Mele called the $Z_{2}$ index [10]. The $Z_{2}$ index gives a classification only modulo two and so it takes on only two distinct values; zero if the system is in the trivial phase, and one if the system is in the non-trivial topological phase. In terms of edge states, if there are an 


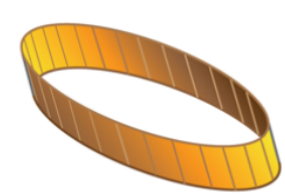

Perturbation to a trivial topological insulator

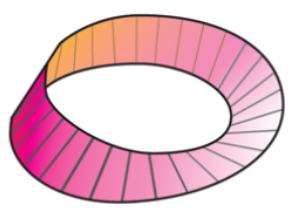

Perturbation to a topological insulator

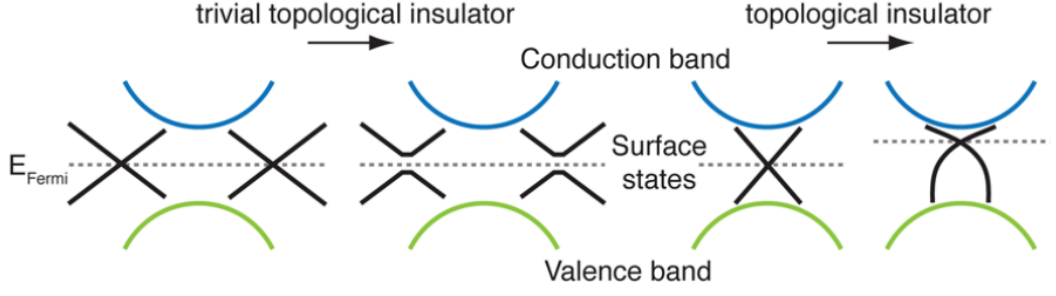

Figure 1.1: The difference in the edge states between a trivial insulator and a topological insulator. In a topological insulator or superconductor, the eigenstates rotate in Hilbert space and become twisted in analogy with a Möbius strip being a twisted version of an ordinary closed strip. Hence there is no way to continuously deform the eigenstates of a topological insulator to that of an ordinary insulator without making a "cut", meaning that one must close and reopen the energy gap. Although trivial insulators can have gapless edge states, these states are not robust to perturbations and can be easily gapped out. On the other hand, the edge states in a topological insulator or superconductor are very robust against disorder and perturbations that preserve the symmetries present in the system. Figure from [9]. Illustration: Alan Stonebraker.

odd number of pairs of edge states, the $Z_{2}$ index has a value of one, whereas if there are an even number, it has a value of zero. The system that Kane and Mele were interested in was the quantum spin Hall system - two copies of the quantum Hall system, one for spin up and one for spin down, preserving the overall time-reversal symmetry. In this system, the edge states are special in that they are helical, meaning that on a given edge, there are two spin-polarized states. Due to Kramers' theorem [11] which states that every energy eigenstate must be doubly degenerate in a time-reversal-symmetric system with half-integer spin, these helical edge states will come in Kramers' doublets. These edge states are also robust to perturbations and disorder just as the chiral edge states in the quantum Hall effect except that the perturbations must respect time reversal symmetry, otherwise a gap could open in the energy spectrum of the edge states. In this sense, we say that these helical edge states are protected by time-reversal symmetry.

In addition to introducing the concept of this new topological insulating state, Kane and Mele further proposed how to go about experimentally realizing this system using a graphene model with the inclusion of spin-orbit coupling [12]. Kane and Mele argued that 
at sufficiently low temperatures, one could observe the quantum spin Hall effect in graphene with an energy gap that is opened by the spin-orbit interaction. However, the spin-orbit interaction in graphene is quite weak and so the energy gap opened is very small, around $10^{-3}$ $\mathrm{meV}[8]$. Hence researchers began to look into materials which exhibit much stronger spinorbit interactions. This lead to the proposal of the first experimentally realizable topological insulator: mercury telluride quantum wells. First proposed by Bernevig, Hughes and Zhang (BHZ) in 2006 [13], this system was subsequently discovered experimentally by König et al. only a year later in 2007 [14].

In the situation proposed by $\mathrm{BHZ}$, the $\mathrm{HgTe}$ is sandwiched between two layers of the semiconductor CdTe to create the well structure. While CdTe has a typical band structure with the $s$-type conduction band-edge states lying above the $p$-type valence band-edge states, in HgTe the band structure is inverted where the $s$-levels lie below the $p$-levels. As a result of this inverted band structure, the electrons in the conduction band exhibit hole-like properties causing a very large spin-orbit interaction. When the HgTe layer is very thin, the quantumwell states will have normal band order; however, when the thickness of the HgTe layer exceeds some critical thickness $d_{c}$ the bands will invert. BHZ demonstrated that at $d=d_{c}$ when the $s$ - and $p$-orbital bands cross each other and the energy gap closes, the system transitions from the trivial insulator phase $\left(d<d_{c}\right)$ to the topological insulator (quantum spin Hall) phase $\left(d>d_{c}\right)$. König et al. then confirmed the quantum spin Hall phase by experimentally measuring the transport properties of $\mathrm{HgTe} / \mathrm{Hg}_{0.3} \mathrm{Cd}_{0.7} \mathrm{Te}$ quantum wells and determining $d_{c}$ to be approximately 6.3 nanometers. By tuning the chemical potential into the bulk gap, a quantized $\sigma_{x x}$ of $2 e^{2} / h$ was observed. Hence the system has finite conductance even when the Fermi level lies within the gap, signifying the presence of conducting edge states. Moreover, it was determined that these edge states were very sensitive to the presence of an external magnetic field and even a small magnetic field would destroy the conductance. These results demonstrated extensive proof of the quantum spin Hall effect in HgTe quantum wells. Shortly thereafter, the hunt for three-dimensional topological insulators began and $\mathrm{Bi}_{1-x} \mathrm{Sb}_{x}$ emerged as the first candidate in 2008 [15]. Since then, many other three-dimensional topological insulators have been discovered experimentally such as $\mathrm{Bi}_{2} \mathrm{Se}_{3}$ [16], $\mathrm{Bi}_{2} \mathrm{Te}_{3}$ [17, 18], $\mathrm{Sb}_{2} \mathrm{Te}_{3}$ $[18,19], \mathrm{Bi}_{2} \mathrm{Te}_{2} \mathrm{Se}[20,21], \mathrm{TlBiSe}_{2}[22]$ and $\mathrm{Pb}\left(\mathrm{Bi}_{1-x} \mathrm{Sb}_{x}\right)_{2} \mathrm{Te}_{4}$ [23]. 
As the field of topological insulators was being developed, topological band theory was also applied in the context of superconductivity. As superconductors exhibit a superconducting gap at the Fermi energy, the idea of topological superconductivity was a natural extension from the notion of a topological insulator. Like topological insulators, topological superconductors also satisfy the two key properties of topological materials: classification by an integer invariant such as the TKNN number or the $Z_{2}$ index, and the presence of gapless edge states when the material is physically terminated. However, unlike topological insulators, topological superconductors have the extraordinary property of being able to host Majorana fermions [24]. Majorana fermions are exotic charge-neutral particles which are their own anti-particle and arise in topological superconductors due to the intrinsic particlehole symmetry these systems possess. As a consequence of particle-hole symmetry, for every energy eigenvalue $E$ of the Hamiltonian, there exists a partner eigenvalue at energy $-E$. However, a Majorana fermion must be equal to its particle-hole conjugate at the same energy, meaning that Majorana fermions are only realized in topological superconductors at zero energy [7]. Hence in topological superconducting systems, Majorana fermions are often referred to as Majorana zero modes.

Although researchers have provided experimental evidence for the existence of Majorana fermions in various systems $[25,26,27,28]$, to date nothing conclusive can be said. Conclusive proof of the existence of Majorana fermions would not only be a breakthrough in fundamental physics, but could also make possible the realization of topological quantum computation [29]. In two dimensions, particles called anyons emerge and can obey Abelian statistics whereby the phase $\phi$ acquired under interchange of two particles ranges continuously between 0 and $\pi$

$$
\left|\psi_{1} \psi_{2}\right\rangle=e^{i \phi}\left|\psi_{2} \psi_{1}\right\rangle, \quad \phi \in(0, \pi)
$$

However, anyons can also obey non-Abelian exchange statistics in which interchange between particles is not commutative. This is the type of statistics obeyed by Majorana fermions. Non-Abelian anyons are of great interest in the field of topological quantum computation, where two well-separated anyons comprise one "qubit" - a bit in a quantum computer. When such well-separated anyon pairs are created, one proceeds to "braid" anyons from different pairs of qubits around one another to perform a computation. The resulting braid formed will 
then depend only on the topology of the overall braid and not on the intermediate details on how the braid was formed. Hence this type of computation is very robust to error as small disturbances in the paths of the anyons do not affect the computation [29]. Due to this revolutionary property, the field of topological quantum computation is rapidly growing.

As for topological superconductivity, the simplest model is the 1D p-wave wire of spinless fermions first proposed by Kitaev in 2000 [30] and now known as the Kitaev model. Since we are dealing with spinless fermions, p-wave superconductivity is required to recover the overall oddness of the pairing wavefunction in a fermionic system. In this simple toy model, Kitaev found that upon imposing open boundary conditions, two Majorana fermions would appear localized at each end of the wire. For a chain of infinite length, the interaction between these Majorana fermions would be zero. However, even though the interaction would be nonzero for a chain of finite length, Kitaev argued that the coupling would be exponentially small and hence the Majorana fermions would remain at zero energy and not be destroyed. Despite this system being proposed in 2000, it was not until 2014 when Nadj-Perge et al. experimentally provided strong evidence for the realization of a one-dimensional topological superconductor [26]. By placing a ferromagnetic iron chain on the surface of conventional $s$-wave superconducting lead, Nadj-Perge et al. aimed to make use of the proximity effect in addition to the strong spin-orbit interactions of the Rashba type at the lead surface. In the presence of strong spin-orbit coupling, this proximity effect has been predicted to induce topological superconductivity in the ferromagnetic iron chain through the tunneling of Cooper pairs from the superconductor. Using spectroscopic mapping, Nadj-Perge et al. were able to obtain the spatial structure of excitations at several energies as shown in Fig. 1.2 [26]. The middle panel at $E=0$ clearly shows a zero-energy mode localized at the end of the Fe chain, providing strong evidence for the presence of Majorana fermions and signifying that the system is indeed in the topological phase.

In two dimensions, the simplest topological superconductor is the spinless $p+i p$ superconductor which is called as such due to the superconducting order parameter having the form $\Delta_{\mathbf{p}} \propto e^{i \phi} \Delta\left(p_{x}+i p_{y}\right)$. In contrast to a conventional $s$-wave superconductor in the vortex state, a vortex in a $p+i p$ superconductor can host Majorana zero-modes. While a number of proposals have been put forth for realizing spinless $p+i p$ superconductivity in 


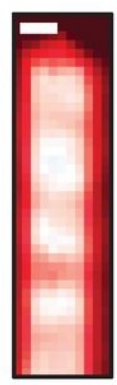

$-1.44-1.24$

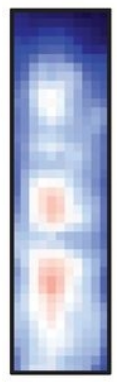

$-0.76$

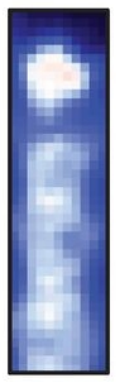

$-0.44$

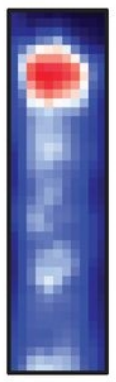

0.00

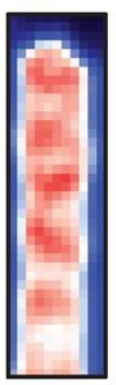

$+0.44$

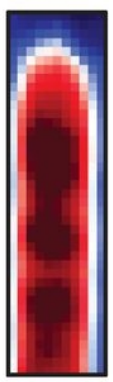

$+0.76$

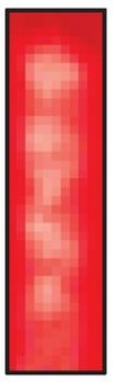

$+1.24+1.44$

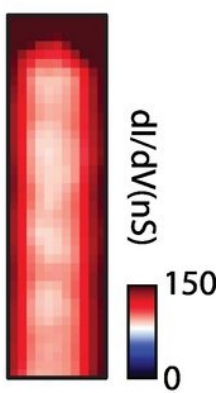

Energy (meV)

Figure 1.2: Conductance maps of the Fe chain from the experiment performed by Nadj-Perge et al. [26] at various energies. The middle panel at $E=0$ indicates the presence of a zero-energy Majorana fermion localized at the end of the chain. Reprinted with permission from AAAS.

various types of heterostructures, a promising candidate for the spinful $p+i p$ superconductor is $\mathrm{Sr}_{2} \mathrm{RuO}_{4}$ with its strong spin-orbit interactions. Experiments performed on this material including NMR Knight shift [31] and extreme sensitivity to non-magnetic impurities [32] provide strong evidence that this material exhibits spin-triplet superconductivity. In addition, it has been proposed that $\mathrm{Sr}_{2} \mathrm{RuO}_{4}$ exhibits half-quantum vortices where the vortex carries half of the magnetic flux quantum $\Phi_{0}=h c / 2 e$ [33]. Such peculiar vortices can arise in this system due to the particular structure of the order parameter. In this case, single-valuedness of the order parameter is actually achieved by a combination of the orbital part and the spin part of the order parameter as one winds around a vortex. Moreover, these half-quantum vortices bind Majorana fermions and obey non-Abelian exchange statistics, and so manipulation of vortices in systems such as $\mathrm{Sr}_{2} \mathrm{RuO}_{4}$ would be a major breakthrough in the field of quantum computation with the use of two vortices to comprise one qubit. Das Sarma, Nayak and Tewari [33] investigated the topological nature of $\mathrm{Sr}_{2} \mathrm{RuO}_{4}$ and proposed experimental techniques to stabilize the half-quantum vortices. In an experiment on a mesoscopic $\mathrm{Sr}_{2} \mathrm{RuO}_{4}$ ring, half-quantum vortices were indeed detected [34].

In addition to the 1D proximity effect model investigated by Nadj-Perge et al., the proximity effect obtained by placing a conventional $s$-wave superconductor on the surface of a 3D topological insulator proposed by $\mathrm{Fu}$ and Kane [35] is a promising method for realizing Majorana fermions. In this situation, Cooper pairs tunnel from the superconductor into the topological insulator and induce a superconducting gap in the 2D surface states. These 
surface states resemble the $p+i p$ superconductor in that creating a vortex traps a single Majorana zero mode, however, time-reversal symmetry remains unbroken. A number of experiments have been performed using this idea such as the detection of supercurrents in $\mathrm{Nb}-\mathrm{Bi}_{2} \mathrm{Te}_{3}-\mathrm{Nb}$ Josephson junctions by Veldhorst et al. [36] and scanning tunneling spectroscopy (STS) experiments performed on $\mathrm{Bi}_{2} \mathrm{Te}_{3}$ thin films grown on $\mathrm{NbSe}_{2}$ by $\mathrm{Xu}$ et al. [37]. The latter was a significant step in the search for Majorana fermions as the authors were able to create vortices on the $\mathrm{Bi}_{2} \mathrm{Te}_{3} / \mathrm{NbSe}_{2}$ interface and zero-bias conductance peaks were observed at the vortex core. However, $\mathrm{Xu}$ et al. could not conclude in their study that this was definitively a signature of Majorana fermions.

Lastly, the material that has attracted perhaps the most attention recently is the doped 3D topological insulator $\mathrm{Cu}_{x} \mathrm{Bi}_{2} \mathrm{Se}_{3}$. In this material, $\mathrm{Cu}$ atoms are intercalated into the layers of the topological insulator $\mathrm{Bi}_{2} \mathrm{Se}_{3}$ at a concentration of around $x=0.1-0.5$ and superconductivity appears below a critical temperature of $T_{c}=3.2 \mathrm{~K}$ [28]. While the exact pairing symmetry in $\mathrm{Cu}_{x} \mathrm{Bi}_{2} \mathrm{Se}_{3}$ remains unknown, it was proposed that unconventional oddparity pairing symmetry could be realized due to the particular band structure and strong spin-orbit coupling that this material exhibits. By point-symmetry group analysis, four possible pairing symmetries were proposed and subsequently narrowed down to one which was spin triplet, odd parity and naturally gave rise to time-reversal-invariant topological superconductivity [38]. Furthermore, in recent point-contact spectroscopy experiments by Sasaki et al. [28] and Ando et al. [25] with Cu concentrations of $x=0.3$ and $x=0.31$, respectively, zero-bias conductance peaks (ZBCP) were observed at low temperatures. The conductance spectra obtained by Ando et al. [25] is shown in Fig. 1.3. In both cases, the authors attribute these ZBCP to surface Andreev bound states that are Majorana fermions, providing strong experimental evidence that this material is indeed a topological superconductor. Despite this evidence, however, it remains controversial as to whether or not $\mathrm{Cu}_{x} \mathrm{Bi}_{2} \mathrm{Se}_{3}$ is in fact a topological superconductor. Follow-up low-temperature STS studies that were conducted by Levi et al. [39] presented tunnelling spectra which showed no in-gap states, suggesting that this material is simply a conventional $s$-wave superconductor. To resolve this controversy, vast improvements to the sample qualities are needed. As it stands, $\mathrm{Cu}_{x} \mathrm{Bi}_{2} \mathrm{Se}_{3}$ samples show substantial inhomogeneity and low superconducting volume fractions. However, a recent ex- 
periment by Schneeloch et al. [40] on $\mathrm{Cu}_{0.3} \mathrm{Bi}_{2} \mathrm{Se}_{3}$ found superconducting fractions of as high as $56 \%$ synthesized using a melt-growth method, and electrochemical intercalation produced superconducting fractions as high as $70 \%$ [41] showing promise for future synthesis of higher quality samples.

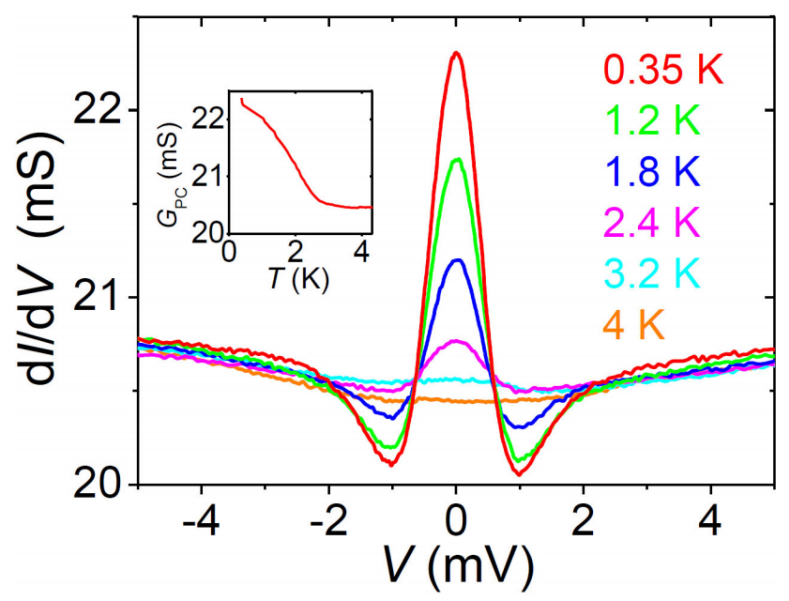

Figure 1.3: Conductance spectra at various temperatures measured on a cleaved surface of $\mathrm{Cu}_{0.31} \mathrm{Bi}_{2} \mathrm{Se}_{3}$. Below the critical temperature of $T_{c}=3.2 \mathrm{~K}$, the conductance at zero-bias becomes enhanced with the peak growing as temperature decreases. Figure from [25].

\subsection{Motivation for Research}

Several candidates for topological superconductivity have been discussed above, most of which, however, rely on unconventional $p$-wave superconductivity for the realization of the topological state. While $p+i p$ superconductivity seems to be the common theme amongst current proposals for topological superconductivity, this type of superconductivity has several downsides. First, $p+i p$ superconducting systems are very delicate and difficult to achieve experimentally as $p$-wave superconductivity is extremely sensitive to thermal fluctuations and impurities. In fact, Bauer et al. investigated the effect of thermal fluctuations on $p$ wave superconductivity and found that thermal fluctuations can have drastic effects on the topological properties of the system [42]. Moreover, $p$-wave superconducting gaps are usually very small and hence utilizing such systems for topological quantum computation could prove to be extremely difficult. With this in mind, we turn towards the possibility of non-Abelian topological order in an $s$-wave superconductor. Such a system has been proposed by Sato, 
Takahashi and Fujimoto [43] in the context of $s$-wave superfluids of ultracold fermionic atoms.

In the model proposed by Sato, Takahashi and Fujimoto, fermionic atoms are loaded in a two-dimensional optical lattice, a Zeeman field is applied and a spin-orbit interaction of the Rashba type is then generated using spatially varying laser fields [43]. As we will see in subsequent chapters, the application of a Zeeman field and the presence of Rashba spin-orbit coupling are two key ingredients for obtaining non-trivial topological superconductivity - the Zeeman field will break time-reversal symmetry and the Rashba spin-orbit interaction will essentially "freeze out" the spin degree of freedom. The authors of Ref. [43] also pointed out that although larger $p$-wave BCS gaps could theoretically be produced via a phenomenon known as Feshbach resonance in ultracold atoms, there has so far been no experimental success in creating a $p$-wave gap, whereas large $s$-wave BCS gaps can be realized easily using $s$-wave Feshbach resonance [44]. Hence realizing non-trivial topological phases in 2D $s$-wave superfluids of ultracold fermionic atoms is quite promising experimentally for studying the properties of Majorana fermions and developing topological quantum computation [43].

Adapting this 2D $s$-wave topological superconductor model, in this thesis we perform microscopic mean-field studies of 2D topological superconductivity by self-consistently obtaining converged solutions for not only the superconducting order parameter, but also the Hartree potential. To date, there have been very few self-consistent studies of topological superconductivity and to the best of our knowledge, no self-consistent mean-field study in real space has been made for the present 2D $s$-wave model. Using a Green-function formulation in momentum space, a single self-consistent study of this $s$-wave model was performed by Nagai, Ota and Machida [1] who examined the effects of non-magnetic impurities on topological superconductivity by incorporating impurities in terms of the self-energy. A very limited number of other self-consistent studies have been performed on different systems such as the work of Zhou, Gao and Wang [45] on a 2D model and the work of Qu, Gong and Zhang [46] on a 1D model. In this thesis, we present a full real-space, self-consistent study of 2D s-wave topological superconductivity in terms of the Bogoliubov-de Gennes (BdG) theory, confirming the existence of Majorana fermions in this model, studying how single non-magnetic impurities affect the system, and examining how the Hartree potential alters the topological phases. In order to accomplish this, we have implemented the Chebyshev 
polynomial expansion method $[47,48]$ which allows for efficient self-consistent determination of the superconducting order parameter and Hartree potential without any diagonalization of our large two-dimensional Hamiltonian. In addition, we have implemented a recent numerical technique proposed by Sakurai and Sugiura $[49,50]$ that enables us to effectively obtain the eigenpairs of our Hamiltonian within a desired energy range, in particular, lowest-energy eigenvalues.

Lastly, we self-consistently investigate the interplay between topological superconductivity and charge density waves - periodic modulations in the electron density of a material. Since the 1970s, there has been significant debate as to whether or not charge density waves cooperate or compete with superconductivity. To this day, the controversy still has no resolution, with many studies publishing conflicting results [51, 52, 53, 54]. Moreover, there has been no experimental work nor has there been self-consistent studies which investigate topological charge density waves, let alone the interplay between topological charge density waves and topological superconductivity. The Hamiltonian for the topological charge density wave state was proposed by Sato, Takahashi and Fujimoto in 2010 [55] who demonstrated that this Hamiltonian allowed the presence of zero-energy bound states although no self-consistent studies were performed. Recently, Scheurer and Schmalian also theoretically studied the possibility of charge density wave formation in the electron fluid formed at the 2D interface between the oxides $\mathrm{LaAlO}_{3}$ and $\mathrm{SrTiO}_{3}$, which is thought to exhibit signatures

of time-reversal-symmetric topological superconductivity [56]. Again however, the charge density wave study was brief and no self-consistent calculations were performed. The lack of self-consistent studies of two-dimensional topological superconductivity and the lack of understanding regarding how charge density waves behave in such systems provide the primary motivation for the work presented in this thesis.

\subsection{Layout of Thesis}

The intent of this thesis is to provide an introduction to topological superconductivity and to investigate how these systems behave when charge density modulations are present. As such, in Chapter 2 we begin with the general theoretical background for conventional supercon- 
ductivity and charge density waves. Chapter 3 then deals with recently developed numerical methods that allow us to efficiently perform mean-field calculations and obtain low-energy eigenvalues of our Hamiltonian. We then move into Chapter 4 which is meant to be an introduction to topological superconductivity including a description of topological invariants, edge states and Majorana fermions. In Chapter 5 we present our preliminary results with the focus being on self-consistent studies of topological superconducting systems. The main results of this thesis are found in Chapter 6 where we self-consistently study the interplay of topological charge density waves and topological superconductivity. Lastly, Chapter 7 concludes the thesis and provides a discussion of the main results obtained. 


\section{Chapter 2 \\ Theoretical BaCKGround}

\subsection{The BCS Theory of Superconductivity}

Prior to 1957, a comprehensive understanding of the mechanism behind superconductivity (SC) was much sought after. At the time, all theories proposed were phenomenological theories developed by London, Ginzburg and Landau and were only able to provide qualitative descriptions creating a strong need for a microscopic theory. Finally in 1957, Bardeen, Cooper

and Schrieffer introduced the very first microscopic theory of conventional superconductivity now known as the BCS theory of superconductivity [2]. Formulated in momentum space, the BCS theory is very successful in describing conventional or low-temperature superconductivity which originates from electron-phonon interactions. However as translational invariance is assumed, only bulk, homogeneous systems are applicable within the BCS theory.

The BCS theory is built upon the Landau theory of the Fermi liquid which states that if one starts with a non-interacting ideal Fermi gas, the single-particle excitations of the interacting system can then be constructed by adiabatically switching on interactions between particles such as the repulsive Coulomb interaction among the conduction electrons. Essentially, adiabatically switching on these interactions creates a one-to-one correspondence between an eigenstate in the ideal system and an eigenstate in the interacting one, and the key result being the existence of the well-defined Fermi surface. In addition, Landau recognized that the net effect of the interactions between electrons is to simply shift the effective mass of the electron and hence the electrons are called "quasiparticles". How one then obtains superconductivity is to include the residual electron-phonon interactions that are neglected within the Landau-Fermi liquid theory.

Working towards a microscopic theory, Cooper decided to examine the result of placing 
a pair of electrons which interact via a small attractive two-body potential above a noninteracting Fermi sea - the one-pair problem [57]. To understand the origin of the attractive potential, consider one electron moving through a lattice of positive ions. This electron will polarize the lattice as it travels, causing a second electron to interact with the polarized lattice. These two electrons then become correlated leading to an effective attractive interaction. This effective attractive interaction occurs only near the Fermi surface, however, roughly within $\pm \hbar \omega_{D}$ around the Fermi energy, where $\omega_{D}$ is the Debye frequency. The surprising result Cooper discovered was that the electrons inevitably form a bound state, no matter how small the interaction. The key is that the interaction is attractive and more importantly, that we have a filled Fermi surface. Considering this pair of electrons, we are interested only in the minimum energy of the pair which corresponds to no center-of-mass motion. Thus in the ground state, $\mathbf{k}_{\mathrm{CM}}=0$ and we have a pair of electrons with opposite momenta $(\mathbf{k},-\mathbf{k})$. The spin wavefunction of the system can be, for example, spin singlet ( $s$-wave with $\ell=0$ or $d$-wave with $\ell=2$ ) or spin triplet $(p$-wave with $\ell=1)$, however the $s$-wave singlet pairing state is energetically most favourable. BCS expected this result, namely that the lowest-energy state will occur when the pairs of electrons have opposite spin and zero relative momentum. Hence, pairs of the form $(\mathbf{k} \uparrow,-\mathbf{k} \downarrow)$ were considered forming an $s$-wave bound state. Today, such bound states of electrons are known as Cooper pairs. In fact the entire Fermi surface will become unstable towards the formation of Cooper pairs which then condense altogether to form a macroscopic coherent state.

We now outline the essential equations within the BCS theory of superconductivity following the notation of Schrieffer [58]. First, upon discovering that an attractive interaction will make the Fermi surface unstable to the creation of Cooper pairs, BCS became motivated to solve the "reduced Hamiltonian",

$$
\hat{H}_{\mathrm{red}}=2 \sum_{\mathbf{k}} \epsilon_{\mathbf{k}} \hat{b}_{\mathbf{k}}^{\dagger} \hat{b}_{\mathbf{k}}+\sum_{\mathbf{k} \mathbf{k}^{\prime}} V_{\mathbf{k}^{\prime} \mathbf{k}} \hat{b}_{\mathbf{k}^{\prime}}^{\dagger} \hat{b}_{\mathbf{k}}
$$

derived from the full Hamiltonian of the system,

$$
H=\sum_{\mathbf{k} \sigma} \epsilon_{\mathbf{k}} \hat{c}_{\mathbf{k} \sigma}^{\dagger} \hat{c}_{\mathbf{k} \sigma}+H_{\mathrm{C}}+H_{\mathrm{el}-\mathrm{ph}},
$$


where the first term in (2.2) represents the kinetic energy with dispersion $\epsilon_{\mathbf{k}}$, the Coulomb interaction is $H_{\mathrm{C}}$, and the electron-phonon interactions are represented by $H_{\mathrm{el}-\mathrm{ph}}$. The reduced Hamiltonian then contains the kinetic energy plus a term representing the effective electron-electron interactions via exchange of photons. Here $\hat{b}_{\mathbf{k}}^{\dagger}$ and $\hat{b}_{\mathbf{k}}$ create and annihilate a Cooper pair in state $(\mathbf{k} \uparrow,-\mathbf{k} \downarrow)$, respectively, and $V_{\mathbf{k}^{\prime} \mathbf{k}}$ models the weak electron-phonon interaction that scatters a Cooper pair from state $\mathbf{k}$ to state $\mathbf{k}^{\prime}$. In terms of electron annihilation operations, the Cooper pair annihilation operator is given by $\hat{b}_{\mathbf{k}}=\hat{c}_{-\mathbf{k} \downarrow} \hat{c}_{\mathbf{k} \uparrow}$. While the Cooper pair operators satisfy the following commutation relations,

$$
\begin{aligned}
& {\left[\hat{b}_{\mathbf{k}}, \hat{b}_{\mathbf{k}^{\prime}}^{\dagger}\right]=0 ; \quad \mathbf{k} \neq \mathbf{k}^{\prime},} \\
& {\left[\hat{b}_{\mathbf{k}}, \hat{b}_{\mathbf{k}^{\prime}}\right]=0=\left[\hat{b}_{\mathbf{k}}^{\dagger}, \hat{b}_{\mathbf{k}^{\prime}}^{\dagger}\right],}
\end{aligned}
$$

they are not bosonic as we have

$$
\left[\hat{b}_{\mathbf{k}}, \hat{b}_{\mathbf{k}}^{\dagger}\right]=1-\left(\hat{n}_{\mathbf{k} \uparrow}+\hat{n}_{-\mathbf{k} \downarrow}\right)
$$

where $\hat{n}_{\mathbf{k} \sigma}=\hat{c}_{\mathbf{k} \sigma}^{\dagger} \hat{c}_{\mathbf{k} \sigma}$ is the number operator for the electron in state $(\mathbf{k}, \sigma)$. In an attempt to solve the reduced Hamiltonian (2.1), Schrieffer then proposed that at zero temperature the ground-state wavefunction could be written in the form

$$
\left|\Psi_{0}\right\rangle=C \prod_{\mathbf{k}}\left(1+\alpha_{\mathbf{k}} \hat{b}_{\mathbf{k}}^{\dagger}\right)|0\rangle
$$

where $C$ is a normalization constant and the $\left\{\alpha_{\mathbf{k}}\right\}$ are the parameters for the variational principle to minimize the ground-state energy. Upon normalization, we have

$$
C=\frac{1}{1+\left|\alpha_{\mathbf{k}}\right|^{2}}
$$

and the ground state can be written as

$$
\left|\Psi_{0}\right\rangle=\prod_{\mathbf{k}}\left(u_{\mathbf{k}}+v_{\mathbf{k}} \hat{b}_{\mathbf{k}}^{\dagger}\right)|0\rangle
$$


where

$$
\begin{aligned}
& u_{\mathrm{k}}=\frac{1}{1+\left|\alpha_{\mathbf{k}}\right|^{2}}, \\
& v_{\mathbf{k}}=\frac{\alpha_{\mathbf{k}}}{1+\left|\alpha_{\mathbf{k}}\right|^{2}} .
\end{aligned}
$$

Lastly, the orthonormality requires that we have

$$
\left|u_{\mathbf{k}}\right|^{2}+\left|v_{\mathbf{k}}\right|^{2}=1
$$

Physically, $u_{\mathbf{k}}$ is the probability amplitude for the ground state not to contain a Cooper pair in state $\mathbf{k}$ while $v_{\mathbf{k}}$ is the probability for the ground state to contain a Cooper pair.

Using $\left\{u_{\mathbf{k}}\right\}$ and $\left\{v_{\mathbf{k}}\right\}$ as variational parameters, the ground state energy of the system can be minimized with the normalization condition $\left|u_{\mathbf{k}}\right|^{2}+\left|v_{\mathbf{k}}\right|^{2}=1$ and the requirement that the total number of electrons is conserved on average. Using the method of Lagrange multipliers, we have

$$
\delta E=\delta\left\langle\Psi_{0}\left|\hat{H}_{\text {red }}-\mu \hat{N}\right| \Psi_{0}\right\rangle=0
$$

where the chemical potential $\mu$ enters as the Lagrange multiplier and $\hat{N}$ counts the number of particles. By performing the minimization (2.12), we obtain two linear equations:

$$
\begin{aligned}
& \left(\epsilon_{\mathbf{k}}-\mu\right) u_{\mathbf{k}}+\Delta_{\mathbf{k}} v_{\mathbf{k}}=E_{\mathbf{k}} u_{\mathbf{k}}, \\
& \Delta_{\mathbf{k}}^{*} u_{\mathbf{k}}-\left(\epsilon_{\mathbf{k}}-\mu\right) v_{\mathbf{k}}=E_{\mathbf{k}} v_{\mathbf{k}} .
\end{aligned}
$$

Here we have introduced the order parameter $\Delta_{\mathbf{k}}$, which must be determined self-consistently:

$$
\Delta_{\mathbf{k}}=-\sum_{\mathbf{k}^{\prime}} V_{\mathbf{k k}^{\prime}} u_{\mathbf{k}^{\prime}} v_{\mathbf{k}^{\prime}}^{*}
$$

Rewriting (2.13) and (2.14) in matrix form, we obtain

$$
\left(\begin{array}{cc}
\epsilon_{\mathrm{k}}-\mu & \Delta_{\mathrm{k}} \\
\Delta_{\mathrm{k}}^{*} & -\left(\epsilon_{\mathrm{k}}-\mu\right)
\end{array}\right)\left(\begin{array}{c}
u_{\mathrm{k}} \\
v_{\mathrm{k}}
\end{array}\right)=E_{\mathrm{k}}\left(\begin{array}{c}
u_{\mathrm{k}} \\
v_{\mathrm{k}}
\end{array}\right)
$$


The eigenvalues $E_{\mathbf{k}}$ are then given by

$$
E_{\mathbf{k}}=\sqrt{\left(\epsilon_{\mathbf{k}}-\mu\right)^{2}+\left|\Delta_{\mathbf{k}}\right|^{2}}
$$

and one also finds that

$$
\begin{aligned}
& u_{\mathrm{k}}^{2}=\frac{1}{2}\left(1+\frac{\epsilon_{\mathrm{k}}-\mu}{E_{\mathrm{k}}}\right), \\
& v_{\mathbf{k}}^{2}=\frac{1}{2}\left(1-\frac{\epsilon_{\mathrm{k}}-\mu}{E_{\mathrm{k}}}\right),
\end{aligned}
$$

with

$$
u_{\mathrm{k}} v_{\mathrm{k}}^{*}=\frac{\Delta_{\mathrm{k}}}{2 E_{\mathrm{k}}}
$$

Substituting (2.20) into (2.15), we obtain the famous BCS gap equation

$$
\Delta_{\mathbf{k}}=-\sum_{\mathbf{k}^{\prime}} V_{\mathbf{k}^{\prime} \mathbf{k}} \frac{\Delta_{\mathbf{k}^{\prime}}}{2 E_{\mathbf{k}}^{\prime}}
$$

For $s$-wave superconductivity, BCS introduced a simplified form of the gap equation, neglecting anisotropy thereby making the order parameter and the potential momentum-independent, $V_{\mathbf{k}^{\prime} \mathbf{k}}=-V$. In this case the order parameter can be assumed to be real and we obtain

$$
\Delta=\sum_{\mathbf{k}^{\prime}} V \frac{\Delta}{2 E_{\mathbf{k}^{\prime}}}
$$

Canceling $\Delta$ and converting the sum over $\mathbf{k}^{\prime}$ to an integral within an energy shell of $\left|\epsilon_{\mathbf{k}}-\mu\right|<$ $\hbar \omega_{D}$ for a bulk system, we obtain

$$
1 \simeq \frac{V}{2} D\left(\epsilon_{F}\right) \int_{-\hbar \omega_{D}}^{\hbar \omega_{D}} \frac{d \epsilon}{\sqrt{\epsilon^{2}+\Delta^{2}}}
$$

where $D\left(\epsilon_{F}\right)$ is the density of states at the Fermi energy approximated to be a constant within the energy shell. Performing the integral, one finds

$$
\Delta=\frac{\hbar \omega_{D}}{\sinh \left(\frac{1}{V D\left(\epsilon_{F}\right)}\right)}
$$


In the so-called weak-coupling limit where $V D\left(\epsilon_{F}\right) \ll 1,(2.24)$ reduces to

$$
\Delta=2 \hbar \omega_{D} e^{\left[-1 / V D\left(\epsilon_{F}\right)\right]}
$$

This is the BCS value of the gap at zero temperature.

To find excited states, we seek a set of new operators which will diagonalize our reduced Hamiltionian. The required transformation is a canonical transformation known as the Bogoliubov-Valatin transformation defined by

$$
\begin{gathered}
\hat{\gamma}_{\mathbf{k} \uparrow}^{\dagger}=u_{\mathbf{k}} \hat{c}_{\mathbf{k} \uparrow}^{\dagger}-v_{\mathbf{k}} \hat{c}_{-\mathbf{k} \downarrow}, \\
\hat{\gamma}_{-\mathbf{k} \downarrow}=u_{\mathbf{k}} \hat{c}_{-\mathbf{k} \downarrow}+v_{\mathbf{k}} \hat{c}_{\mathbf{k} \uparrow}^{\dagger} .
\end{gathered}
$$

These operators $\hat{\gamma}_{\mathbf{k} \sigma}^{\dagger}$ and $\hat{\gamma}_{\mathbf{k} \sigma}$ create and annihilate, respectively, a quasiparticle in state $(\mathbf{k}, \sigma)$. Quasiparticles are single particle excitations in a superconductor and the previously mentioned normal state quasiparticles are now simply referred to as electrons by convention. We also see now that $u_{\mathbf{k}}$ and $v_{\mathbf{k}}$ are the particle- and hole-amplitudes, respectively, of a single-particle excitation. From the operators $\hat{\gamma}_{\mathbf{k} \sigma}^{\dagger}, \hat{\gamma}_{\mathbf{k} \sigma}$, we clearly see that a quasiparticle in a superconductor is neither an electron nor a hole, but rather a superposition of an electron and a hole. In terms of the statistics they obey, we find that the quasiparticle operators are fermionic and obey the following commutation relations:

$$
\begin{aligned}
& \left\{\hat{\gamma}_{\mathbf{k} \sigma}, \hat{\gamma}_{\mathbf{k}^{\prime} \sigma^{\prime}}^{\dagger}\right\}=\delta_{\mathbf{k k}^{\prime}} \delta_{\sigma \sigma^{\prime}}, \\
& \left\{\hat{\gamma}_{\mathbf{k} \sigma}^{\dagger}, \hat{\gamma}_{\mathbf{k}^{\prime} \sigma^{\prime}}^{\dagger}\right\}=0=\left\{\hat{\gamma}_{\mathbf{k} \sigma}, \hat{\gamma}_{\mathbf{k}^{\prime} \sigma^{\prime}}\right\} .
\end{aligned}
$$

In addition, if we allow these operators act on the BCS ground state, we find that the ground state actually corresponds to the vacuum state for quasiparticles:

$$
\begin{gathered}
\hat{\gamma}_{\mathbf{k} \uparrow}\left|\Psi_{0}\right\rangle=0 \\
\hat{\gamma}_{-\mathbf{k} \downarrow}\left|\Psi_{0}\right\rangle=0,
\end{gathered}
$$

while $\hat{\gamma}_{\mathbf{k} \sigma}^{\dagger}$ will then create a quasiparticle in the superconducting state with momentum $\mathbf{k}$ 
and $\operatorname{spin} \sigma$

$$
\begin{gathered}
\hat{\gamma}_{\mathbf{k} \uparrow}^{\dagger}\left|\Psi_{0}\right\rangle=\left|\Psi_{\mathbf{k} \uparrow}\right\rangle \\
\hat{\gamma}_{-\mathbf{k} \downarrow}^{\dagger}\left|\Psi_{0}\right\rangle=\left|\Psi_{-\mathbf{k} \downarrow}\right\rangle .
\end{gathered}
$$

In a normal metal, we have $\Delta=0$ and every state is either a particle state or a hole state with the energy required to add or remove an electron at momentum $\mathbf{k}$ being $\epsilon_{\mathbf{k}}$ and $-\epsilon_{\mathbf{k}}$, respectively. However, in the superconducting state we add and remove not electrons, but quasiparticles and the minimum energy required to create a quasiparticle is $\Delta$. When the number of particles in the system is conserved, the energy required for an excitation is $2 \Delta$ - the energy gap of the superconductor.

\subsection{BdG Theory and the Extended Hubbard Model}

Ever since its formulation in 1957, the BCS theory has been very successful in describing bulk, homogeneous superconductors. However, since the theory is formulated in momentum space, it cannot describe superconductivity in the presence of a surface or any kind of impurities as in these cases, momentum is not a good quantum number. Therefore we adopt a generalization of the BCS theory formulated in real space known as Bogoliubov-de Gennes (BdG) theory [59]. The BdG theory was formed through the work of P. de Gennes and N. Bogoliubov and is significantly more powerful than the BCS theory as any kind of inhomogeneity or magnetic field can be readily incorporated.

Applying the BdG theory to a minimal tight-binding model, we are able to describe superconductivity within a model now known as the extended (attractive) Hubbard model. The Hubbard model was initially proposed in 1963 by J. Hubbard [60] and is one of the simplest models for describing the interaction between electrons in a solid. Despite its simplicity, the Hubbard model is very effective in describing the behavior of many condensed matter systems and continues to be used today. The model begins with a lattice of ions on which electrons can move around, "hopping" from one lattice site to another. In the solid, the electrons mutually interact via a screened Coulomb interaction and this interaction will 
be largest for two electrons occupying the same lattice site. No more than two electrons may occupy the same site due to the Pauli exclusion principle if we are assuming the simplest case in which the site is an ion with a single valence orbital. Interactions in the Hubbard model are then modeled by a term which is zero if the site is empty or contains one electron, and has a value of $U$ if two electrons with opposite spin occupy the same site. The Hamiltonian for the so-called repulsive Hubbard model can then be written in its simplest form as

$$
H_{H}=\sum_{\langle i j\rangle \sigma} t_{i j} \hat{c}_{i \sigma}^{\dagger} \hat{c}_{j \sigma}+\sum_{i \sigma}\left(\epsilon_{i}-\mu\right) \hat{n}_{i \sigma}+\sum_{i} U_{i i} \hat{n}_{i \uparrow} \hat{n}_{i \downarrow}
$$

Here electrons are modelled to have the kinetic energy to hop from site $j$ to site $i$ in the lattice with probability amplitude $t_{i j}$. The hopping is represented by the destruction of an electron at site $j$ and the subsequent creation of an electron at site $i$. In addition, the notation $\langle i j\rangle$ in the sum represents a sum over all sites $i$ and $j$ which the electron may hop to from site $i$. The second term contains the chemical potential $\mu$ which controls the filling and the impurity potential at site $i$ is given by $\epsilon_{i}$. Finally, the last term is the interaction energy with $U_{i i}=+U$ representing the screened Coulomb interaction of the band. Most notably, the repulsive Hubbard model is used to describe Mott insulator behaviour of the parent compounds for high-temperature superconductors.

This Hamiltonian can be extended to study superconductivity by changing the repulsive potential $U_{i i}=+U$ to that of an attractive one, $U_{i i}=-U$ between electrons occupying the same site in order to model the attractive electron-phonon interactions. In addition, one can include longer-range attractive interactions between electrons occupying neighboring sites to model, for example, $d$-wave coupling. Such an extended Hubbard Hamiltonian can then be written as

$$
H=\sum_{\langle i j\rangle \sigma} t_{i j} \hat{c}_{i \sigma}^{\dagger} \hat{c}_{j \sigma}+\sum_{i \sigma}\left(\epsilon_{i}-\mu\right) \hat{n}_{i \sigma}+\sum_{i} U_{i i} \hat{n}_{i \uparrow} \hat{n}_{i \downarrow}+\frac{1}{2} \sum_{\langle i j\rangle} \sum_{\sigma \sigma^{\prime}} U_{i j} \hat{n}_{i \sigma} \hat{n}_{j \sigma^{\prime}}
$$

where $U_{i i}$ is now attractive and represents $s$-wave coupling, while $U_{i j}$ represents longer-range interactions.

While the extended Hubbard Hamiltonian gives us a starting point for our calculations, 
it is impossible to solve exactly due to the two-body interaction terms. In order to obtain solutions, we must introduce an effective Hamiltonian where the two-body interactions are approximated by a one-body interaction multiplied by a mean field which must be solved self-consistently. Performing this approximation, one obtains the effective Hamiltonian

$H_{\mathrm{eff}}=H_{0}+\sum_{i \sigma} V_{i i}^{(H)} \hat{n}_{i \sigma}+\frac{1}{2} \sum_{\langle i j\rangle \sigma} V_{i j}^{(H)} \hat{n}_{i \sigma}-\frac{1}{2} \sum_{\langle i j\rangle \sigma} V_{i j}^{(F)} \hat{c}_{i \sigma}^{\dagger} \hat{c}_{j \sigma}+\sum_{i} \Delta_{i i} \hat{c}_{i \uparrow}^{\dagger} \hat{c}_{i \downarrow}^{\dagger}+\frac{1}{2} \sum_{\langle i j\rangle} \Delta_{i j} \hat{c}_{i \uparrow}^{\dagger} \hat{c}_{j \downarrow}^{\dagger}+$ H.c.,

where $H_{0}$ is the single-particle Hamiltonian,

$$
H_{0}=\sum_{\langle i j\rangle \sigma} t_{i j} \hat{c}_{i \sigma}^{\dagger} \hat{c}_{j \sigma}+\sum_{i \sigma}\left(\epsilon_{i}-\mu\right) \hat{n}_{i \sigma}
$$

and H.c. stands for Hermitian conjugate. Here several mean fields have been introduced. First, $V_{i i}^{(H)}$ is the on-site Hartree potential which is the average attractive potential the electron feels by being at a particular site $i$ with another electron. In addition, $V_{i j}^{(H)}$ is the off-site Hartree potential which the electron feels from neighbouring electrons and $V_{i j}^{(F)}$ is the Fock potential due to exchange-correlation effects between electrons at neighbouring sites both via the longer-range interaction $U_{i j}$. Finally, $\Delta_{i i}$ and $\Delta_{i j}$ are the on- and off-site order parameters. We determine these mean fields by minimizing the free energies

$$
\begin{gathered}
F=\langle H\rangle-T S, \\
F_{1}=\left\langle H_{\mathrm{eff}}\right\rangle-T S,
\end{gathered}
$$

where $T$ is temperature and $S$ is entropy. Performing the minimizations and comparing terms in (2.38) and (2.39), one finds that the mean fields are given by

$$
\begin{aligned}
V_{i i}^{(H)} & =U_{i i}\left\langle\hat{c}_{i \sigma}^{\dagger} \hat{c}_{i \sigma}\right\rangle, \\
V_{i j}^{(H)} & =U_{i j}\left\langle\hat{c}_{j \sigma}^{\dagger} \hat{c}_{j \sigma}\right\rangle, \\
V_{i j}^{(F)} & =\frac{1}{2} U_{i j}\left[\left\langle\hat{c}_{j \sigma}^{\dagger} \hat{c}_{i \sigma}\right\rangle+\left\langle\hat{c}_{i \sigma}^{\dagger} \hat{c}_{j \sigma}\right\rangle\right],
\end{aligned}
$$




$$
\begin{aligned}
\Delta_{i i} & =U_{i i}\left\langle\hat{c}_{i \downarrow} \hat{c}_{i \uparrow}\right\rangle, \\
\Delta_{i j} & =\frac{1}{2} U_{i j}\left[\left\langle\hat{c}_{j \downarrow} \hat{c}_{i \uparrow}\right\rangle+\left\langle\hat{c}_{i \downarrow} \hat{c}_{j \uparrow}\right\rangle\right] .
\end{aligned}
$$

\subsubsection{The BdG Equations}

As the effective Hamiltonian (2.36) now contains only one-body interactions, we wish to diagonalize it in order to obtain its eigenstates and corresponding eigenvalues. To accomplish this, we perform the Bogoliubov-Valatin transformation introduced in Section 2.1,

$$
\begin{aligned}
& \hat{c}_{i \uparrow}=\sum_{n}\left(\hat{\gamma}_{n \uparrow} u_{n}(i)-\hat{\gamma}_{n \downarrow}^{\dagger} v_{n}^{*}(i)\right) \\
& \hat{c}_{i \downarrow}=\sum_{n}\left(\hat{\gamma}_{n \downarrow} u_{n}(i)+\hat{\gamma}_{n \uparrow}^{\dagger} v_{n}^{*}(i)\right) .
\end{aligned}
$$

As the quasiparticle operators are fermionic, their occupation probability is determined by the Fermi-Dirac distribution function:

$$
\begin{aligned}
& \left\langle\hat{\gamma}_{n \sigma}^{\dagger} \hat{\gamma}_{m \sigma^{\prime}}\right\rangle=\delta_{m n} \delta_{\sigma \sigma^{\prime}} f\left(\epsilon_{n}\right) \\
& \left\langle\hat{\gamma}_{n \sigma} \hat{\gamma}_{m \sigma^{\prime}}\right\rangle=0
\end{aligned}
$$

where $f\left(\epsilon_{n}\right)$ is the Fermi-Dirac distribution function at fundamental temperature $\tau$ and energy $\epsilon_{n}$,

$$
f\left(\epsilon_{n}\right)=\frac{1}{e^{\epsilon_{n} / \tau}+1}
$$

Applying the transformations (2.45) and (2.46) to our effective Hamiltonian (2.36), the Hamiltonian is diagonalized as

$$
H_{\mathrm{eff}}=E_{0}+\sum_{n \sigma} \epsilon_{n} \hat{\gamma}_{n \sigma}^{\dagger} \hat{\gamma}_{n \sigma}
$$

with $E_{0}$ representing the ground state energy of the system and $\epsilon_{n}$ being the energy of an excitation in state $n$.

By evaluating the four commutators, $\left[\hat{c}_{i \uparrow}, H_{\mathrm{eff}}\right],\left[\hat{c}_{i \downarrow}, H_{\mathrm{eff}}\right],\left[H_{\mathrm{eff}}, \hat{\gamma}_{n, \sigma}\right],\left[H_{\mathrm{eff}}, \hat{\gamma}_{n, \sigma}^{\dagger}\right]$, one arrives 
at the Bogoliubov de-Gennes (BdG) equations [59]. These are a very powerful set of equations which generalize the Hartree-Fock equations to the case of superconductivity. In matrix form, the $\mathrm{BdG}$ equations are given by

$$
H_{\mathrm{BdG}}\left(\begin{array}{c}
u_{n} \\
v_{n}
\end{array}\right)=\epsilon_{n}\left(\begin{array}{c}
u_{n} \\
v_{n}
\end{array}\right)
$$

where

$$
H_{\mathrm{BdG}}=\left(\begin{array}{cc}
\hat{T}+\hat{V}^{(H)}+\hat{V}^{(F)} & \hat{\Delta} \\
\hat{\Delta}^{*} & -\left(\hat{T}^{*}+\hat{V}^{(H)}+\hat{V}^{(F)}\right)
\end{array}\right) \text {. }
$$

Here $\hat{T}$ is the kinetic energy or "hopping" matrix, $\hat{V}^{(H)}$ and $\hat{V}^{(F)}$ are the Hartree and Fock potentials and $\hat{\Delta}$ is the order parameter. The BdG equations are Schroödinger-like equations for the particle- and hole-amplitudes of a quasiparticle, $u_{n}$ and $v_{n}$, respectively, forming a $2 N \times 2 N$ eigenvalue problem with energy eigenvalues $\left\{\epsilon_{n}\right\}$ for a lattice with $N$ sites. Moreover, the BdG Hamiltonian has an intrinsic particle-hole symmetry expressed as

$$
\Xi H_{\mathrm{BdG}} \Xi^{-1}=-H_{\mathrm{BdG}}^{*}
$$

where $\Xi=\tau_{x} K\left(\Xi^{2}=+1\right), \tau$ are the Pauli matrices in particle-hole space and $K$ is the complex conjugation operator. Particle-hole symmetry implies that there is a redundancy in the system as for every eigenstate $\Psi$ with eigenvalue $E$, its particle-hole conjugate $\Xi \Psi$ will also be an eigenstate with eigenvalue $-E$.

We are now in a position to express our mean fields, $\Delta, V^{(H)}$ and $V^{(F)}$ in terms of the particle and hole amplitudes of a quasiparticle. If we use transformations (2.45) and (2.46) to replace the electron operators with quasiparticle operators in equations (2.40) to (2.44) and then use the relations (2.47) and (2.48), we obtain

$$
\begin{aligned}
\Delta_{i j} & =-\frac{1}{2} U_{i j} \sum_{n}\left[u_{n}(i) v_{n}^{*}(j)+u_{n}(j) v_{n}^{*}(i)\right]\left(1-2 f_{n}\right), \\
V_{i j}^{(H)} & =U_{i j} \sum_{n}\left[\left|u_{n}(j)\right|^{2} f_{n}+\left|v_{n}(j)\right|^{2}\left(1-f_{n}\right)\right],
\end{aligned}
$$




$$
V_{i j}^{(F)}=\frac{1}{2} U_{i j} \sum_{n}\left[\left\{u_{n}^{*}(i) u_{n}(j)+u_{n}(i) u_{n}^{*}(j)\right\} f_{n}+\left\{v_{n}(i) v_{n}^{*}(j)+v_{n}^{*}(i) v_{n}(j)\right\}\left(1-f_{n}\right)\right] .
$$

Starting from an initial guess for the mean fields, traditionally one diagonalizes the BdG Hamiltonian (2.52) in order to obtain the eigenvalues and eigenvectors $\left\{\epsilon_{n}, u_{n}, v_{n}\right\}$ which are in turn used to recompute the mean fields using equations (2.54), (2.55) and (2.56). This process is then repeated until a desired convergence has been achieved. After the mean fields have converged, one can then calculate the average electron density at site $i$ from the particle and hole amplitudes,

$$
\left\langle n_{i}\right\rangle=2 \sum_{n}\left[\left|u_{n}(i)\right|^{2} f_{n}+\left|v_{n}(i)\right|^{2}\left(1-f_{n}\right)\right]
$$

Lastly, the local density of states (LDOS) after convergence is given by

$$
A_{i}(\omega)=\sum_{i}\left[\left|u_{n}(i)\right|^{2} \delta\left(\omega-\epsilon_{n}\right)+\left|v_{n}(i)\right|^{2} \delta\left(\omega+\epsilon_{n}\right)\right]
$$

As the LDOS can be directly measured via scanning tunneling spectroscopy (STS), it is a particularity useful tool for comparison with experimental data.

\subsection{Charge Density Waves}

The popularization of the study of charge density waves (CDW) - periodic modulations in the charge distribution in a material - began in 1974 with the discovery of CDW in transition metal dichalcogenides (TMDs) [61]. TMDs are layered crystals with an X-M-X structure where $\mathrm{X}$ are hexagonally packed chalcogen atoms and $\mathrm{M}$ is a transition metal. Some of these materials are particularly interesting in the context of superconductivity as they exhibit not only CDW, but also conventional low-temperature superconductivity and hence they motivated much research in the study of the relation between CDW and superconductivity. Another surprising property of TMDs is that some of these materials are actually known to become better conductors in the CDW state. However, despite the fact that charge density wave materials have been studied for almost four decades, the possible interplay of CDW and $\mathrm{SC}$ is still under intense debate $[51,52]$. Furthermore, surprisingly, the mechanism behind what is causing these charge modulations in CDW prototype materials has not been 
understood.

One possible mechanism is a concept known as Fermi-surface nesting originally studied by Peierls in the 1950s $[62,63]$. Nesting occurs when the Fermi-surface topology is such that certain sections of the Fermi surface are parallel to each other and hence coincide when shifted rigidly [64]. To see how nesting leads to CDW, consider the following 1D problem studied by Peierls: take a one-dimensional lattice of non-interacting particles with lattice spacing $a$ in a weak periodic potential $V$ with energy dispersion $E=k^{2}$ and assume the system is at half-filling so that there is one electron per site on average. Then, displace every second atom by a small amount $\delta$ thereby reducing the symmetry of the chain to that of one with spacing $2 a$. Ordinarily for such a system, the weak periodic potential will introduce energy gaps at $k= \pm \pi / a$. However, this doubling will cause the potential to acquire a component with wavenumber $\pi / a=2 k_{F}$ and will result in band gaps opening at $k= \pm \pi / 2 a$ causing the system to show insulating behaviour. All the states that are raised by the change will be vacant and all the states that are lowered will be occupied meaning there is an overall energy reduction in the system. Peierls also demonstrated that this energy reduction will be proportional to $V^{2} \log V$ for small $V$ while the elastic energy is proportional to $V^{2}$. When the displacement $\delta$ is small, $V$ will then be proportional to $\delta$ and hence the ground state will always correspond to a distortion in the lattice causing CDW. This became known as the Peierls transition.

By looking at the the electronic susceptibility for a one-dimensional chain of length $L$,

$$
\chi(q, \omega)=\lim _{\alpha \rightarrow 0^{+}} \frac{1}{L} \sum_{k} \frac{f(k+q)-f(k)}{\epsilon(k+q)-\epsilon(k)-\omega-i \alpha},
$$

we can see how nesting comes into play. Here $f(k)$ is the Fermi-Dirac distribution function, $L$ is the length of the chain, $q$ and $\omega$ are the space and time Fourier components of a timedependent external potential and $\alpha \rightarrow 0^{+}$. First, note that we are interested in CDW that are "frozen in" and so we consider only the static susceptibility at $\omega=0$. This implies that only the real part of $\chi$ will be relevant as $\operatorname{Im} \chi$ vanishes at $\omega=0$. The real part of the 
susceptibility is given by

$$
\operatorname{Re} \chi(q, 0)=\lim _{\alpha \rightarrow 0} \frac{1}{L} \sum_{k} \frac{(f(k+q)-f(k))(\epsilon(k+q)-\epsilon(k))}{(\epsilon(k+q)-\epsilon(k))^{2}+\alpha^{2}} .
$$

Hence the sum will diverge when $\epsilon(k+q)-\epsilon(k)=0$ as $\alpha \rightarrow 0^{+}$, or in other words, constant energy surfaces connected by a common wavevector $q$ will cause a divergence. This will occur when there is nesting within the Fermi surface and the vector $q$ that connects the large parallel portions of the Fermi surface is known as a nesting vector. For the 1D chain, the Fermi surface consists only of the two points $\pm k_{F}$ so the nesting is trivial. However, take for example, a 3D crystal where conduction occurs along $1 \mathrm{D}$ chains such as $\mathrm{NbSe}_{3}$ and $\mathrm{TaS}_{3}$. The Fermi surface will then consist of two parallel sheets $k_{\|}= \pm k_{F}$. These two sheets are said to exhibit "perfect nesting" as the nesting vector $q=2 k_{F}$ will match them onto one another identically and $\chi$ will diverge strongly. Note that dimensionality is very important with nesting as the nesting will become weaker with increasing dimension since there will be a large reduction of parallel portions in the Fermi surface.

Since CDW tends toward insulating behaviour, it would seem as though CDW and SC would be competing orders. Despite this, however, whether CDW competes or cooperates with superconductivity remains undetermined $[51,52,53,54]$. In order to explain why some materials such as $2 \mathrm{H}-\mathrm{NbSe}_{2}$ and $2 \mathrm{H}-\mathrm{TaSe}_{2}$ actually become better conductors in the CDW state, in 1975 Rice and Scott [65] proposed that CDW could be caused by singularities in the density of states called van Hove singularities (vHs). For a two-dimensional hexagonal lattice as relevant for TMDs, Rice and Scott showed that a divergence in the susceptibility $\chi(\mathbf{q}, \omega)$ would occur in $\mathbf{q}$ space localized around the value $\mathbf{q}=\mathbf{q}_{0}$, where $\mathbf{q}_{0}$ is a wave vector that connects two saddle points (van Hove singularities) on the Fermi surface. Then, when the system transitions to the CDW state driven by vHs, a CDW energy gap opens up at the locations of the vHs, effectively removing them from the Fermi surface. When there are impurities in the system (as there are in any real material), the conduction electrons on or very close to the Fermi surface are scattered by them. However, around the vHs the electrons are slow moving and may experience inelastic scattering thereby becoming bound to an impurity. The density of states is very high at a vHs and so there are many such electrons 
and hence the "removal" of the vHs from the Fermi surface can enhance the conductivity and explain why superconductivity can actually be enhanced by CDW.

The concept of vHs causing CDW has been largely ignored for many decades and it is only relatively recently through two contradictory ARPES experiments $[51,52]$ that this idea was again brought to attention to the research community. While the results obtained by Kiss et al. [51] are consistent with the theory of Rice and Scott, claiming that CDW boosts $\mathrm{SC}$, the results obtained by Borisenko et al. [52] are consistent with the conventional view that CDW arises as a consequence of nesting and suppresses SC. Furthermore, it has been shown by Sadowski, Tanaka and Nagai [66] that CDW can be caused by vHs that are well below the Fermi surface and hence are not a result of a divergence in the susceptibility as considered by Rice and Scott. As a result of the many conflicting viewpoints, there is still no consensus as to the mechanism behind CDW. Whether the cause is nesting or vHs, in addition to whether CDW and SC are competing or cooperating orders, remains a hot topic in condensed matter research. 


\section{Chapter 3}

\section{NumericAl Methods}

Traditionally in the study of superconducting systems, the Hamiltonian is directly diagonalized in order to obtain self-consistent solutions of the BdG equations. As system size increases, however, this method quickly becomes impractical, requiring high computational demand. As such, we implement a combination of two numerical techniques designed to efficiently determine the Green function and eigenpairs in condensed matter systems. In order to self-consistently determine the mean fields in our superconducting systems, the socalled Chebyshev polynomial expansion method [47] has been implemented, and to obtain the eigenpairs of the converged system, the recently developed Sakurai-Sugiura (SS) method [49] has also been put into practice. The key feature of both of these algorithms is that neither require any direct diagonalization of the Hamiltonian allowing for significant speedup over the conventional method. Armed with this combination, an efficient, fully parallelized mean-field scheme has been developed which will later be applied in the context of topological superconductivity.

\subsection{The Chebyshev Polynomial Expansion Method}

Due to the size and complexity of the two-dimensional BdG Hamiltonian, we implement a fast and manageable algorithm for calculating the mean fields of the system self-consistently and without any matrix diagonalization. To this end, we have found success with the method of Chebyshev polynomial expansion whose main idea is to expand the spectral density of the Green function, $\hat{G}(z)=(z-\hat{\mathcal{H}})^{-1}$, with a set of Chebyshev polynomials. Using the spectral density which is given by the difference between the retarded and advanced Green functions $\hat{d}(\omega) \equiv \hat{G}^{R}(\omega)-\hat{G}^{A}(\omega)$, an entire mean-field scheme can be developed as shown by Weiße 
et al. [47] and Nagai et al. [48]. Historically, the idea of expanding the Green function or the spectral density of the Green function with a set of orthonormal polynomials has been successfully used by a variety of groups studying condensed matter systems [67, 68] beginning with Kunishima, Itoh and Tanaka [69].

Following the formulation of Ref. [50], we express the spectral density as

$$
[\hat{d}(\omega)]_{\alpha \beta}=-2 \pi i \sum_{\gamma=1}^{2 N} U_{\alpha \gamma} U_{\beta \gamma}^{*} \delta\left(\omega-\epsilon_{\gamma}\right),
$$

where $U$ is a unitary matrix and $\left\{\epsilon_{\gamma}\right\}$ are the eigenvalues of the BdG Hamiltonian. Expressing the mean fields in terms of $\hat{d}(\omega)$, we obtain

$$
\begin{aligned}
& \left\langle c_{i}^{\dagger} c_{j}\right\rangle=-\frac{1}{2 \pi i} \int_{-\infty}^{\infty} d \omega f(\omega) \mathbf{e}(j)^{T} \hat{d}(\omega) \mathbf{e}(i), \\
& \left\langle c_{i} c_{j}\right\rangle=-\frac{1}{2 \pi i} \int_{-\infty}^{\infty} d \omega f(\omega) \mathbf{e}(j)^{T} \hat{d}(\omega) \mathbf{h}(i),
\end{aligned}
$$

where $\mathbf{e}(i)$ and $\mathbf{h}(i)$ are unit vectors

$$
[\mathbf{e}(i)]_{\gamma}=\delta_{i, \gamma}, \quad[\mathbf{h}(i)]_{\gamma}=\delta_{i+N, \gamma},
$$

and $f$ is the Fermi-Dirac distribution function.

To obtain our mean-field scheme, we now expand the spectral density in terms of Chebyshev polynomials. In principle, various other orthonormal polynomials could also be used to develop a mean-field scheme such as the Legendre polynomials. However, Chebyshev polynomials are in general considered to be the optimal orthonormal set to expand a smooth function [47]. In order to formulate the scheme used, first recall the following useful relations for general orthonormal polynomials $\phi_{n}(x)$ which are real functions of $x \in[-1,1]$ and weights $W(x)$ and $w_{n}$,

$$
\begin{aligned}
\delta\left(x-x^{\prime}\right) & =\sum_{n=0}^{\infty} \frac{W(x)}{w_{n}} \phi_{n}(x) \phi_{n}\left(x^{\prime}\right), \\
w_{n} \delta_{n, m} & =\int_{-1}^{1} \phi_{n}(x) \phi_{m}(x) W(x) d x .
\end{aligned}
$$


In general, orthogonal polynomials also satisfy the following recurrence relation,

$$
\phi_{n+1}(x)=\left(a_{n}+b_{n} x\right) \phi_{n}(x)-c_{n} \phi_{n-1}(x) .
$$

As $x \in[-1,1]$, we normalize the eigenvalue spectrum by defining a new matrix $\hat{\mathcal{K}}$ which is simply $\hat{\mathcal{H}}$ rescaled as

$$
\hat{\mathcal{K}}=\frac{\hat{\mathcal{H}}-b I}{a} \quad \xi_{\gamma}=\frac{\epsilon_{\gamma}-b}{a}
$$

where $a=\left(E_{\max }-E_{\min }\right) / 2$ and $b=\left(E_{\max }+E_{\min }\right) / 2$, and all eigenvalues $\epsilon_{\gamma}$ of $\mathcal{H}$ are within $\left[E_{\min }, E_{\max }\right]$.

After rescaling our Hamiltonian, we can also define the components of the polynomial $\phi_{n}$ as a function of the matrix $\hat{\mathcal{K}}$,

$$
\left[\phi_{n}(\hat{\mathcal{K}})\right]_{\alpha \beta}=\sum_{\gamma=1}^{2 N} U_{\alpha \gamma} U_{\beta \gamma}^{*} \phi_{n}\left(\xi_{\gamma}\right)
$$

where $\phi_{n}\left(\xi_{\gamma}\right)$ is well defined in the scaled energy range $[-1,1]$. Then we substitute (3.5) into our expression for $\hat{d}(\omega)$ and use (3.9) to obtain

$$
\begin{aligned}
{[\hat{d}(\omega)]_{\alpha \beta} } & =-2 \pi i \sum_{\gamma=1}^{2 N} U_{\alpha \gamma} U_{\beta \gamma}^{*} \sum_{n=0}^{\infty} \frac{W(\omega)}{w_{n}} \phi_{n}(\omega) \phi_{n}\left(\xi_{\gamma}\right) \\
& =\frac{-2 \pi i}{a} \sum_{n=0}^{\infty} \frac{W(\omega)}{w_{n}} \phi_{n}(\omega) \phi_{n}(\hat{\mathcal{K}})
\end{aligned}
$$

and hence

$$
\mathbf{p}^{T} \hat{d}(\omega) \mathbf{q}=-\frac{2 \pi i}{a} \sum_{n=0}^{\infty} \frac{W(\omega)}{w_{n}} \phi_{n}(\omega) \mathbf{p}^{T} \mathbf{q}_{\mathbf{n}}
$$

for arbitrary real vectors $\mathbf{p}, \mathbf{q}$ and we have defined $\mathbf{q}_{\mathbf{n}}=\phi_{n}(\hat{\mathcal{K}}) \mathbf{q}$. Since $\phi_{n}\left(\xi_{\gamma}\right)$ is well defined in $[-1,1]$, the integrals in $(3.2)$ and $(3.3)$ become bound by the energy range $[-1,1]$ with $\omega=a x+b, x \in[-1,1]$. Hence our mean fields become

$$
\left\langle c_{i}^{\dagger} c_{j}\right\rangle=\sum_{n=0}^{\infty} \mathbf{e}(j)^{T} \mathbf{e}_{n}(i) \frac{\mathcal{T}_{n}}{w_{n}}
$$




$$
\left\langle c_{i} c_{j}\right\rangle=\sum_{n=0}^{\infty} \mathbf{e}(j)^{T} \mathbf{h}_{n}(i) \frac{\mathcal{T}_{n}}{w_{n}},
$$

where the term $\mathcal{T}_{n}$ is an integral that must be determined numerically,

$$
\mathcal{T}_{n}=\int_{-1}^{1} d x f(a x+b) W(x) \phi_{n}(x)
$$

and $\mathbf{e}_{n}(i), \mathbf{h}_{n}(i)$ are defined as

$$
\mathbf{e}_{n}(i)=\phi_{n}(\hat{\mathcal{K}}) \mathbf{e}(i), \quad \mathbf{h}_{n}(i)=\phi_{n}(\hat{\mathcal{K}}) \mathbf{h}(i)
$$

For the Chebyshev polynomials, use

$$
\phi_{n}(x)=\cos [n \arccos (x)]
$$

with the weights,

$$
W(x)=\frac{1}{\sqrt{1-x^{2}}}, \quad w_{n}=\frac{\pi}{2}\left(1+\delta_{n, 0}\right) .
$$

In order to determine the vectors $\mathbf{e}_{n}(i)$ and $\mathbf{h}_{n}(i)$ we use the recurrence relation that generates the Chebyshev polynomials. Namely, we use (3.7) with $a_{n}=0, b_{n}=2$ and $c_{n}=1$,

$$
\mathbf{q}_{n+1}=2 \hat{\mathcal{K}} \mathbf{q}_{n}-\mathbf{q}_{n-1}
$$

with $\mathbf{q}_{0}=\mathbf{q}$ and $\mathbf{q}_{1}=\hat{\mathcal{K}} \mathbf{q}$. Lastly, in this formulation the local density of states at site $i$ for energy $\omega$ is given by

$$
\begin{aligned}
N(\omega, i) & =-\frac{1}{2 \pi i} \mathbf{e}(i)^{T} \hat{d}(\omega) \mathbf{e}(i) \\
& =\frac{1}{a} \sum_{n} \frac{W(\omega)}{w_{n}} \phi_{n}(\omega) \mathbf{e}(i)^{T} \mathbf{e}_{n}(i) .
\end{aligned}
$$

We have thus generated an entire mean-field scheme based on the expansion of $\hat{d}(\omega)$ with Chebyshev polynomials. Inputting scaling values $a$ and $b$ and the number of terms in the sums, $n$, we can use recurrence relation (3.18) to calculate the mean fields (3.12) and (3.13) until self-consistency has been achieved. As we do not perform any diagonalization of the 
Hamiltonian $\mathcal{H}$, the complexity is brought down to $\mathcal{O}\left(N^{2}\right)$ as opposed to $\mathcal{O}\left(N^{3}\right)$. Moreover, while it is very challenging to effectively parallelize direct diagonalization of the Hamiltonian (2.52), in the present method one can easily parallelize the calculation of (3.12) and (3.13) to obtain excellent efficiency.

Let us obtain optimal values of the energy scaling value $a$ and the number of terms in the sums $n$ for a $24 \times 24$ conventional $s$-wave system with $b=0, \mu=0, U=-1.5 t$, and periodic boundary conditions (PBC). Setting $n=1200$, Fig. 3.1 (a) demonstrates how the value of the order parameter using direct diagonalization compares to values obtained by the Chebyshev polynomial expansion, varying $a$. The results show that the selection of either $a=8$ or $a=10$ will provide the best accuracy. Upon choosing $a=10$, we converge the order parameter beginning at $n=500$ and then steadily increase the number of terms in the sum. This is illustrated in Fig. 3.1 (b) showing that as the number of terms in the sums increase, the results tend to approach the order parameter value obtained from direct diagonalization.

(a)

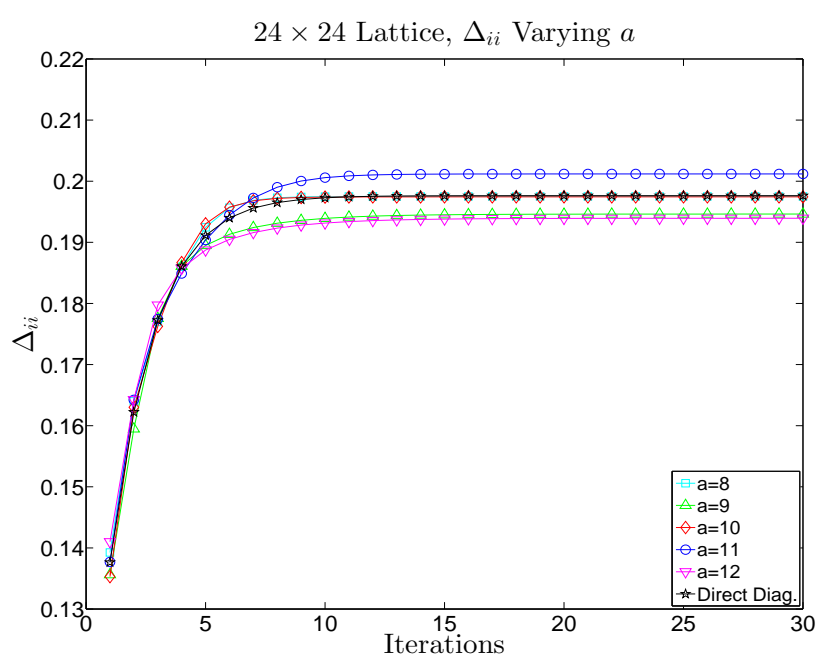

(b)

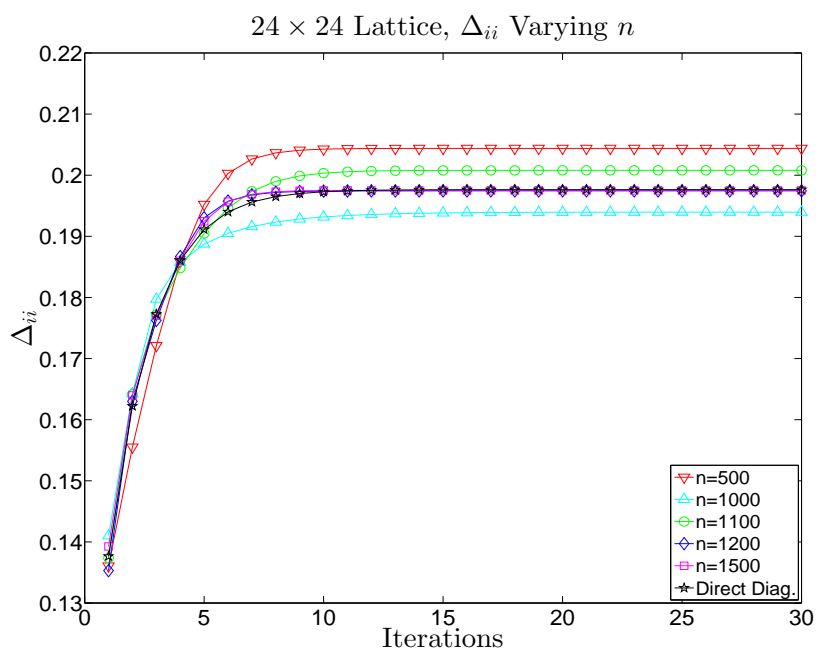

Figure 3.1: (a) Comparison of the order parameter obtained via direct diagonalization with the order parameter obtained varying $a$ with polynomial expansion $(n=1200, b=0)$. (b) Comparison of order parameters varying the number of terms in the sum, $n(a=10, b=0)$. The remaining parameters are $\mu=0$ and $U=-1.5 t$. 


\subsection{The Sakurai-Sugiura (SS) Method}

Now that we have an effective mean-field scheme and are able to perform self-consistent calculations, we wish to have a fast and efficient means to determine the eigenpairs of the system. The recently developed Sakurai-Sugiura method [49] provides us with this means by allowing the determination of eigenvalues and eigenvectors of the BdG Hamiltonian within a desired energy range without diagonalizing the entire matrix. The key to this method is the idea of being able to specify an energy range and calculate only those eigenvalues which lie in this range. Directly diagonalizing the Hamiltonian itself always provides us with the entire energy spectrum; however, one does not always need such the entire band structure and obtaining it every time can be a waste of computational resources. For example, in the study of topological superconductivity the interest usually lies in the detection of Majorana zero modes and the surrounding lowest-energy eigenvalues. For these reasons, the SS method is an excellent tool in the study of superconductivity as demonstrated by Nagai et al. $[50,70]$ and has proven to be extremely useful for the research presented in this thesis.

The SS method is a numerical solver for the generalized eigenvalue problem $A \mathbf{x}=\epsilon B \mathbf{x}$ with $A, B \in \mathbb{C}^{n_{s} \times n_{s}}$. The primary goal of this method is to reduce the size of the matrix $A$ as much as possible, reducing the eigenvalues to only those within the energy range specified. In order to accomplish this, we consider the $k$-th moment vector of the system

$$
\mathbf{s}_{k}=A^{k} P_{\Gamma}(A) \mathbf{v},
$$

where $k=0,1, \ldots, M-1, \mathbf{v} \in \mathbb{C}^{n_{s}}$ and $P_{\Gamma}(A)$ is the projection operator onto the eigenspace of $A$ :

$$
P_{\Gamma}(A)=\oint_{\Gamma} \frac{d z}{2 \pi i} \frac{1}{z I-A} .
$$

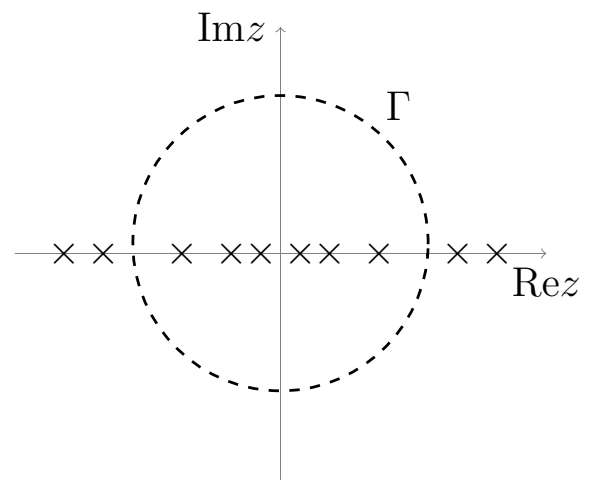

Figure 3.2: Schematic diagram of the contour on $\mathbb{C}$ with the eigenvalues marked on the real axis.

The method relies on the numerical evaluation of the contour integral representation of $\mathbf{s}_{k}$,

$$
\mathbf{s}_{k}=\oint_{\Gamma} \frac{d z}{2 \pi i} \frac{z^{k}}{z I-A} \mathbf{v},
$$


where the $M$ moment vectors of the system for an arbitrary vector $\mathbf{v}$ span a Krylov subspace of order $M$. Consider the contour $\Gamma$ on $\mathbb{C}$ in Fig. 3.2. This contour represents our energy range. Suppose that there are $m_{s}$ distinct eigenvalues inside this contour that we wish to obtain. We will now outline the process of obtaining these $m_{s}$ eigenvalues for a general Hamiltonian $\hat{\mathcal{H}}$. In our case, $\hat{\mathcal{H}}$ will be the BdG Hamiltonian (2.52).

(i) Specify a subspace size $M$, an initial guess $L \equiv L_{0}$ for the number of arbitrary vectors $\mathbf{v}$ required, a desired energy range which determines the contour $\Gamma$ (parameters $\alpha, \gamma$ and $\rho$ below), and the number of quadrature points $N_{q}$ for the contour integration.

(ii) Solve the system of equations $\left(z_{j} I-\hat{\mathcal{H}}\right) \hat{Y}_{j}=\hat{V}$ for $\hat{Y}_{j}, j=1, \ldots, N_{q}$, where $\hat{V}=$ $\left\{\mathbf{v}^{1}, \ldots, \mathbf{v}^{L_{0}}\right\}$ is called the source matrix and $z_{j}=\gamma+\rho\left(\cos \theta_{j}+i \alpha \sin \theta_{j}\right), \alpha \in(0,1]$ with $\theta_{j}=2 \pi / N_{q}(j-1 / 2)$.

(iii) Compute the moment matrix $\hat{S}_{k}=\frac{1}{N_{q}} \sum_{j=1}^{N_{q}} \rho \omega_{j} z_{j}^{k} \hat{Y}_{j}, k=0,1, \ldots, M-1$, where $\omega_{j}$ is the quadrature weight $\omega_{j}=\alpha \cos \theta_{j}+i \sin \theta_{j}$.

(iv) Using $\hat{S}_{0}$, we compute a "prior rank" $\tilde{m}_{s}$, which is estimated by $\tilde{m}_{s}=\frac{1}{L_{0}} \sum_{i=1}^{L_{0}}\left(\mathbf{v}^{i}\right)^{T} \mathbf{s}_{0}^{i}$. Here $\mathbf{s}_{0}^{i}$ is the $i$-th column of $S_{0}$. Using this prior rank, we obtain an improved value for $L$.

(v) Obtain a new $\hat{V}=\left\{\mathbf{v}^{1}, \ldots, \mathbf{v}^{L}\right\}$ and recompute $\hat{S}=\left\{\hat{S}_{0}, \ldots, \hat{S}_{M-1}\right\}$.

(vi) Perform singular-value decomposition (SVD) on $\hat{U} \hat{\Sigma} \hat{W}^{\dagger}=\hat{S}$ and find $m_{s}$ such that $\left|\sigma_{j}\right| /\left|\sigma_{1}\right|>\delta$ for singular values $\sigma_{j}, 1 \leq j \leq m_{s}$. Thus we have $m_{s}$ linearly independent vectors and as such $m_{s}$ eigenvalues in the contour.

(vii) Use the first $m_{s}$ columns of $\hat{U}$ to define a matrix $\tilde{Q}$.

(viii) Compute eigenvalues $\epsilon_{i}$ and eigenvectors $\mathbf{w}_{i}$ of the much smaller matrix $\tilde{H}=\tilde{Q}^{\dagger} \hat{\mathcal{H}} \tilde{Q}$.

(ix) Set $\mathbf{x}_{i}=\tilde{Q} \mathbf{w}_{i}$ and the desired eigenpairs $\left(\epsilon_{i}, \mathbf{x}_{i}\right)$ have been obtained. 
(a)

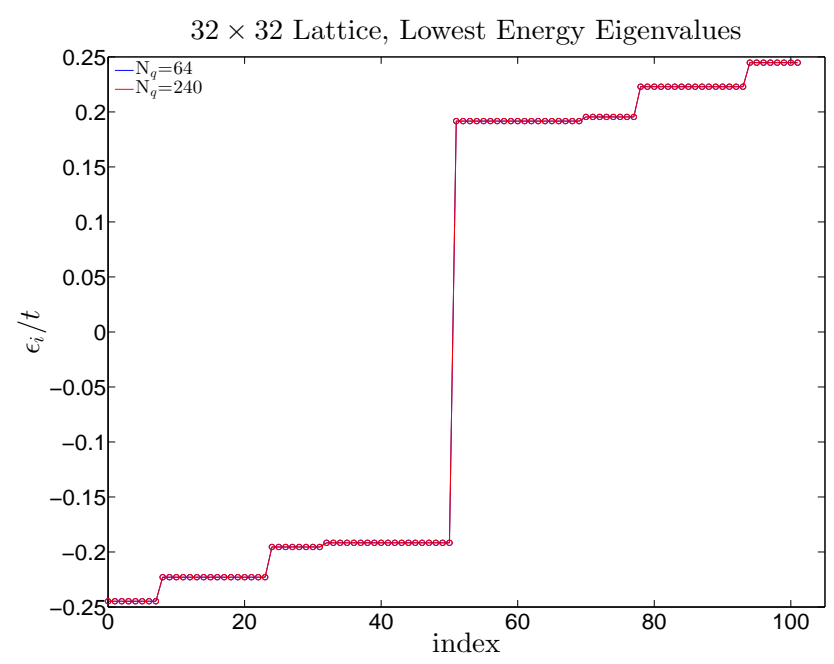

(b)

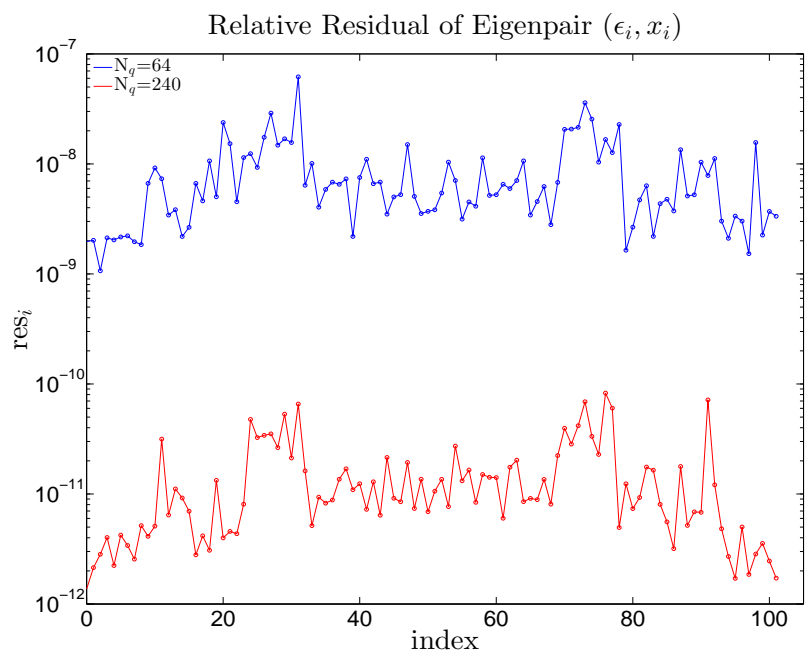

Figure 3.3: (a) Eigenvalues of the BdG Hamiltonian (2.52) with $\mu=0, U=-1.5 t$ and PBC, varying the number of quadrature points, $N_{q}$. The x-axis represents the index of the eigenvalues in ascending order. (b) Relative residual for each eigenpair varying $N_{q}$.

The accuracy of the SS method depends greatly on the size of the source $L$ and the number of quadrature points $N_{q}$. The simplest way to improve accuracy is to increase the number of quadrature points in the contour, although one has to be careful not to increase the number so much as to accumulate numerical errors. As a measure of the accuracy of an eigenpair $\left(\epsilon_{i}, \mathbf{x}_{i}\right)$, we compute the residual of the pair as

$$
\operatorname{res}_{i}=\frac{\left\|\hat{\mathcal{H}} \mathbf{x}_{\mathbf{i}}-\epsilon_{i} \mathbf{x}_{\mathbf{i}}\right\|}{\left\|\hat{\mathcal{H}} \mathbf{x}_{\mathbf{i}}\right\|+\left|\epsilon_{i}\left\|\mid \mathbf{x}_{\mathbf{i}}\right\|\right.},
$$

and discard any eigenpair with residual greater than 0.1 . Figure 3.3 shows a $32 \times 32$ swave system with $\mu=0, U=-1.5 t$ and $\mathrm{PBC}$ using $\rho=0.25$ which results in the contour $\Gamma\left(-0.25<\epsilon_{i}<0.25\right)$. The remaining parameters are $\alpha=0.5, L_{0}=10$ and $M=16$. While setting $N_{q}=64$ gives good precision of the numerical eigenvalues, $N_{q}=240$ shows significant improvement in the residuals.

Increasing the source size $L$ can also greatly improve the accuracy of the calculated eigenpairs. To demonstrate this, we compare the residuals of the 96 lowest energy eigenpairs for a $24 \times 24$ converged $s$-wave system with open boundary conditions (OBC), $\mu=0$ and $U=$ $-1.5 t$. The order parameter for this system is shown in Fig. 3.4 exhibiting Friedel oscillations 
along the diagonals due to quantum interference of the quasiparticle wavefunctions, and suppression along the edges from the loss of kinetic energy at the boundaries. We compute the eigenvalues of the system varying $L$ with $L_{0}=10, M=16$ and $N_{q}=64$. The initial calculation with $L=9$ produces eigenpairs with residuals that are quite large meaning the eigenpairs obtained are inaccurate, as can be seen in Fig. 3.5 (a). With increasing source size $L$, we see that the residuals of the lowest-energy eigenpairs become progressively smaller as $L$ increases. While increasing the source size is another simple way to obtain more accurate eigenvalues, each $\hat{S}_{k}$ is of size $n_{s} \times L$ and thus increasing $L$ can potentially lead to very time consuming computations. One way to overcome this is to refine the eigenpairs using a process called iterative refinement. In iterative refinement, one sets the source matrix $\hat{V}$ to be the zeroth-moment matrix,

$$
\hat{V}=\hat{S}_{0}
$$

and $r$ iterations of $P_{\Gamma}(\mathcal{H})$ on $\hat{V}$ are performed to obtain

$$
\hat{S}_{0}^{r}=P_{\Gamma}(\mathcal{H}) \hat{S}_{0}^{(r-1)}
$$

The $k$-th-moment vector is then constructed as

$$
\hat{S}_{k}^{(r)}=\mathcal{H}^{k} P_{\Gamma}(\mathcal{H}) \hat{S}_{0}^{(r-1)},
$$

which allows us to perform the singular value decomposition of $\hat{S}^{(r)}=\left\{\hat{S}_{0}^{r}, \ldots, \hat{S}_{M-1}^{(r)}\right\}$ and obtain refined eigenpairs. The results of such a computation are illustrated in Fig. 3.5 (b) where we have refined the eigenpairs obtained in Fig. 3.5 (a) with $L=9$. It was found that using $r=2$ in the refinement process resulted in a decrease of the residual average from $1.090 \times 10^{-2}$ to $7.005 \times 10^{-7}$, indicating that the refined eigenpairs are significantly more accurate. The downside to iterative refinement is the fact that a large portion of the SS method must be redone in order to obtain the refined eigenpairs. To avoid performing iterative refinement, the contour must be carefully selected to obtain eigenpairs with sufficiently small residuals yet not to generate an $L$ that is so large that the computation becomes too time-consuming. 


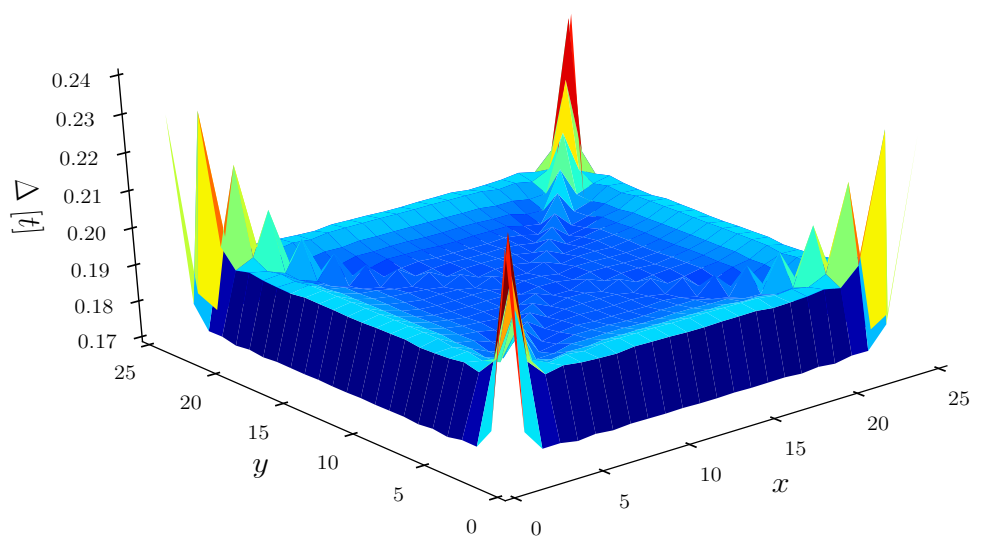

Figure 3.4: $O r d e r$ parameter for a $24 \times 24 s$-wave system with $\mu=0, U=-1.5 t, n=1500, a=10$ and $b=0$. The presence of open boundaries in both the $x$ - and $y$ - directions cause Friedel oscillations across the lattice.

(a)

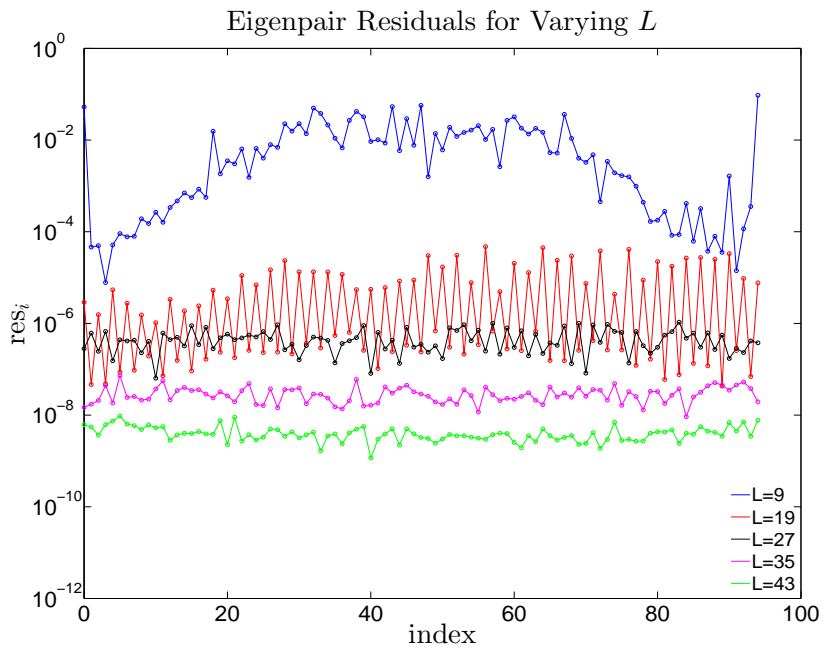

(b)

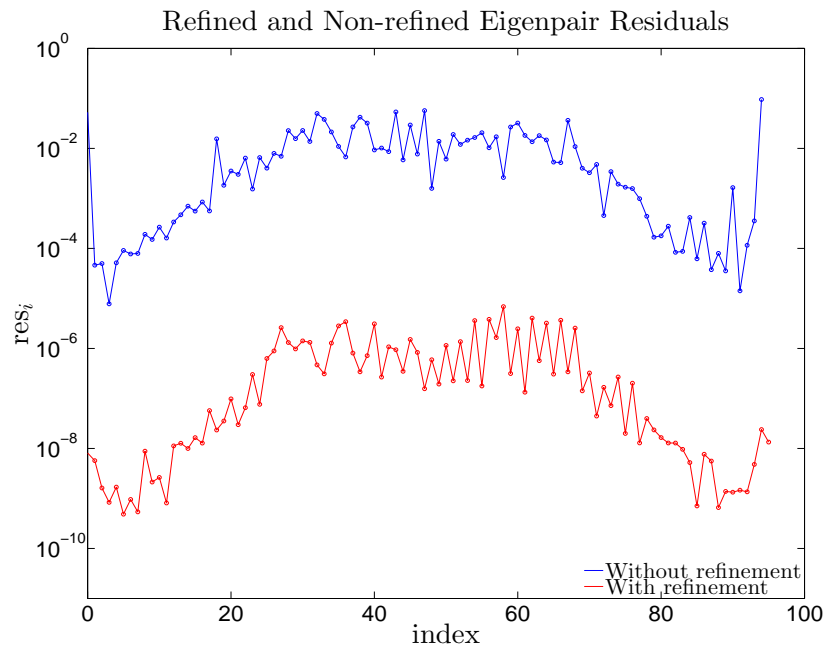

Figure 3.5: (a) Comparison of the residuals of the 96 lowest energy eigenpairs with increasing $L$ for a $24 \times 24$ s-wave system. (b) Comparison of the residuals obtained by the SS method with and without iterative refinement for the system shown in (a) with $L=9$.

As for efficiency, we remark that in the evaluation of $\hat{Y}_{j}$ in step (ii), one can separately solve each $\hat{Y}$ for $j=1, \ldots, N_{q}$ giving the SS method excellent parallel efficiency. In terms of complexity, the method is $\mathcal{O}\left(m_{s}^{2} N\right)$ where $N$ is the size of the $\mathrm{BdG}$ matrix. Writing $m_{s} \approx 1+a N$, we obtain a complexity of $\sim \mathcal{O}\left(N+2 a N^{2}+a^{2} N^{3}\right)$. To demonstrate this 
complexity, we refer to Fig. 3.6 where we show a log-log plot of elapsed time vs. lattice size $N_{x} \times N_{y}$ for a conventional $s$-wave superconductor again with $\mu=0, U=-1.5 t$ and PBC. Using 32 cores of the Grex cluster on Westgrid, calculations were performed on five different systems: $32 \times 32,48 \times 48,64 \times 64,80 \times 80$ and $96 \times 96$ for five different values of $\rho$ which determines the eigenvalue range. The remaining parameters are $\alpha=0.5, \gamma=0, L_{0}=10$, $M=16, N_{q}=64$. For small values of $\rho$, the linear term dominates the complexity and we have great parallel efficiency. As we increase the energy range $(\rho=0.25,0.5)$, the $N^{2}$ term mostly dominates, however we have considerable mixing in the complexity. Finally, as the energy range becomes relatively large $(\rho=1.0,1.5)$, the $N^{3}$ term greatly dominates bringing the complexity near that of directly diagonalizing the Hamiltonain as direct diagonalization is $\mathcal{O}\left(N^{3}\right)$.

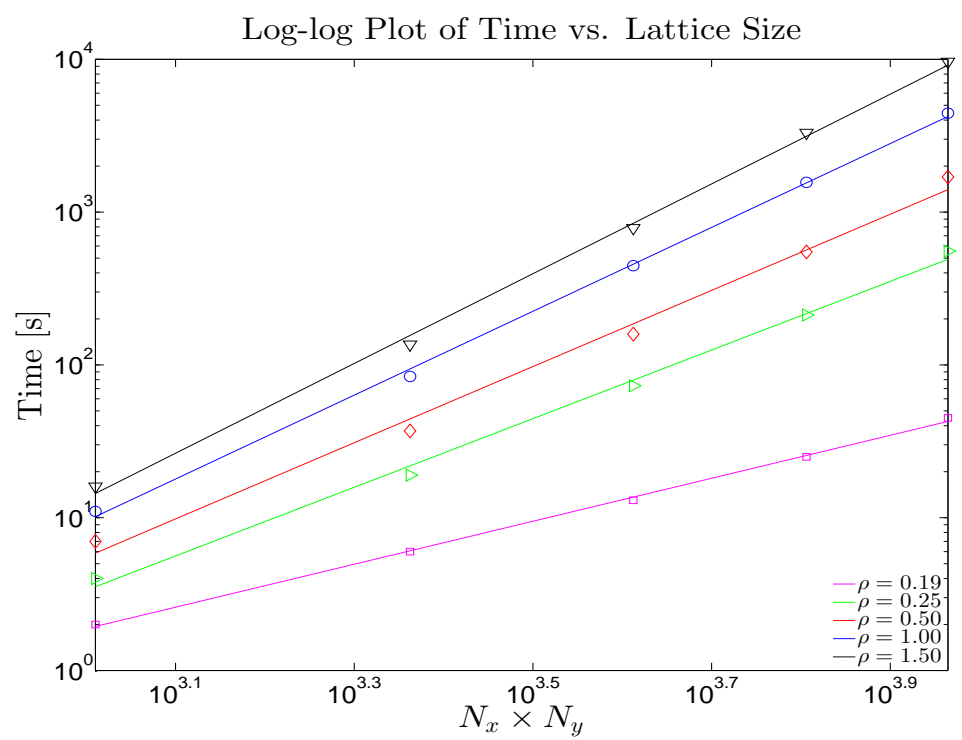

Figure 3.6: Lattice size dependence of the elapsed time for the SS method with varying energy range $\rho$ for an s-wave superconductor with $\mu=0$ and $U=-1.5 t$. 


\section{Chapter 4}

\section{Topological States of Matter}

The goal of this chapter is to provide sufficient theoretical background in the field of topological insulators and primarily topological superconductors in order to understand the results presented in later chapters. Every topological material can be characterized by two key features, namely, the existence of a bulk topological invariant and the presence of gapless edge states when the material is physically terminated. In this chapter, we discuss the concept of the TKNN number and the $Z_{2}$ index - the two most prominent topological invariants. We also examine the relation between these invariants and the gapless edge states that appear in topological systems. The chapter concludes with a discussion of the existence of Majorana fermions in topological superconductors and their application to topological quantum computation.

\subsection{The Quantum Hall System}

Discovered in 1980 by von Klitzing et al., the integer quantum Hall effect was historically the first example of a system that displayed topological behaviour. The effect arose when von Klitzing et al. measured the Hall resistance of a high-mobility two-dimensional electron gas in a semiconductor heterostructure in a high magnetic field at helium temperatures [71].

The integer quantum Hall effect describes the Hall resistivity $\rho_{x y}$ becoming quantized in integer multiples of $h / e^{2}$ and exhibiting plateaus as a function of the applied field strength $B$. Alternately, the Hall conductivity $\sigma_{x y}$ exhibits plateaus as a function of $1 / B$ at quantized values of

$$
\sigma_{x y}=\frac{e^{2}}{h} \nu
$$


where $\nu$ is an integer that corresponds to a certain number of filled Landau levels. Furthermore, whenever the Hall resistivity has a plateau, the conventional resistivity $\rho_{x x}$ is reduced drastically and approaches zero at $B$ within the plateau that corresponds to the number of completely filled Landau levels. This indicates that at such filling factors, the two-dimensional electron gas carries current with vanishing resistance.

One can historically consider the quantum Hall system to be the very first topological insulator - a material that has a bulk band gap but topologically protected conducting states at its surface or edge. Moreover, as was originally shown by Laughlin in 1981 [72], the integer quantum Hall effect in a system with open boundaries is indeed always accompanied by the presence of edge states which hinted at the topological nature of the system. The number of these edge states on one edge of the material must equal the Hall conductance in units of $e^{2} / h$ - a property that can be seen by using Laughlin's gauge argument [72]. Consider a two-dimensional electron gas in a magnetic field with periodic boundary conditions in the $y$ direction but with a finite width in the $x$ direction, like a ribbon forming a closed loop. We apply a magnetic field $B$ normal to the surface of the loop and our aim is to relate the Hall current $I_{y}$ to the Hall voltage $V_{x}$. Through the loop, we adiabatically insert a small amount of magnetic flux $\delta \Phi$ which creates a magnetic field $\delta H=\delta \Phi / A$ normal to the plane of the loop, where $A$ is the area enclosed by the loop. Hence the energy of the system increases by $\delta E=\left(I_{y} A / c\right) \delta H$ since the Hall current produces a magnetic moment of $I_{y} A / c$ and we can obtain the current as

$$
I_{y} / c=\frac{\delta E}{\delta \Phi} .
$$

We note that as Laughlin pointed out, the electrons in the Landau levels have extended wavefunctions and will be affected by the flux. On one edge, the electrons will have a higher electrostatic energy than those on the other edge due to the voltage applied across the loop. As the flux is slowly increased, the energy will rise as the electrons transfer from one edge to the other; however, the electrons only respond until the flux reaches exactly one flux quantum $h c / e$. By gauge invariance, when the flux reaches one flux quantum the system maps back into itself. If there are $n$ bands crossing the Fermi level, then the energy required during the flux insertion is given by the energy required to transfer $n$ electrons from one edge to the 
other:

$$
\delta E=n e V_{x} .
$$

Using $\delta \Phi=h c / e$, we then obtain

$$
\sigma_{x y}=\frac{I_{y}}{V_{x}}=n,
$$

in units of $e^{2} / h$.

Then in 1982, Thouless, Kohmoto, Nightingale, and den Nijs (TKNN) showed that another way to understand the integer quantum Hall effect is through the concept of topology and topological invariants. The concept of a material being "topological" lies in the topology of the system - fundamental properties of the material do not change when the material parameters are varied smoothly. In their seminal work, TKNN were able to show that in the quantum Hall system, $\mathbf{k}$-space is mapped onto a Hilbert space that has some non-trivial topology specified by an integer invariant [6] which is an invariant in the sense that its value does not change under smooth variations in the Hamiltonian. Moreover, the value of this integer or "topological" invariant $\nu$ is related to the Hall conductance $\sigma_{x y}$ such that $\sigma_{x y}$ equals $\nu$ times $e^{2} / h$. Using the Kubo formula [6],

$$
\sigma_{x y}=\frac{i e^{2}}{A \hbar} \sum_{\epsilon_{\alpha}<E_{F}} \sum_{\epsilon_{\beta}>E_{F}} \frac{\left(\partial \hat{\mathcal{H}} / \partial k_{1}\right)_{\alpha \beta}\left(\partial \hat{\mathcal{H}} / \partial k_{2}\right)_{\beta \alpha}-\left(\partial \hat{\mathcal{H}} / \partial k_{2}\right)_{\alpha \beta}\left(\partial \hat{\mathcal{H}} / \partial k_{1}\right)_{\beta \alpha}}{\left(\epsilon_{\alpha}-\epsilon_{\beta}\right)^{2}},
$$

where $A$ is the area of the system and $\epsilon_{\alpha}, \epsilon_{\beta}$ are the eigenvalues of the Hamiltonian $\hat{\mathcal{H}}$, TKNN directly showed this relation, breaking the first ground in the field of topological band theory. We now know this invariant $\nu$ to be the first Chern number or the TKNN number which is defined as the Berry phase of the Bloch wavefunction calculated around the boundary of the Brillouin zone. Piecing together the work of TKNN with the work of Laughlin, we also find that the TKNN number is equal to the number of conducting edge states that rise up on one edge. We will discuss the TKNN number in more mathematical detail in the next section. 


\subsection{The TKNN Number and $Z_{2}$ Index}

\subsubsection{The Berry Phase}

Perhaps the most important concept behind the field of topological band theory is the notion of the Berry phase. The Berry phase comes out rather naturally when studying the adiabatic theorem; however, it is interesting to note that this phase was overlooked by physicists for nearly 60 years. It was only in 1983 when Berry carefully studied a system under the adiabatic approximation that the Berry phase was discovered, earning its name. In the adiabatic approximation, the time scale over which a Hamiltonian is varied changes very slowly compared to other energy scales of the system. If the system starts at some initial time $t_{i}$ in an instantaneous eigenstate $\left|n\left(\mathbf{R}\left(t_{i}\right)\right)\right\rangle$, where $\mathbf{R}=\left(R_{1}(t), R_{2}(t), \ldots\right)$ is a set of timedependent parameters such as coordinates, then as time evolution takes place, the system will remain in this same eigenstate up to a phase factor known as the dynamical phase factor

$$
\theta_{D}(t)=\exp \left(-\frac{i}{\hbar} \int_{t_{i}}^{t_{f}} d t E_{n}(t)\right)
$$

However, in circumstances where the system adiabatically evolves along a closed path $C$ and at $t=t_{f}$, returns to its initial form at $t=t_{i}$, Berry discovered that the system returns to its initial state apart from a special phase factor that depends on the topology of the system,

$$
\theta(t)=\exp \left(i \gamma_{n}\right)
$$

where $\gamma_{n}$ is Berry's phase. We will now obtain a formula for $\gamma_{n}$ following the notation of Berry. The original derivation can be found in [73].

Consider a system with Hamiltonian $H(\mathbf{R})$ which depends on a time-dependent vector of

parameters as above, $\mathbf{R}=\left(R_{1}(t), R_{2}(t), \ldots\right)$ and denote its $n$-th eigenstate as $|n(\mathbf{R}(t))\rangle$. The Schrödinger equation for this system is given by

$$
H(\mathbf{R})|n(\mathbf{R})\rangle=E_{n}(\mathbf{R})|n(\mathbf{R})\rangle .
$$


We are considering adiabatic evolution of the system so that the parameters $\mathbf{R}(t)$ are varied very slowly starting from $\mathbf{R}(0)$ and going along a path $C$ in parameter space. We wish to examine the phase of the system $\theta(t)$ as the system evolves in time from its initial eigenstate $|n(\mathbf{R}(0))\rangle$. We can write the time evolution of this system as

$$
H(\mathbf{R}(t))|\psi(t)\rangle=i \hbar \frac{d}{d t}|\psi(t)\rangle
$$

where $|\psi(t)\rangle=e^{-i \theta(t)}|n(\mathbf{R})\rangle$. Performing the time derivative, we obtain

$$
E_{n}(\mathbf{R}(t))|n(\mathbf{R}(t))\rangle=\hbar\left(\frac{d}{d t} \theta(t)\right)|n(\mathbf{R}(t))\rangle+i \hbar \frac{d}{d t}|n(\mathbf{R}(t))\rangle
$$

Multiplying by $\langle n(\mathbf{R}(t))|$ and assuming the state is normalized, the differential equation becomes

$$
E_{n}(\mathbf{R}(t))-i \hbar\left\langle n(\mathbf{R}(t))\left|\frac{d}{d t}\right| n(\mathbf{R}(t))\right\rangle=\hbar\left(\frac{d}{d t} \theta(t)\right)
$$

Finally, we integrate and obtain for the phase $\theta(t)$,

$$
\theta(t)=\frac{1}{\hbar} \int_{0}^{t} E_{n}\left(\mathbf{R}\left(t^{\prime}\right)\right) d t^{\prime}-i \int_{0}^{t}\left\langle n\left(\mathbf{R}\left(t^{\prime}\right)\right)\left|\frac{d}{d t^{\prime}}\right| n\left(\mathbf{R}\left(t^{\prime}\right)\right)\right\rangle d t^{\prime}
$$

and we have

$$
|\psi(t)\rangle=\exp \left(-\frac{i}{\hbar} \int_{0}^{t} d t E_{n}(t)\right) \exp \left(i \gamma_{n}\right)|n(\mathbf{R}(t))\rangle
$$

We recognize the first exponential in (4.13) as the dynamical phase factor, and the second contains the Berry phase $\gamma_{n}$ given by

$$
\gamma_{n}=i \int_{0}^{t} d t^{\prime}\left\langle n\left(\mathbf{R}\left(t^{\prime}\right)\right)\left|\frac{d}{d t^{\prime}}\right| n\left(\mathbf{R}\left(t^{\prime}\right)\right)\right\rangle
$$

Note that the $\gamma_{n}$ is only defined up to a multiple of $2 \pi$. Suppose that the path $C$ is a closed 
curve in parameter space. Then we can write $\gamma_{n}$ as

$$
\begin{aligned}
\gamma_{n} & =i \int_{0}^{T} d t^{\prime} \frac{d \mathbf{R}}{d t^{\prime}}\left\langle n\left(\mathbf{R}\left(t^{\prime}\right)\right)\left|\nabla_{\mathbf{R}}\right| n\left(\mathbf{R}\left(t^{\prime}\right)\right)\right\rangle \\
& =i \oint_{\mathcal{C}} d \mathbf{R}\left\langle n(\mathbf{R})\left|\nabla_{\mathbf{R}}\right| n(\mathbf{R})\right\rangle \\
& =-\oint_{\mathcal{C}} d \mathbf{R} \cdot \mathbf{A}_{n}(\mathbf{R}),
\end{aligned}
$$

where $\mathbf{A}_{n}(\mathbf{R})$ is a vector potential known as the Berry vector potential or Berry connection,

$$
\mathbf{A}_{n}(\mathbf{R})=-i\left\langle n(\mathbf{R})\left|\nabla_{\mathbf{R}}\right| n(\mathbf{R})\right\rangle
$$

Naively when we examine (4.17), it seems as though the integral would always vanish as we have $\mathbf{R}(T)=\mathbf{R}(0)$, where $T$ is the time at which the closed path $C$ has been completed. However, this is not true as the integrand in (4.17) is not always an exact form, or in other words, not necessarily a total derivative [74]. It is interesting to note that before the work of Berry, it was thought that with an appropriate gauge transformation, one could simply cancel out $\gamma_{n}$. To see that this is incorrect, let us try to remove the Berry phase with a gauge transformation, $|n(\mathbf{R})\rangle \rightarrow e^{i \xi(\mathbf{R})}|n(\mathbf{R})\rangle$, where $\xi(\mathbf{R})$ is a smooth function and require that our eigenstates also be smooth and single-valued along $C$. The Berry vector potential then transforms as

$$
\mathbf{A}_{n}(\mathbf{R}) \rightarrow \mathbf{A}_{n}(\mathbf{R})-\frac{\partial}{\partial \mathbf{R}} \xi(\mathbf{R})
$$

and so the Berry phase becomes

$$
\gamma_{n} \rightarrow \gamma_{n}+\xi(\mathbf{R}(T))-\xi(\mathbf{R}(0))
$$

Then due to continuity, we must have $e^{i \xi(\mathbf{R}(0))}|n(\mathbf{R}(0))\rangle=e^{i \xi(\mathbf{R}(T))}|n(\mathbf{R}(T))\rangle=e^{i \xi(\mathbf{R}(T))}|n(\mathbf{R}(0))\rangle$ and thus $\xi(\mathbf{R}(T))-\xi(\mathbf{R}(0))=2 \pi m, m \in \mathbb{Z}$. And so we find that $\gamma_{n}$-which is only defined modulo $2 \pi$-is a gauge invariant quantity and cannot be removed.

Lastly we observe that the Berry phase is indeed a measurable quantity. In his original paper [73], Berry proposed a simple experiment for measuring $\gamma_{n}$. Consider a beam of particles in a magnetic field $\mathbf{B}$ in spin state $n$ with energy $E_{n}(B)$. Split this beam in two 
such that along the path of one beam, the magnetic field is constant. However along the path of the second beam, only the magnitude of the field is kept constant and its direction is allowed to vary slowly around a closed path $C$ forming a solid angle $\Omega$. The beams are then recombined and a detector measures the count rate as a function of $\Omega$. Because the energy $E_{n}$ depends only on the magnitude of the field, the dynamical phase of the two beams will be the same; however, the second beam in which the direction of the field varied will pick up a Berry phase. This simple experiment illustrates how the Berry phase is a geometrical property of the system depending on the path $C$ but not on the way the path is traversed. As such the Berry phase is also often referred to as the geometrical phase.

\subsubsection{The TKNN Number}

Closely related to the concept of the Berry phase is the TKNN number or first Chern number. As we saw in the previous section, the TKNN number is an integer that plays the role of the topological invariant in the quantum Hall system. In fact, the TKNN number plays the role of the topological invariant in all two-dimensional topological systems with timereversal symmetry (TRS) breaking, however the quantum Hall system is commonly used to illustrate the concept. In such systems, the TKNN integer will provide us with insight into the topology of the system by distinguishing between the trivial phase and the topological phase. A topological superconductor or insulator in the trivial phase will have a TKNN number of zero, while topological order in the system will be realized when the TKNN number is nonzero. This topological order signified by a nonzero topological invariant is encoded into the eigenstates of the system. In the topological phase, the eigenstates are rotated in Hilbert space and become twisted, meaning that one cannot continuously deform them into the eigenstates of a trivial insulator without making a "cut". Here this cut refers to closing the energy gap of the system. The TKNN number will not change under continuous deformations of the Hamiltonian that do not close the energy gap. In other words, when the system undergoes a transition from the trivial phase to the topological phase, the energy gap must close and the TKNN number changes from zero to nonzero. We also note that a system that preserves TRS cannot have a nonzero TKNN number since these systems cannot exhibit Hall effects. Mathematically, the TKNN number $\nu$ is given by the total Berry flux in 
the Brillouin zone

$$
\begin{aligned}
\nu & =\sum_{n} \frac{1}{2 \pi} \oint_{\partial B Z} d \mathbf{k} \cdot \mathbf{a}_{n}(\mathbf{k}) \\
& =-\frac{1}{2 \pi} \sum_{n} \gamma_{n}[\partial B Z],
\end{aligned}
$$

where $\mathbf{a}_{n}(\mathbf{k})=-i\left\langle u_{n \mathbf{k}}\left|\frac{\partial}{\partial \mathbf{k}}\right| u_{n \mathbf{k}}\right\rangle$ is the Berry connection for the Bloch states $\left|u_{n \mathbf{k}}\right\rangle$ and $\gamma_{n}$ is the Berry phase. A derivation of this result is presented in Appendix A. As we are constrained by the single-valuedness of the wavefunction, the change in phase after we complete a full path around the Brillouin zone boundary must be an integer multiple of $2 \pi$. Hence

$$
\gamma_{n}[\partial B Z]=2 \pi m, \quad m \in \mathbb{Z}
$$

and thus $\nu$ is necessarily an integer, as expected. And as discussed in the context of the quantum Hall effect, the TKNN number represents the quantized Hall conductivity for each band and we can write $\sigma_{x y}$ as

$$
\sigma_{x y}=\nu \frac{e^{2}}{h}
$$

In a periodic two-dimensional system, the topology of the Brillouin zone is that of a torus and as such, performing the integral in (4.21) will result in a TKNN number of zero since the torus has no boundary. Hence a nonzero TKNN number signifies the fact that the Berry vector potential has singularities at certain points in the Brillouin zone.

\subsubsection{The $Z_{2}$ Invariant}

In the late 1980s after TKNN's famous paper became well known, Haldane began to theoretically study the possibility of obtaining the quantum Hall effect in a two-dimensional graphene system in the paradoxical situation where the magnetic field is zero on average [75]. In his thought experiment, Haldane applied a periodic magnetic flux density normal to the plane of the lattice in order to break time-reversal symmetry, but kept the full translational symmetry so that every cell in the honeycomb lattice will have zero net flux. The effect of the field is to multiply the hopping elements by the Peierls phase factor $\exp [i(e / \hbar) \oint \mathbf{A} \cdot d \mathbf{r}][75]$ so that if 
we consider nearest-neighbour hopping only around a closed path, we obtain a phase factor of $\left.\exp \left[i(e / \hbar) \sum_{\text {cells }} \iint B d A\right)\right]=1$, since it is only possible to enclose an integer number of cells and $B=0$ within each of them. However, the key is that if we introduce next-nearestneighbour hopping and traverse a closed path, the phase accumulated can be nonzero as it is now possible to enclose not only integer multiples of hexagons but also portions of hexagons. Thus Haldane decided to introduce a next-nearest-neighbor hopping term of the form

$$
t^{\prime} \sum_{\langle\langle i, j\rangle\rangle} e^{i \alpha_{i j} \phi} c_{i}^{\dagger} c_{j}
$$

where $\sum_{\langle\langle i, j\rangle\rangle}$ represents the sum over all sites $i$ and $j$ which are next-nearest neighbours to $i, t^{\prime}$ is the next-nearest-neighbour hopping amplitude, $\phi$ is the phase acquired as we hop clockwise around the unit cell, and $\alpha_{i j}= \pm 1$ depending on whether the electron moves left $(-1)$ or right $(+1)$ going from its nearest neighbour to its second-nearest neighbour. He then wrote the full Hamiltonian of the system as

$$
H_{\text {Haldane }}=t \sum_{\langle i, j\rangle} c_{i}^{\dagger} c_{j}+t^{\prime} \sum_{\langle\langle i, j\rangle\rangle} e^{i \alpha_{i j} \phi} c_{i}^{\dagger} c_{j}+M \sum_{i} \epsilon_{i} c_{i}^{\dagger} c_{i}
$$

where $\epsilon_{i}= \pm 1$ depending on whether $i$ is on the $\mathrm{A}$ or B sublattice of the graphene and $M$ is an on-site inversion symmetry breaking term. This system is now known as Haldane's graphene model and is considered the first example of a topological insulator. One can compute the TKNN number which depends on the phase $\phi$ and $M$. Performing the computation, one obtains a phase diagram such as Fig. 4.1, adapted from Haldane's original work [75].

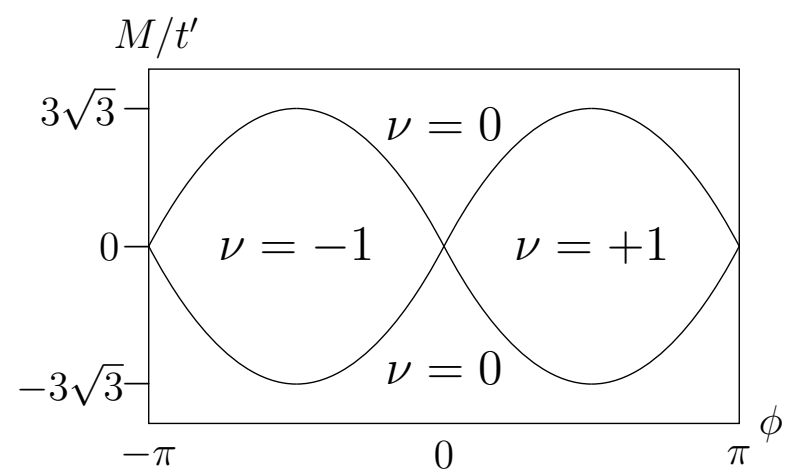

Figure 4.1: The dependence of the phases of the Haldane model on the parameters $M / t^{\prime}$ and $\phi$. The trivial phase is given by $\nu=0$ and the topological phase is given by $\nu= \pm 1$. Adapted from [75]. 
More than 15 years later, Kane and Mele proposed that if TRS remains intact, one can still obtain very interesting topological phases, albeit phases that are characterized by a different topological invariant [10]. The system that Kane and Mele examined was a timereversal-invariant quantum spin Hall state, obtained in the simplest way by doubling the Haldane model - one copy of the Hamiltonian for spin up and one for spin down. In this way we obtain two copies of the quantum Hall system which form a time-reversed pair that preserves the overall TRS, with the TRS given as $\Theta H(\mathbf{k}) \Theta^{\dagger}=H(-\mathbf{k})$. Here $\Theta=T K$ is the time-reversal operator and $K$ is a complex conjugation operator. In matrix form, we can write the Hamiltonian as

$$
H=\left(\begin{array}{cc}
H_{\uparrow} & 0 \\
0 & H_{\downarrow}
\end{array}\right)=\left(\begin{array}{cc}
H_{\text {Haldane }}(\phi=-\pi / 2) & 0 \\
0 & H_{\text {Haldane }}^{*}(\phi=\pi / 2)
\end{array}\right) .
$$

The spin-up Haldane model has a TKNN value of -1 and the spin-down model has a value of +1 [75], resulting in a TKNN number of $\nu=\nu_{\uparrow}+\nu_{\downarrow}=0$, as expected for a system with TRS. However, the difference $\nu_{\sigma}=\frac{1}{2}\left(\nu_{\uparrow}-\nu_{\downarrow}\right)$ is nonzero and defines a "spin TKNN number".

This is the simplest case in which $S_{z}$ is conserved; however more realistic systems can contain $S_{z}$-non-conserving terms such as spin-orbit coupling or disorder. To this end, Kane and Mele presented a more general quantum spin Hall system, namely a generalized version of Haldane's graphene model which included TR invariant spin-orbit coupling. Studying such a system, Kane and Mele were able to show that two distinct phases are exhibited within this model, a trivial insulator phase and a topological insulator phase characterized by an invariant which gives a classification based on parity [10]. Kane and Mele coined this new invariant the $Z_{2}$ invariant after the quotient group $\mathbb{Z} / 2 \mathbb{Z}$. The discovery of the $Z_{2}$ topology was a significant step towards the understanding of topological phases, since previous to their study, it was thought that TRS must be broken in order to obtain a topological phase. In order to compute this invariant, Kane and Mele introduced a formula that counts the number of pairs of zeros of the Pfaffian

$$
\left.P(\mathbf{k})=\operatorname{Pf}\left[\left\langle u_{i}(\mathbf{k})|\Theta| u_{j}(\mathbf{k})\right)\right\rangle\right]
$$


where $\left.\left.\mid u_{j}(\mathbf{k})\right)\right\rangle, j=1,2$ are the Bloch wavefunctions. As $\left.\left\langle u_{i}(\mathbf{k})|\Theta| u_{j}(\mathbf{k})\right)\right\rangle$ is a $2 \times 2$ antisymmetric matrix for this $2 \mathrm{D}$ case, the Pfaffian will simply pick out the $(1,2)$ element. The $Z_{2}$ index $\nu$ is then calculated by

$$
\nu=\frac{1}{2 \pi i} \oint_{C} d \mathbf{k} \cdot \nabla_{\mathbf{k}} \log (P(\mathbf{k})+i \delta)(\bmod 2)
$$

where $\delta \rightarrow 0^{+}$is a small convergence factor and $C$ is a loop enclosing only half the Brillouin zone. To understand this formula, it is conventional to introduce two subspaces: an even subspace for which $\Theta H(\mathbf{k}) \Theta^{\dagger}=H(-\mathbf{k})=H(\mathbf{k})$ and an odd subspace for which the space spanned by $\left.\left.\mid u_{j}(\mathbf{k})\right)\right\rangle$ is orthogonal to the space spanned by $\left.\left.\Theta \mid u_{j}(\mathbf{k})\right)\right\rangle$. In the even subspace, the Pfaffian has absolute value 1 and in the odd subspace the Pfaffian has a value of 0 . Moreover, in the odd subspace the zeros come in pairs since if there is a zero at $\mathbf{k}$, there will also be a zero at $\mathbf{- k}$. So when there are two pairs of zeros, say at $\left(\mathbf{k}_{1},-\mathbf{k}_{1}\right)$ and $\left(\mathbf{k}_{2},-\mathbf{k}_{2}\right)$, they can come together and annihilate when $\mathbf{k}_{1}=-\mathbf{k}_{2}$. However if there is only one pair of zeros at $\pm \mathbf{k}$, they cannot annihilate as that would only be possible at either the origin of the hexagonal Brillouin zone $\Gamma$, or the center point of an edge in the hexagon $M$, both of which belong to the even subspace. The number of pairs of zeros in the odd subspace $(\bmod 2)$ is a topological invariant known as the $Z_{2}$ index and is computed using (4.28). We integrate over only half the Brillouin zone so that $\mathbf{k}$ and $\mathbf{- k}$ are never both included so points instead of pairs are counted. If there are an odd number of pairs of zeros, the $Z_{2}$ index will be 1 and we have a topological insulator phase, and if there are an even number of pairs of zeros, they annihilate and the system is in the trivial phase. Note however that the stability of the topological phase depends critically on the presence of TRS. If TRS is broken, a pair of zeros at $\pm \mathbf{k}$ can in fact annihilate and hence we say that the topological phase is protected by time reversal symmetry.

\subsubsection{The Periodic Table of Topological Insulators and Supercon- ductors}

We discussed the $Z_{2}$ index only in the context of topological insulators as Kane and Mele formulated in their original paper. However, the $Z_{2}$ index is the topological invariant for 
many topological superconductors as well. All topological materials are characterized by a topological invariant (either the $Z_{2}$ index, the TKNN number, or a general integer winding number) which depends on the dimension of the system and the symmetries of the Hamiltonian. In total, there are four symmetry classes for topological superconductors and six for topological insulators, totaling ten symmetry classes. These symmetry classes are classified depending on the presence or absence of time-reversal symmetry $(\Theta)$, particle-hole symmetry $(\Xi)$ and chiral symmetry $(\Pi)$ which is given by the product of TRS and PHS, $\Pi=\Theta \Xi=0,1$. Chiral symmetry is also sometimes referred to as sublattice symmetry as it is the symmetry operation that flips the sign of the wavefunction on all sites of one of the two sublattices of the bipartite lattice. These invariants can be arranged into a "periodic table" of topological insulators and superconductors which was put together in the pioneering work of Schnyder et al. for dimensions $d=1,2,3$ in 2008 [76]. Today, topological materials are classified up to dimension $d=8$ and the invariants show a regular pattern that repeats when $d \rightarrow d+8[7]$. Here we summarize the table up to dimension $d=3$.

\begin{tabular}{l|cccccc}
\hline \hline & $\Theta$ & $\Xi$ & $\Pi$ & $d=1$ & $d=2$ & $d=3$ \\
\hline $\mathrm{A}$ & 0 & 0 & 0 & - & $\mathbb{Z}$ & - \\
$\mathrm{AI}$ & +1 & 0 & 0 & - & - & - \\
$\mathrm{AII}$ & -1 & 0 & 0 & - & $Z_{2}$ & $Z_{2}$ \\
$\mathrm{AIII}$ & 0 & 0 & 1 & $\mathbb{Z}$ & - & $\mathbb{Z}$ \\
$\mathrm{BDI}$ & +1 & +1 & 1 & $\mathbb{Z}$ & - & - \\
$\mathrm{CII}$ & -1 & -1 & 1 & $\mathbb{Z}$ & - & $Z_{2}$ \\
$\mathrm{D}$ & 0 & +1 & 0 & $Z_{2}$ & $\mathbb{Z}$ & - \\
$\mathrm{C}$ & 0 & -1 & 0 & - & $\mathbb{Z}$ & - \\
$\mathrm{DIII}$ & -1 & +1 & 1 & $Z_{2}$ & $Z_{2}$ & $\mathbb{Z}$ \\
$\mathrm{CI}$ & +1 & -1 & 1 & - & - & $\mathbb{Z}$ \\
\hline \hline
\end{tabular}

Table 4.1: The ten symmetry classes of topological insulators and superconductors and their corresponding invariant. $\Theta, \Xi$ and $\Pi$ correspond to time-reversal symmetry, particle-hole symmetry and chiral symmetry, respectively [76].

In Table 4.1, the absence of a particular symmetry is denoted by 0 . The values of \pm 1 for TRS and PHS are given by the square of the operator. In other words, if we have the presence of TRS and PHS, the value in the table corresponds to $\Theta^{2}= \pm 1$ and $\Xi^{2}= \pm 1$. The letters representing the symmetry classes originate from Altland and Zirnbauer and their work on classifying random matrices [77]. The classes $\{$ A, AI, AII $\}$ are the three "standard classes" 
where Hamiltonians of class A have no constraints other than being Hermitian, class AII imposes TRS and then class AI further imposes SU(2) spin rotational symmetry. The classes \{AIII, BDI, CII\} are known as the chiral classes where the Hamiltonians of class AIII have only chiral symmetry, CII adds TRS and then BDI has both TRS and SU(2) symmetries. Lastly $\{\mathrm{D}, \mathrm{C}, \mathrm{DIII}, \mathrm{CI}\}$ are known as the BdG (superconductor) classes. Class D has only PHS while class DIII has the addition of TRS but no SU(2) spin rotational symmetry. And finally, class C has SU(2) symmetry but no TRS, while CI contains both TRS and SU(2) spin rotational symmetry. An exhaustive description of each symmetry class can be found in [76]. In the present work, we focus exclusively on $2 \mathrm{D}$ topological superconductors of class $\mathrm{D}$ which has an integer classification given by the TKNN number.

\subsection{Edge States and Majorana Fermions}

In addition to being characterized by an integer topological invariant, the presence of gapless edge states is yet another key aspect of topological insulators and superconductors. These states appear when the topological insulator or superconductor is physically terminated and faces a material with a topological invariant different from the original material. As the topological invariant is always an integer, it is a discrete property of the system and cannot change unless the bulk gap is closed. For example, in order for the topology to change from nontrivial to trivial at the boundary between a topological superconductor and an ordinary material, there must be a gap-closing point at the boundary. Hence we arrive at the requirement for gapless edge states, called as such since they appear at the boundaries of topological insulators and superconductors and connect the valence band to the conduction band, crossing the Fermi level. For topological systems characterized by the TKNN number $\nu$, precisely $\nu$ edge states will appear on each edge when the boundary is shared with a trivial material, and $|\nu-\eta|$ edge states will appear when the boundary is between two topological materials with invariants $\nu$ and $\eta$. This principle is known as the bulk-boundary correspondence $[78,7]$.

In the quantum Hall state, these edge states are chiral in the sense that along an edge, there is propagation in only one direction. This property makes these edge states very robust 
to disorder as there can be no backscattering. By the bulk-boundary correspondence, the number of chiral edge states on one edge of the material must equal the TKNN invariant in the bulk. However, the situation is different in a system characterized by the $Z_{2}$ invariant, such as the quantum spin Hall system or a two-dimensional topological superconductor with TRS. In such a system, the edge states are no longer chiral, but helical meaning that each edge contains a pair of states that are time reversal conjugates of each other such that upspins propagate in one direction and down-spins propagate in the other as shown in Fig.

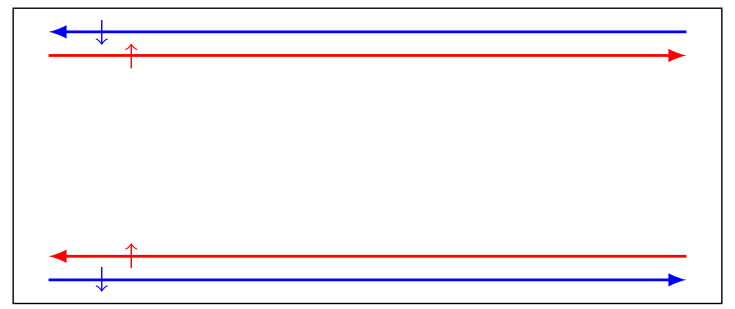

Figure 4.2: The spin-filtered helical edge states in the quantum spin Hall insulator. Here red represents spin up while blue represents spin down.
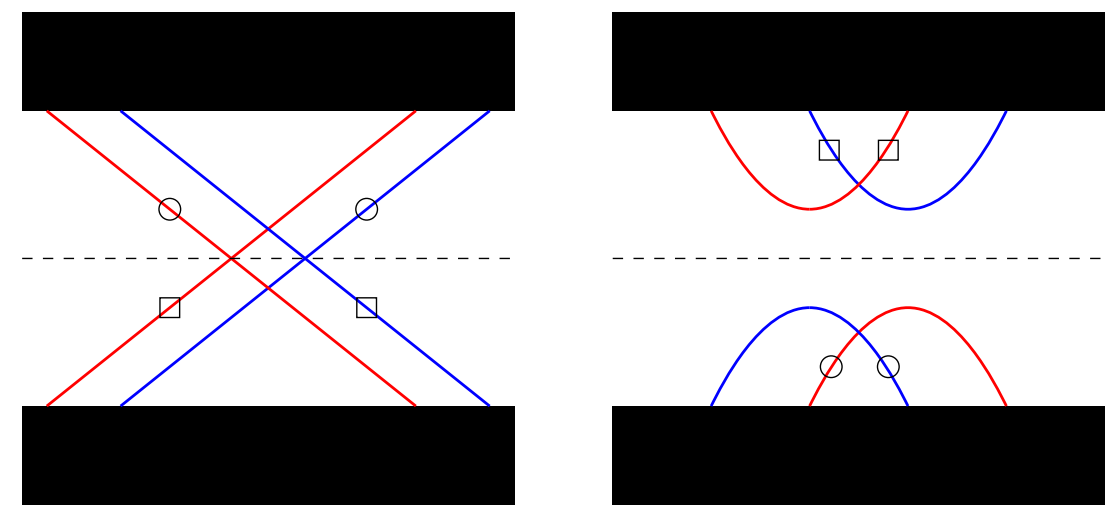

Figure 4.3: Edge states for the QSH system in the topological (left) and trivial (right) phases. The black blocks represent the bulk states whereas the red and blue lines represent the spin-up and spindown in-gap edge states, respectively. The circles and squares represent different edges of the material and the dotted line marks the Fermi energy. In the topological phase, there is an odd number of edge state pairs crossing the Fermi energy whereas in the trivial phase there are none. Note that while it is possible for the edge states to cross the Fermi level in the trivial phase if the Fermi energy were higher or lower, the difference is that the number of crossings would always be even.

In the topological phase, each edge will have at least one pair of such spin-filtered edge states which traverse the energy gap. Moreover, due to Kramers' theorem, the spin-up (down) states on each edge will cross at a time-reversal invariant point and this crossing will be protected by time-reversal symmetry $[8,10]$. In the trivial phase, these states do not 
traverse the gap as seen in Fig. 4.3 and there is no way to deform the trivial phase into the topological phase without closing the energy gap and making a "cut" in the eigenstates. Another way of computing the $Z_{2}$ index Kane and Mele showed, was to determine the number of edge-state pairs on each edge crossing the Fermi energy. If there are an odd number of edge state pairs on one edge, the system is in the topological phase and if there are an even number of such pairs, the system is in the trivial phase. The $Z_{2}$ index can then be summarized as

$$
\nu= \begin{cases}0, & \text { if an edge has an even number of edge-state pairs } \\ 1, & \text { if an edge has an odd number of edge-state pairs. }\end{cases}
$$

The robustness of these helical edge states has been well studied and it has been shown that only an odd number of pairs on an edge is robust against any time-reversal-symmetric perturbation. This is demonstrated clearly by $\mathrm{Xu}$ and Moore [79] who examined one edge of a 2-D fermionic system (a 1-D system) with $n$ pairs of edge modes, $n$ moving right and $n$ moving left with opposite spin under a perturbation $H^{\prime}$ that preserves TRS. At $t=0$ under a random potential, they questioned whether it was possible to scatter $n$ right movers back to $n$ left movers in the system. Let $|\psi\rangle$ be the final state of the $n$ left movers and assume that this is the time-reversal conjugate of the initial state of the right movers, $|\phi\rangle$. The matrix element for the perturbation to connect these states is [79]

$$
\begin{aligned}
\left\langle\psi\left|H^{\prime}\right| \phi\right\rangle & =\left\langle\Theta \phi\left|H^{\prime}\right| \phi\right\rangle=\left\langle\Theta H^{\prime} \phi \mid \Theta^{2} \phi\right\rangle=(-1)^{n}\left\langle\Theta H^{\prime} \phi \mid \phi\right\rangle \\
& =(-1)^{n}\left\langle H^{\prime} \Theta \phi \mid \phi\right\rangle=(-1)^{n}\left\langle\psi\left|H^{\prime}\right| \phi\right\rangle,
\end{aligned}
$$

where we have used the relation $\langle\Theta a \mid \Theta b\rangle=\langle b \mid a\rangle$. Hence, for $n$ odd, this scattering process is forbidden since the matrix element must be zero and so we conclude that an odd number of pairs on an edge will be robust to perturbations whereas an even number will not. As a final note, it is not true in general that trivial insulators or superconductors cannot have gapless edge states. The difference is that the edge states in a trivial insulator or superconductor are not robust to disorder and can be gapped out by perturbations.

In topological insulators, the electrons in these gapless edge states behave as massless Dirac fermions and the bulk electrons behave as massive Dirac fermions. What sets topo- 
logical superconductors apart from topological insulators, is the fact that topological superconductors have the remarkable property of hosting not massless Dirac fermions at their boundaries, but Majorana fermions. Majorana fermions were first proposed by Ettore Majorana in 1937 who discovered that if all $\gamma$ matrices are complex, one obtains a real solution to the Dirac equation such that a charge-neutral particle would be identical to its anti-particle. As a Majorana fermion is its own antiparticle, these particles satisfy the somewhat unusual anti-commutation relations:

$$
\left\{a_{j}^{\dagger}, a_{j^{\prime}}\right\}=\left\{a_{j}, a_{j^{\prime}}\right\}=\left\{a_{j}^{\dagger}, a_{j^{\prime}}^{\dagger}\right\}=2 \delta_{j j^{\prime}}
$$

where $a_{j}^{\dagger}$ and $a_{j}$ create and annihilate a Majorana fermion at lattice site $j$, respectively. In terms of these Majorana operators, the electron creation and annihilation operators $c_{j}^{\dagger}, c_{j}$ can be expressed as

$$
c_{j}=\frac{1}{2}\left(a_{2 j-1}+i a_{2 j}\right) \text { and } c_{j}^{\dagger}=\frac{1}{2}\left(a_{2 j-1}-i a_{2 j}\right) .
$$

We note that the electron creation and annihilation operators are comprised of two Majorana fermion operators. This is another reflection of the Majorana fermion being its own antiparticle - Majorana fermions have half the degrees of freedom than that of an electron [80].

Searching for Majorana fermions as quasiparticles in a superconductor is ideal as the charge-neutral quasiparticle is a superposition of a particle and a hole - the hole being the "antiparticle" of the electron. Due to the intrinsic particle-hole symmetry present in the system, every eigenstate of the BdG Hamiltonian with energy $E$ will have a particle-hole conjugate at energy $-E$. However, a Majorana fermion must be equal to its particle-hole conjugate at the same energy and hence in topological superconducting systems, Majorana fermions can only be realized at zero energy [7]. This is why Majorana fermions are commonly referred to as Majorana zero modes in the context of topological superconductivity. Majorana zero modes can be found localized at the location where the topological invariant changes, for example, at any boundaries present in the system. Hence in the bulk, we would observe no Majorana fermions.

The simplest toy model of a topological superconductor is the 1D p-wave wire of spinless 
fermions of class D first proposed by Kitaev in 2000 [30]. As we are dealing with spinless fermions, the orbital portion of the Cooper pair wavefunction is odd and hence a spin singletpairing potential is not possible to recover the overall oddness of the two-body wavefunction. Hence the only possibility for this model is p-wave superconductivity. As such, in the tightbinding limit the Hamiltonian for this system can be written as [30, 8]

$$
H_{1 \mathrm{D} \text { wire }}=\sum_{j}\left[-t\left(c_{j}^{\dagger} c_{j+1}+c_{j+1}^{\dagger} c_{j}\right)-\mu c_{j}^{\dagger} c_{j}+|\Delta|\left(c_{j+1}^{\dagger} c_{j}^{\dagger}+c_{j} c_{j+1}\right)\right]
$$

Or in terms of the Majorana creation and annihilation operators $a_{j}^{\dagger}, a_{j}$,

$$
H_{1 \mathrm{D} \text { wire }}=\frac{i}{2} \sum_{j}\left(-\mu a_{2 j-1} a_{2 j}+(t+|\Delta|) a_{2 j} a_{2 j+1}+(-t+|\Delta|) a_{2 j-1} a_{2 j+2}\right) .
$$

Using Hamiltonian (4.34), we present the two special cases found in [30, 8] that illustrate the difference between the trivial phase and the topological phase. Recall that it takes two Majorana fermions to comprise one electron, and hence two Majorana fermions will occupy each physical site within the 1D lattice.

1. $\mu<0,|\Delta|=t=0$

In this case, our Hamiltonian (4.34) reduces to

$$
H_{1 \mathrm{D} \text { wire }}=-\mu \frac{i}{2} \sum_{j} a_{2 j-1} a_{2 j}
$$

With open boundary conditions, we see that Majorana operator $a_{1}$ will be coupled to $a_{2}, a_{3}$ will be coupled to $a_{4}$, and so forth. Each pair of Majorana operators at each site are coupled to one another in the chain. Thus this limit represents the trivial phase. An illustration of this limit is shown in Fig. 4.4. 
2. $\mu=0,|\Delta|=t>0$

Here, our Hamiltonian reduces to

$$
H_{1 \mathrm{D} \text { wire }}=i t \sum_{j} a_{2 j} a_{2 j+1}
$$

Now, we see that with open boundary conditions, $a_{2}$ will be coupled to $a_{3}, a_{4}$ coupled to $a_{5}$ and so on, with the operators $a_{1}$ and $a_{L}$ not appearing in the Hamiltonian, where $L$ is the length of the chain. This leaves us with two dangling Majorana operators appearing at zero energy, each localized at opposite ends of the chain. If the chain is of infinite length, the coupling between these Majorana zero modes will be exactly zero. However, even if the chain is of finite length, Kitaev showed that the coupling will be exponentially small, vanishing as $\sim e^{-L}$. This implies that for a sufficiently long chain, the Majorana fermions will not be destroyed and will remain at zero energy unless the bulk gap closes. An illustration of this phase is also shown in Fig. 4.4.

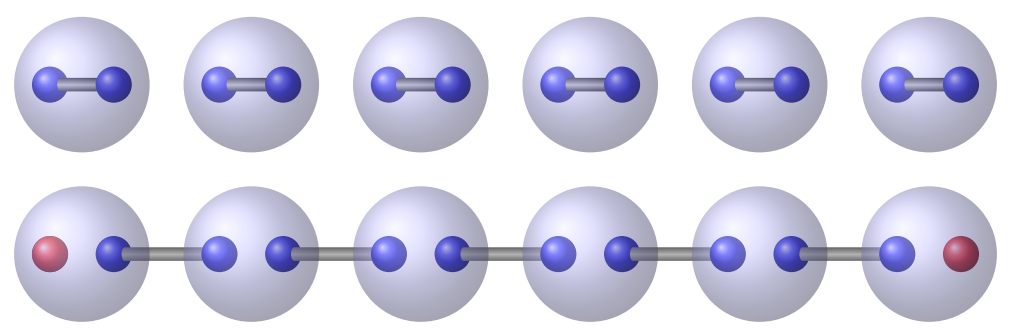

Figure 4.4: Illustration of the trivial (top) and topological (bottom) phases of the 1D p-wave wire with open boundary conditions. The small, dark blue spheres represent the Majorana operators making up each physical site. In the topological phase, we observe two dangling zero-energy Majorana modes localized at each end of the wire.

In two-dimensional topological superconducting systems, topological order can be further broken down into Abelian and non-Abelian topological order whereby the zero-energy modes will obey either Abelian or non-Abelian anyon statistics [55]. Anyons are exotic particles that exist only in two dimensions, and Abelian anyons obey so-called anyon statistics whereby the phase $\phi$ acquired under interchange of two particles ranges continuously between 0 and $\pi$ :

$$
\left|\psi_{1} \psi_{2}\right\rangle=e^{i \phi}\left|\psi_{2} \psi_{1}\right\rangle, \quad \phi \in(0, \pi)
$$

If the system has non-Abelian topological order, the zero-energy modes that appear are 
Majorana fermions that obey non-Abelian anyon statistics in which interchange between particles is not commutative. On the other hand, if the topological order is Abelian, the zero-energy modes are not Majorana fermions and instead obey the Abelian statistics given in (4.37).

Non-Abelian anyons are of particular interest due to their potential applications in the field of topological quantum computation [81]. Information in quantum computers is represented by quantum bits or "qubits" as opposed to a classical bit in a conventional computer. Whereas a classical bit takes on only two values, 0 or 1 , a qubit is a linear superposition of the states $|0\rangle$ and $|1\rangle$. This unique property gives qubits freedom that classical bits do not have, showing great potential for massively powerful computations. In a topological quantum computer, qubits are made up of non-Abelian anyons - two well-separated anyons to be precise. It is key that the anyons are well-separated so that a delocalized fermionic state is formed. In order to actually perform a computation, one gathers pairs of anyons and swaps them around one another in a determined sequence. The anyon's movement through time and space is known as its "worldline" and can be thought of as a thread which then braids around the worldlines of other anyons. The end result of the computation is then encoded in the braid that results when all the interchanges are complete [82]. The braid that is formed will then depend only on the topology of the overall braid and not on the intermediate details on how the braid was formed. Combined with the fact that qubits are nonlocal, braiding operations create a type of computation that is exceptionally robust to error as small disturbances in the paths of the anyons will not affect the computation [29]. Lastly, we see that it is important that the system be in the non-Abelian phase so that we obtain different results depending on the order in which the particles are swapped.

In this section we have discussed only Kitaev's simple spinless model, but in reality, the spin degree of freedom is a major obstacle for realizing Majorana fermions in topological superconducting systems. Recall for $s$-wave superconductivity, the quasiparticle operators from Chapter 2,

$$
\begin{gathered}
\hat{\gamma}_{\mathbf{k} \uparrow}^{\dagger}=u_{\mathbf{k}} \hat{c}_{\mathbf{k} \uparrow}^{\dagger}-v_{\mathbf{k}} \hat{c}_{-\mathbf{k} \downarrow} \\
\hat{\gamma}_{-\mathbf{k} \downarrow}=u_{\mathbf{k}} \hat{c}_{-\mathbf{k} \downarrow}+v_{\mathbf{k}} \hat{c}_{\mathbf{k} \uparrow}^{\dagger},
\end{gathered}
$$


where $u_{k}$ and $v_{k}$ are the particle and hole amplitudes of a quasiparticle, respectively. We see that due to the spin degree of freedom, there is no way that Majorana fermions can be realized within a conventional $s$-wave model as we cannot obtain $\gamma=\gamma^{\dagger}$. Hence in order to utilize an $s$-wave superconductor as a topological superconductor, we must have a way to "freeze out" the spin degree of freedom so that Cooper pairing can effectively occur between spinless particles. One way to do this is to make use of Rashba spin-orbit coupling as we will see in Chapter 5. 


\title{
CHAPTER 5
}

\section{Two Dimensional Topological Superconduc-}

\author{
TIVITY
}

\subsection{Topological S-Wave Superconductivity in Two Di- mensions}

The model adopted in this thesis is a two-dimensional (2D) $s$-wave topological superconductivity model proposed by Sato, Takahashi and Fujimoto [43] in the context of $s$-wave superfluids of ultracold fermionic atoms. Within this model, we perform microscopic mean-field calculations and self-consistently obtain converged solutions for both the superconducting order parameter and the Hartree potential. The tight-binding Hamiltonian for such an $s$-wave topological superconductor is given by [70]

$$
\mathcal{H}=\mathcal{H}_{K E}+\mathcal{H}_{S O}+\mathcal{H}_{s}
$$

where

$$
\begin{aligned}
\mathcal{H}_{K E} & =-t \sum_{\left\langle j, j^{\prime}\right\rangle} \sum_{\sigma} c_{j \sigma}^{\dagger} c_{j^{\prime} \sigma}+\sum_{j} \sum_{\sigma}\left(-\mu+\epsilon_{j}+V_{j j \bar{\sigma}}^{(H)}\right) c_{j \sigma}^{\dagger} c_{j \sigma}-h \sum_{j}\left(c_{j \downarrow}^{\dagger} c_{j \downarrow}-c_{j \uparrow}^{\dagger} c_{j \uparrow}\right)( \\
\mathcal{H}_{S O} & =-\frac{\alpha}{2}\left[\sum_{j}\left(c_{j-e_{x} \downarrow}^{\dagger} c_{j \uparrow}-c_{j+e_{x} \downarrow}^{\dagger} c_{j \uparrow}\right)+i\left(c_{j-e_{y} \downarrow}^{\dagger} c_{j \uparrow}-c_{j+e_{y} \downarrow}^{\dagger} c_{j \uparrow}\right)+\text { H.c. }\right] \\
\mathcal{H}_{s} & =\sum_{j}\left(\Delta_{i i} c_{j \uparrow}^{\dagger} c_{j \downarrow}^{\dagger}+\text { H.c. }\right) .
\end{aligned}
$$

Here $\sum_{\left\langle j, j^{\prime}\right\rangle}$ denotes a sum over nearest-neighbor sites, $t(>0)$ is the hopping matrix element, $\mu$ is the chemical potential, $\epsilon_{j}$ is a single-particle impurity potential at site $j, \alpha$ is the Rashba 
spin-orbit coupling constant, $\hat{e}_{x}$ and $\hat{e}_{y}$ are the unit vectors in the $x$ - and $y$-directions, $h$ is the magnitude of the Zeeman magnetic field, $\Delta$ is the superconducting order parameter, and H.c. stands for Hermitian conjugate. The electron creation and annihilation operators are denoted by $c_{j \sigma}^{\dagger}$ and $c_{j \sigma}$ at site $j$ with spin $\sigma$. The $s$-wave superconducting order parameter at site $i$ is given by

$$
\Delta_{i i}=U_{i i}\left\langle c_{i \downarrow} c_{i \uparrow}\right\rangle
$$

while the Hartree potential felt by the electrons with spin $\sigma$ and created by the electrons with opposite spin $\bar{\sigma}$ at site $i$ is given by

$$
V_{i i \bar{\sigma}}=U_{i i}\left\langle c_{i \bar{\sigma}}^{\dagger} c_{i \bar{\sigma}}\right\rangle
$$

As a minimal tight-binding model for studying superconductivity, we apply this model to a square lattice with nearest-neighbour hopping only. It is straightforward to extend the model to describe different band structures by including next-nearest-neighbour hopping, or by using different lattice structures such as a hexagonal lattice. Lastly, although Hamiltonian (5.1) is written in the notation of [70], the authors did not consider the effects of the Hartree potential within their study.

In order to examine the bulk spectrum of the system, we assume translational invariance and obtain the Hamiltonian in momentum space by Fourier transforming the real-space Hamiltonian (5.1) [55]:

$$
\begin{aligned}
\mathcal{H} & =\sum_{\mathbf{k}} \sum_{\sigma} \epsilon(\mathbf{k}) c_{\mathbf{k} \sigma}^{\dagger} c_{\mathbf{k} \sigma}-h \sum_{\mathbf{k}} \sum_{\sigma \sigma^{\prime}}\left(\sigma_{z}\right)_{\sigma \sigma^{\prime}} c_{\mathbf{k} \sigma}^{\dagger} c_{\mathbf{k} \sigma^{\prime}} \\
& +\alpha \sum_{\mathbf{k}} \sum_{\sigma, \sigma^{\prime}} \mathcal{L}(\mathbf{k}) \cdot \boldsymbol{\sigma}_{\sigma \sigma^{\prime}} c_{\mathbf{k} \sigma}^{\dagger} c_{\mathbf{k} \sigma^{\prime}} \\
& +\frac{1}{2}\left[\sum_{\mathbf{k}} \sum_{\sigma \sigma^{\prime}} \Delta_{\sigma \sigma^{\prime}}(\mathbf{k}) c_{\mathbf{k} \sigma}^{\dagger} c_{-\mathbf{k} \sigma^{\prime}}^{\dagger}+\text { H.c. }\right]
\end{aligned}
$$

where $\epsilon(\mathbf{k})=-2 t\left(\cos k_{x}+\cos k_{y}\right)-\mu$ is the energy dispersion, $\alpha \mathcal{L}(\mathbf{k})=\alpha\left(\sin k_{y},-\sin k_{x}\right)$ represents the Rashba spin-orbit coupling and $\sigma_{i}(i=x, y, z)$ are the Pauli matrices. Further 
rewriting (5.7) in matrix form, we obtain

$$
\mathcal{H}=\frac{1}{2} \sum_{\mathbf{k}}\left(c_{\mathbf{k} \uparrow}^{\dagger} c_{\mathbf{k} \downarrow}^{\dagger} c_{-\mathbf{k} \uparrow} c_{-\mathbf{k} \downarrow}\right) \mathcal{H}_{\mathrm{BdG}}(\mathbf{k})\left(\begin{array}{c}
c_{\mathbf{k} \uparrow} \\
c_{\mathbf{k} \downarrow} \\
c_{-\mathbf{k} \uparrow}^{\dagger} \\
c_{-\mathbf{k} \downarrow}^{\dagger}
\end{array}\right)
$$

where $\mathcal{H}_{\mathrm{BdG}}(\mathbf{k})$ is the BdG Hamiltonian,

$$
\mathcal{H}_{\mathrm{BdG}}(\mathbf{k})=\left(\begin{array}{cc}
\epsilon(\mathbf{k})-h \sigma_{z}+\alpha \mathcal{L}(\mathbf{k}) \cdot \boldsymbol{\sigma} & i \Delta(\mathbf{k}) \sigma_{y} \\
-i \Delta(\mathbf{k})^{*} \sigma_{y} & -\epsilon(\mathbf{k})+h \sigma_{z}+\alpha \mathcal{L}(\mathbf{k}) \cdot \boldsymbol{\sigma}
\end{array}\right)
$$

with $\mathcal{L}(\mathbf{k}) \cdot \boldsymbol{\sigma}=\sigma_{x} \sin k_{y}-\sigma_{y} \sin k_{x}$. Upon diagonalizing the Hamiltonian (5.9), we then obtain the bulk spectrum,

$$
E_{ \pm}(\mathbf{k})=\sqrt{\epsilon(\mathbf{k})^{2}+\alpha^{2}|\mathcal{L}(\mathbf{k})|^{2}+h^{2}+|\Delta(\mathbf{k})|^{2} \pm 2 \xi(\mathbf{k})}
$$

where $\xi(\mathbf{k})=\sqrt{\epsilon(\mathbf{k})^{2} \alpha^{2}|\mathcal{L}(\mathbf{k})|^{2}+\left(\epsilon(\mathbf{k})^{2}+|\Delta(\mathbf{k})|^{2}\right) h^{2}}$. To see the effects of the spin-orbit coupling and the Zeeman field, we plot the band energy $\epsilon_{ \pm}(\mathbf{k})=\epsilon(\mathbf{k}) \pm \sqrt{\alpha^{2}\left(\sin ^{2} k_{x}+\sin ^{2} k_{y}\right)+h^{2}}$ as a function of $k_{x}$ with $k_{y}=0$ in Fig. 5.1. When both $h=0$ and $\alpha=0$, the two energy bands are degenerate, as shown in Fig. 5.1 (a). The two energy bands are then split when we when we introduce the Rashba term with $h=0$ as shown in Fig. 5.1 (b), intersecting around the original band minimum. Until this point, time-reversal symmetry is still preserved. In order to break time-reversal symmetry we introduce a Zeeman magnetic field and for small Zeeman fields as in Fig. 5.1 (c) with $h=0.2 t$, a small gap opens at $k_{x}=0$ and $k_{x}= \pm \pi$. The gap increases in size as the Zeeman field increases in magnitude as shown in Fig. 5.1 (d) with $h=1.0 t$. Each energy band contains both spin-up and spin-down components, however as the Zeeman field continues to increase, the spin becomes increasingly aligned to the direction of the field and tends to break up spin-singlet Cooper pairs in an effect known as the Pauli depairing effect. Despite this effect, a large Zeeman field is desirable in order to push the two Fermi surfaces far apart, creating a large region in which to place the chemical potential so that one Fermi surface is eliminated. In this way, we have effectively created 
spinless superconductivity in that the spin direction is specified by $\mathbf{k}$, effectively removing the spin degree of freedom. It is through this type of superconductivity that we will observe the appearance of Majorana fermions.

(a)

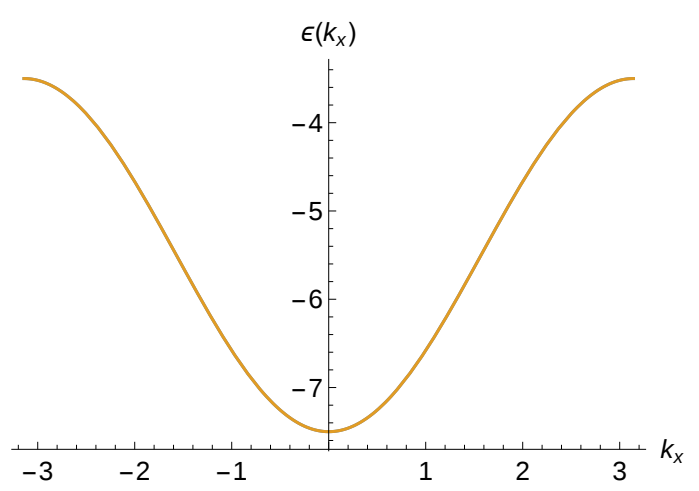

(c)

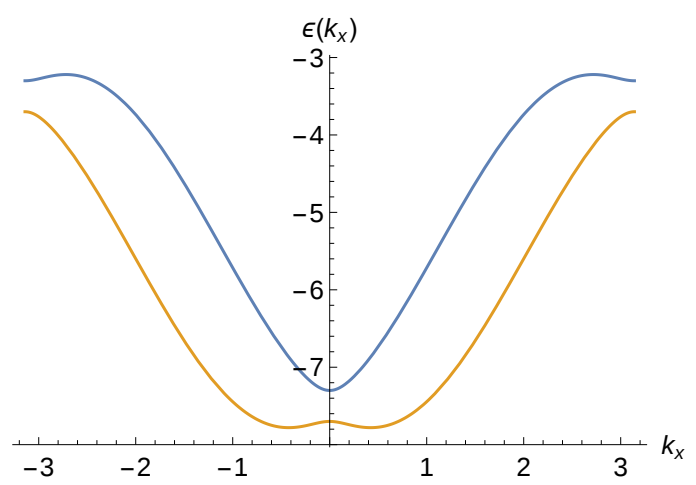

(b)

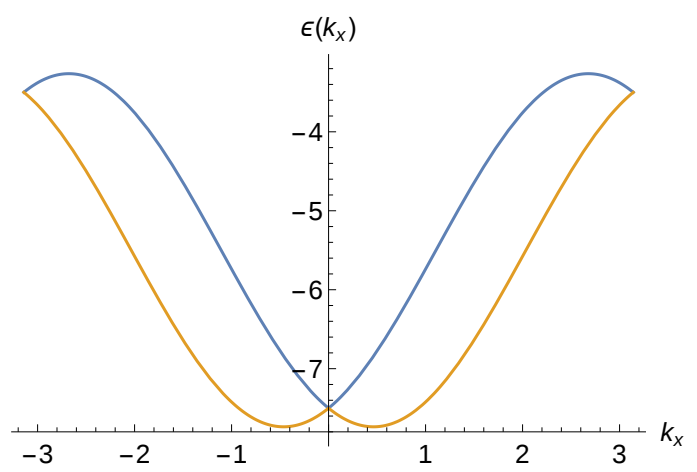

(d)

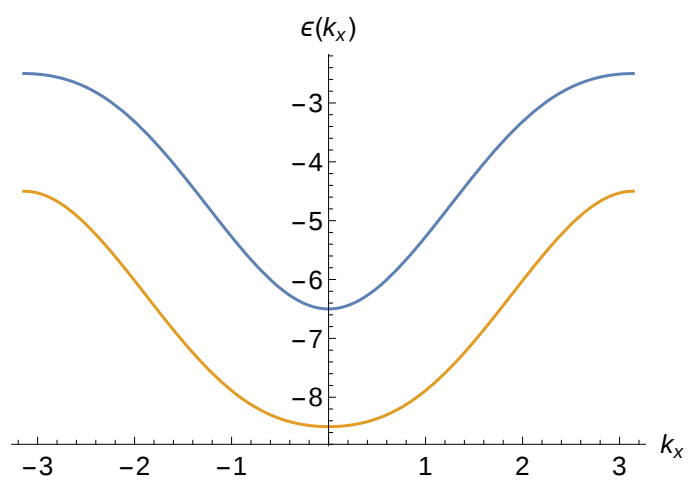

Figure 5.1: Energy bands $\epsilon_{+}$(blue) and $\epsilon_{-}$(orange) as a function of momentum $k_{x}$ with $\mu=3.5 t$ and $\Delta=0.5 t$. (a) The Zeeman field $h=0$ and the Rashba spin-orbit coupling $\alpha=0$. The two energy bands are degenerate. (b) $h=0$ and $\alpha=1.0 t$. The spin-orbit coupling splits the energy bands depending on their spin polarization. (c) $h=0.2 t$ and $\alpha=1.0 t$. The weak Zeeman field breaks TRS and opens a small gap at $k_{x}=0 .(\mathrm{d}) h=1.0 t$ and $\alpha=1.0 t$. The stronger Zeeman field opens a large gap at $k_{x}=0$.

The topological order within our system can be categorized into Abelian and non-Abelian topological order - the former being the case in which the zero-energy modes that appear in the system are Dirac fermions, like the zero-energy modes in a topological insulator. In the latter case, the zero-energy modes are Majorana fermions and obey non-Abelian anyon statistics. The non-Abelian case is of special interest due to applications in quantum computation and hence we will be distinguishing between the two phases in our studies. Sato et al. [55] have studied and tabulated the different regions of Abelian and non-Abelian 
topological order in our present model of topological superconductivity. We summarize this table below in Table 5.1. While the topological regions do not depend on the Rashba spinorbit coupling, we note that the stability of the superconducting states greatly depends on the strength of the spin-orbit coupling. Even though the Pauli depairing effect becomes increasingly prominent as the Zeeman field increases, strong spin-orbit coupling (and electronphonon coupling) will suppress this effect. Hence in order to obtain stable self-consistent solutions within our model, it is important to have relatively strong spin-orbit coupling. We also note that the inclusion of the Hartree potential effectively alters the regions of topological superconductivity. To obtain the topological regions with the Hartree potential included, in Table 5.1 one must replace $h$ with the effective or "shifted" Zeeman field $\tilde{h}=$ $h+\left(\bar{V}_{\uparrow}^{(H)}-\bar{V}_{\downarrow}^{(H)}\right) / 2$ and $\mu$ with the "shifted" chemical potential $\tilde{\mu}=\mu-\left(\bar{V}_{\uparrow}^{(H)}+\bar{V}_{\downarrow}^{(H)}\right) / 2$ where $\bar{V}_{\sigma}^{(H)}$ is the average spin- $\sigma$ Hartree potential. We demonstrate this in Appendix B.

Figure 5.2 shows the lowest-energy eigenvalue as a function of the Zeeman field with $\mu=3.5 t, \alpha=1 t$ and $\Delta=0.35 t$. The blue curve represents the analytic bulk spectrum given by (5.10) and the red points represent the eigenvalues obtained numerically for an $80 \times 80$ lattice using the SS method. The small oscillations in the spectrum for the $80 \times 80$ lattice are a reflection of the finite system size and become smaller as the system size increases. The close resemblance between the Zeeman-field dependence of the analytical eigenvalues and the numerically determined eigenvalues demonstrates the accuracy and reliability of the SS method. In addition, the large dip in the plot around $h=0.61 t$ indicates a transition from the trivial phase $(h<0.61 t)$ to the non-Abelian topological phase $(h>0.61 t)$. The transition to the second non-Abelian topological phase in region (d) in Table 5.1 will occur around $h=3.52 t$.

For each regime (a) through (d) in Table 5.1, we illustrate the energy spectra for a $50 \times 50$ topological superconducting system in the trivial phase, the Abelian topological phase and the non-Abelian topological phase in Fig. 5.3, 5.5, 5.7 and 5.9. In order to determine the phase of the system, we create edges at $x=0$ and $x=49$ and examine the number of zero-energy bound states that appear. We find that if an even number of zero-energy states appear, the system is in the Abelian topological phase, while if an odd number of bound states appear, the system is in the non-Abelian phase [55]. If there are no zero-energy states, 


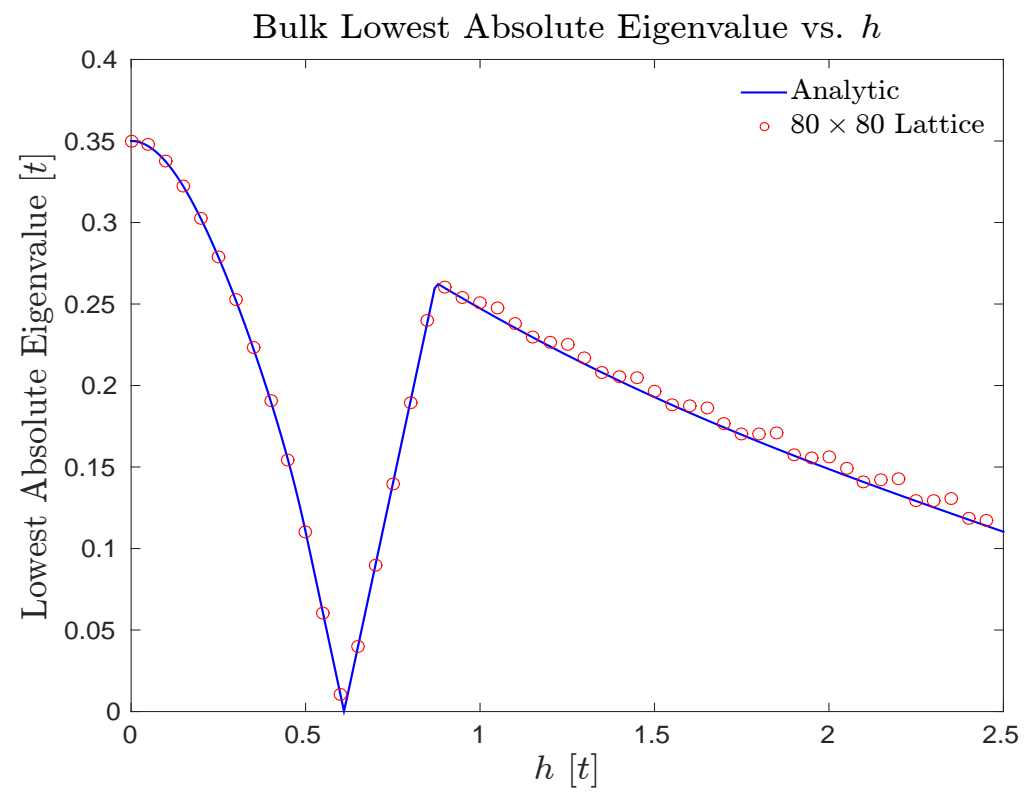

Figure 5.2: Dependence of the lowest absolute bulk eigenvalue on the Zeeman field $h$ for the exact bulk spectrum given by (5.10) (blue curve), and also for an $80 \times 80$ lattice (red points). In both curves, the parameters $\mu=3.5 t, \alpha=1.0 t, \Delta=0.35 t$ have been chosen.

the system is in the trivial phase. Note that due to the periodicity of the Brillouin zone, the states at $k_{y}=\pi$ and $k_{y}=-\pi$ are equivalent. Hence one zero-energy bound state at $k_{y}=\pi$ and one at $k_{y}=-\pi$ correspond to only a single zero-energy bound state. To further justify our claims, we compute the TKNN invariant as a function of $h$ for each regime using Mathematica as shown in Fig. 5.4, 5.6, 5.8 and 5.10. A TKNN number of zero corresponds to the trivial phase, while even and odd TKNN numbers correspond to the Abelian and non-Abelian phases, respectively. 
(a) $\mu \leq-2 t$

\begin{tabular}{cc}
\hline Region & $(-1)^{\mathrm{TKNN}}$ \\
\hline $0<h^{2}<(4 t+\mu)^{2}+\Delta^{2}$ & 1 \\
$(4 t+\mu)^{2}+\Delta^{2}<h^{2}<\mu^{2}+\Delta^{2}$ & -1 \\
$\mu^{2}+\Delta^{2}<h^{2}<(4 t-\mu)^{2}+\Delta^{2}$ & -1 \\
$(4 t-\mu)^{2}+\Delta^{2}<h^{2}$ & 1
\end{tabular}

(b) $-2 t<\mu \leq 0$

\begin{tabular}{cc}
\hline Region & $(-1)^{\mathrm{TKNN}}$ \\
\hline $0<h^{2}<\mu^{2}+\Delta^{2}$ & 1 \\
$\mu^{2}+\Delta^{2}<h^{2}<(4 t+\mu)^{2}+\Delta^{2}$ & 1 \\
$(4 t+\mu)^{2}+\Delta^{2}<h^{2}<(4 t-\mu)^{2}+\Delta^{2}$ & -1 \\
$(4 t-\mu)^{2}+\Delta^{2}<h^{2}$ & 1
\end{tabular}

(c) $0<\mu \leq 2 t$

\begin{tabular}{cc}
\hline Region & $(-1)^{\mathrm{TKNN}}$ \\
\hline $0<h^{2}<\mu^{2}+\Delta^{2}$ & 1 \\
$\mu^{2}+\Delta^{2}<h^{2}<(4 t-\mu)^{2}+\Delta^{2}$ & 1 \\
$(4 t-\mu)^{2}+\Delta^{2}<h^{2}<(4 t+\mu)^{2}+\Delta^{2}$ & -1 \\
$(4 t+\mu)^{2}+\Delta^{2}<h^{2}$ & 1
\end{tabular}

(d) $2 t<\mu$

\begin{tabular}{cc}
\hline Region & $(-1)^{\mathrm{TKNN}}$ \\
\hline $0<h^{2}<(4 t-\mu)^{2}+\Delta^{2}$ & 1 \\
$(4 t-\mu)^{2}+\Delta^{2}<h^{2}<\mu^{2}+\Delta^{2}$ & -1 \\
$\mu^{2}+\Delta^{2}<h^{2}<(4 t+\mu)^{2}+\Delta^{2}$ & -1 \\
$(4 t+\mu)^{2}+\Delta^{2}<h^{2}$ & 1
\end{tabular}

Table 5.1: The regions of trivial, Abelian, and non-Abelian topological order as reported by Sato et al. [55] for the 2-D topological superconductor described by the Hamiltonian in (5.7). $(-1)^{\mathrm{TKNN}}=-1$ corresponds to non-Abelian topological order. 
(a) $h=0$

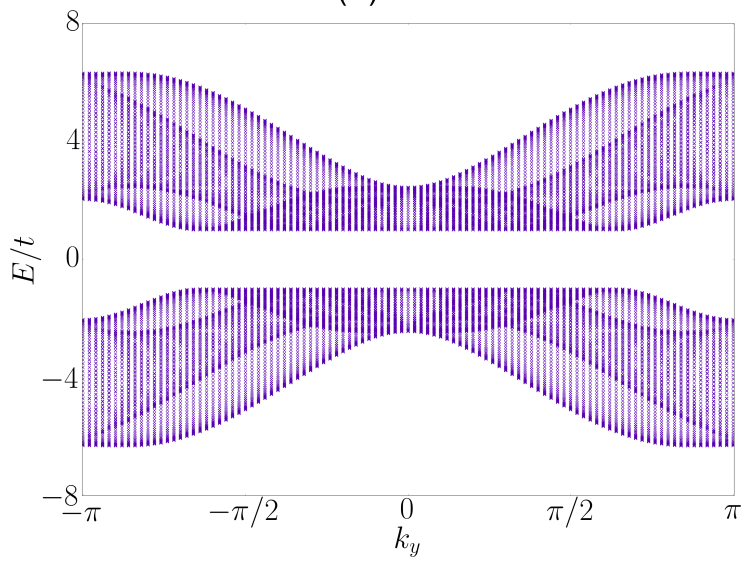

(b) $h=3 t$
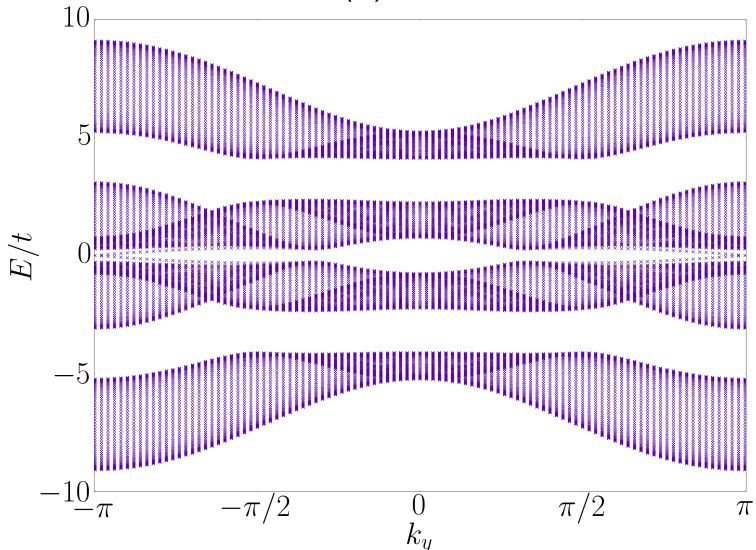

(c) $h=7 t$

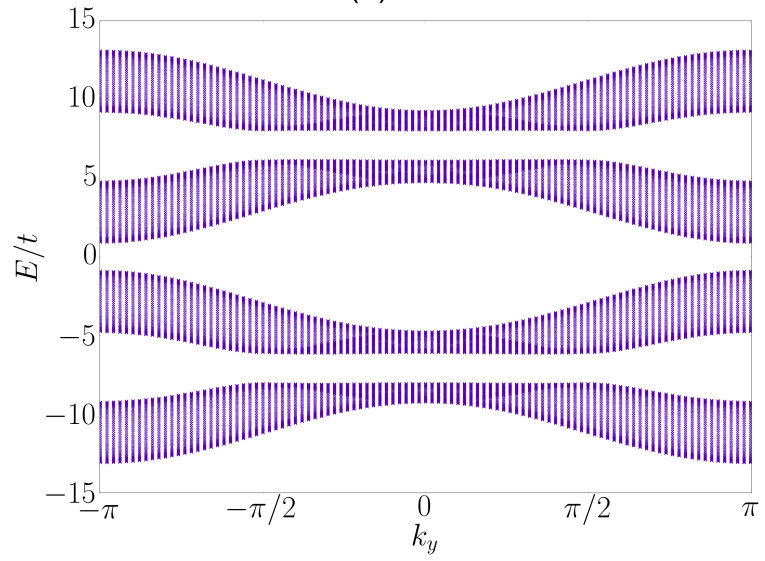

Figure 5.3: Energy spectra for a $50 \times 50$ lattice with edges at $x=0$ and $x=49, \mu=-2.0 t$, and (a) $h=0$ (trivial), (b) $h=3.0 t$ (non-Abelian), (c) $h=7.0 t$ (trivial). The remaining parameters are $\Delta=1.0 t$ and $\alpha=1.0 t$. Note that there is only one non-Abelian region for $\mu=-2.0 t$.

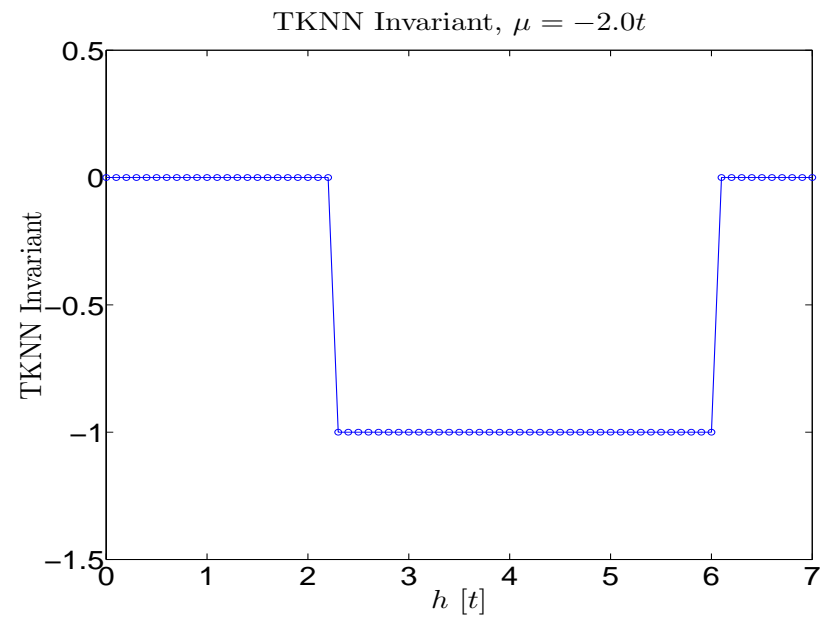

Figure 5.4: TKNN invariant vs. $h$ for $\mu=-2.0 t, \Delta=1.0 t$ and $\alpha=1.0 t$. The transition from the trivial phase to the non-Abelian topological phase occurs at $h \approx 2.24 t$. 
(a) $h=0$

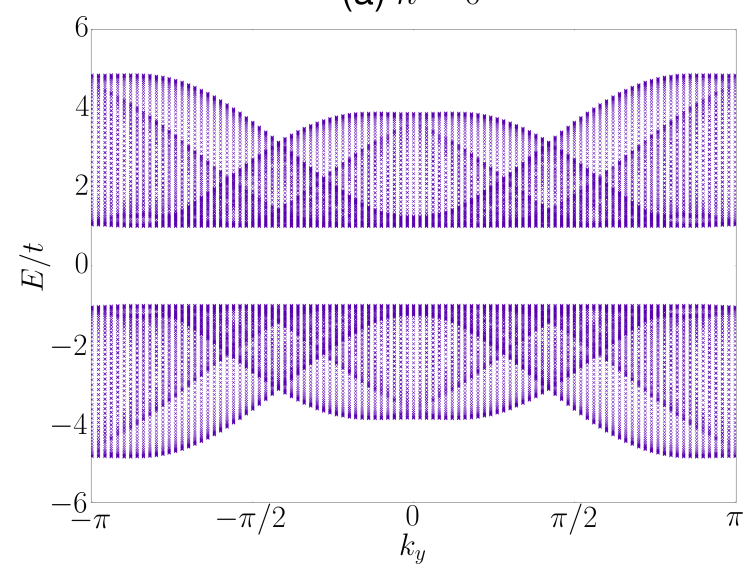

(c) $h=4 t$

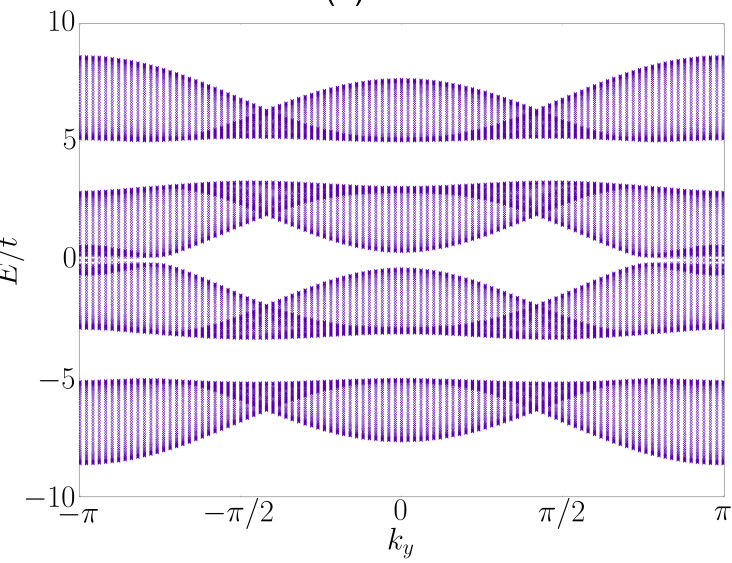

(b) $h=2 t$

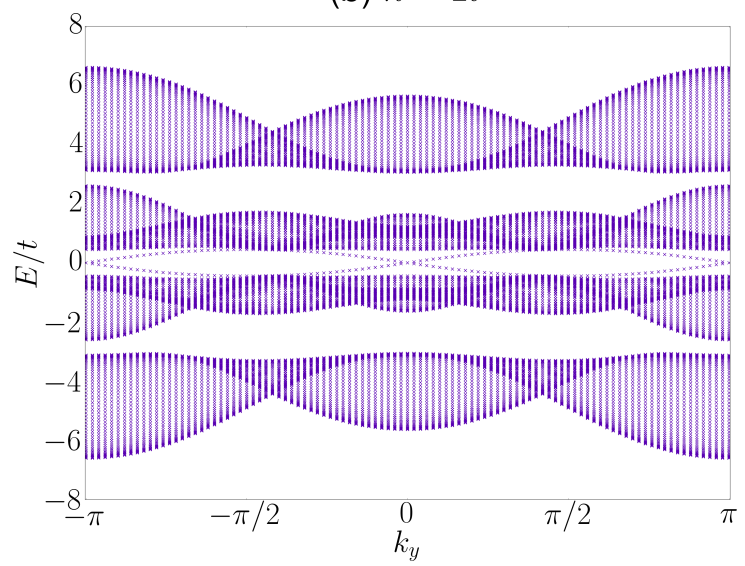

(d) $h=6 t$

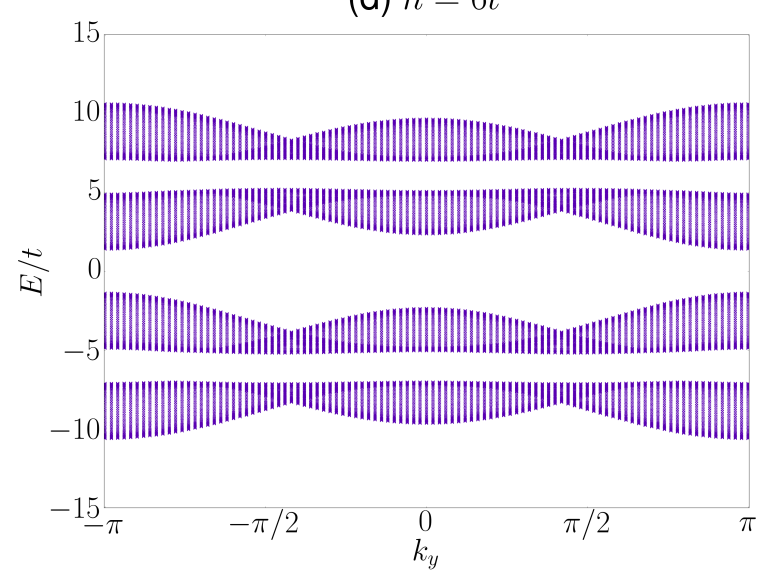

Figure 5.5: Energy spectra for a $50 \times 50$ lattice with edges at $x=0$ and $x=49, \mu=-0.5 t$, and (a) $h=0$ (trivial), (b) $h=2.0 t$ (Abelian), (c) $h=4.0 t$ (non-Abelian), (d) $h=6.0 t$ (trivial). The remaining parameters are $\Delta=1.0 t$ and $\alpha=1.0 t$.

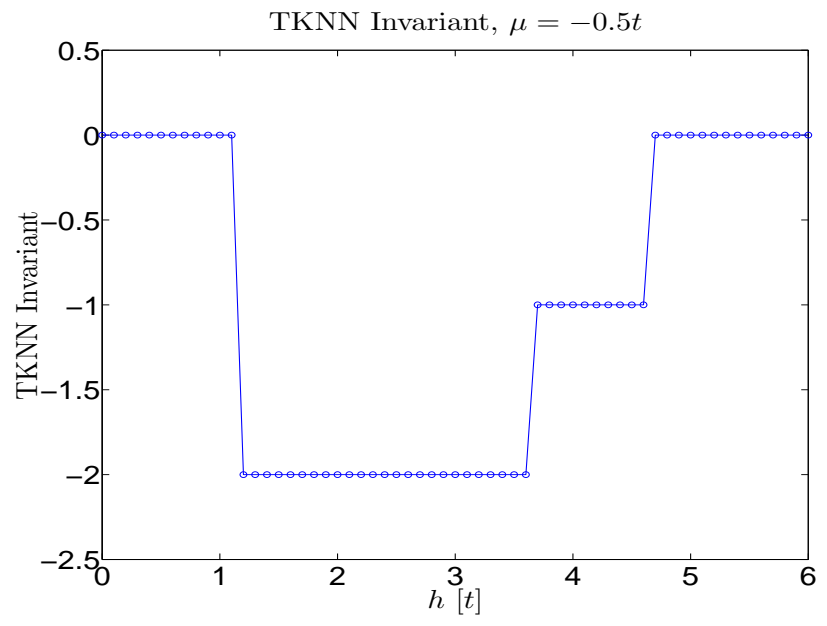

Figure 5.6: TKNN invariant vs. $h$ for $\mu=-0.5 t, \Delta=1.0 t$ and $\alpha=1.0 t$. The transition to the Abelian phase occurs at $h \approx 1.12 t$ while the transition to the non-Abelian phase occurs at $h \approx 3.64 t$. 
(a) $h=0$

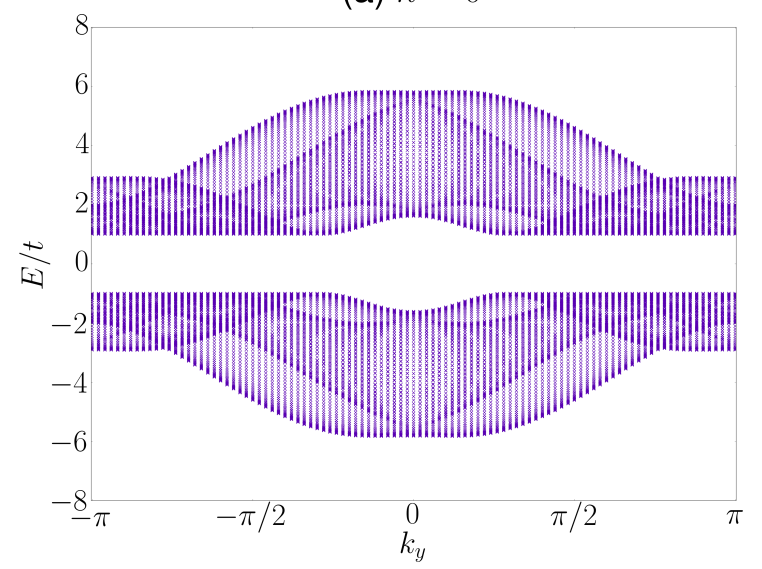

(c) $h=4 t$

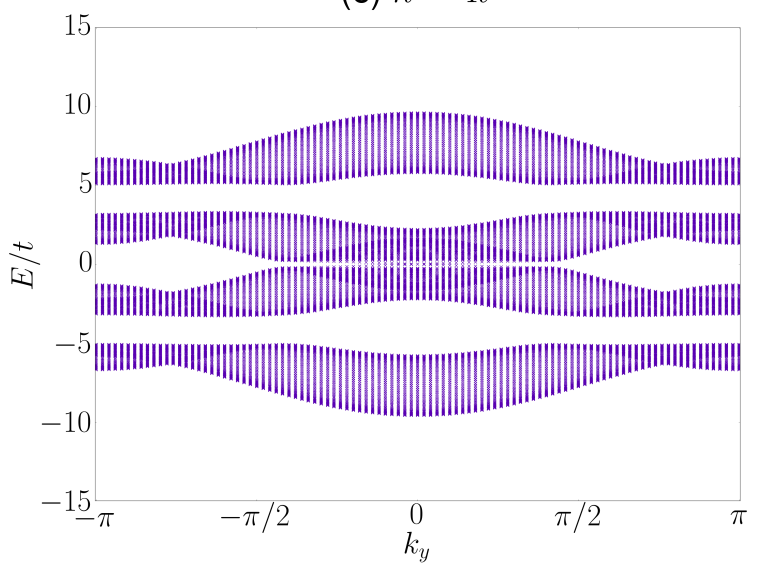

(b) $h=2 t$

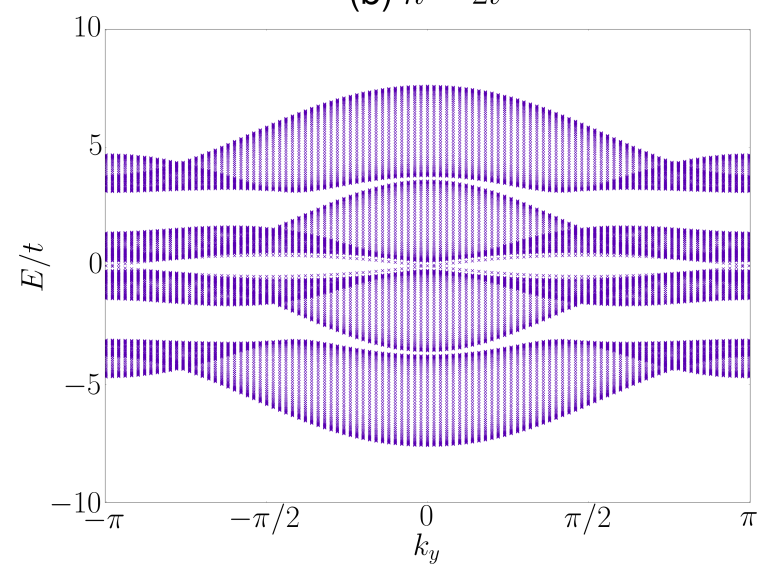

(d) $h=6 t$

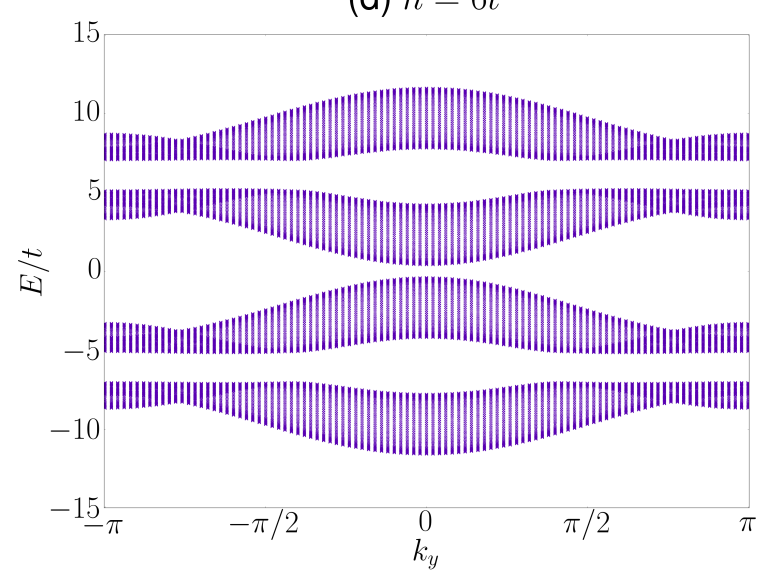

Figure 5.7: Energy spectra for a $50 \times 50$ lattice with edges at $x=0$ and $x=49, \mu=1.5 t$, and (a) $h=0$ (trivial), (b) $h=2.0 t$ (Abelian), (c) $h=4.0 t$ (non-Abelian), (d) $h=6.0 t$ (trivial). The remaining parameters are $\Delta=1.0 t$ and $\alpha=1.0 t$.

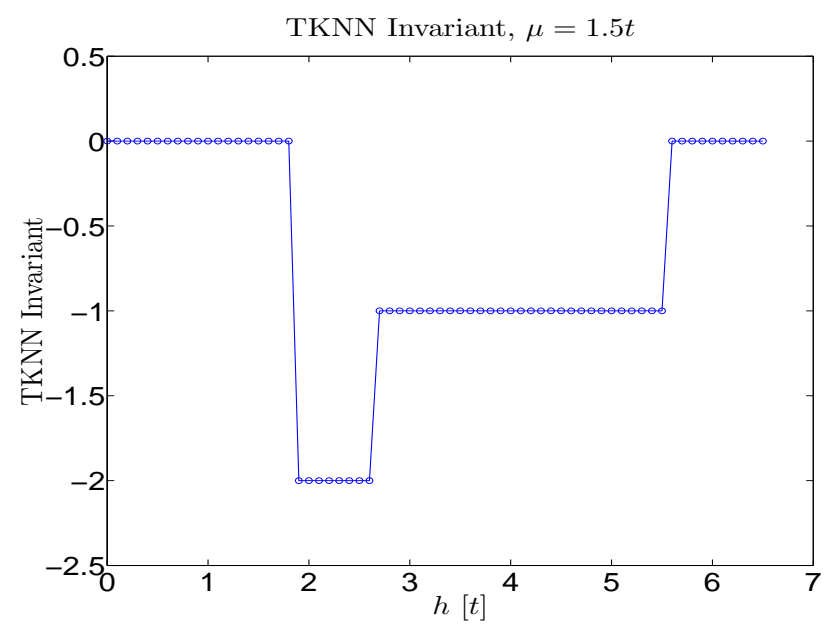

Figure 5.8: TKNN invariant vs. $h$ for $\mu=1.5 t, \Delta=1.0 t$ and $\alpha=1.0 t$. The transition to the Abelian phase occurs at $h \approx 1.80 t$ while the transition to the non-Abelian phase occurs at $h \approx 2.69 t$. 
(a) $h=0$

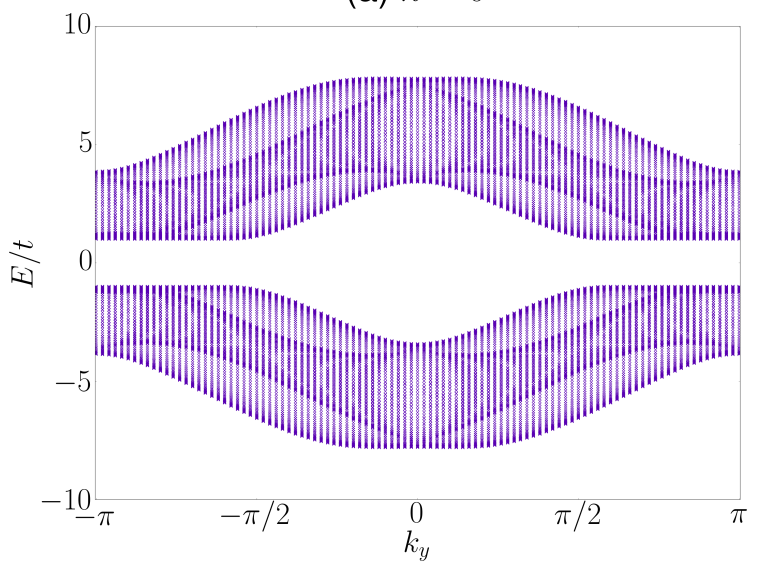

(c) $h=5 t$

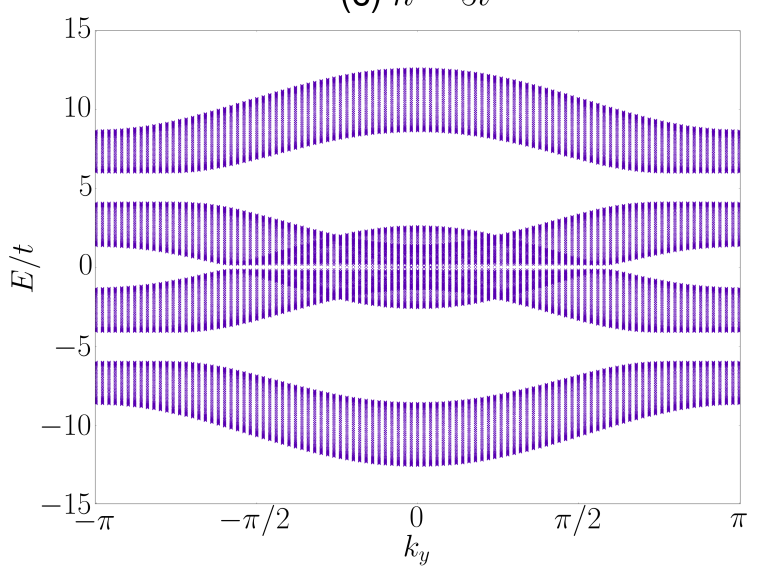

(b) $h=2 t$

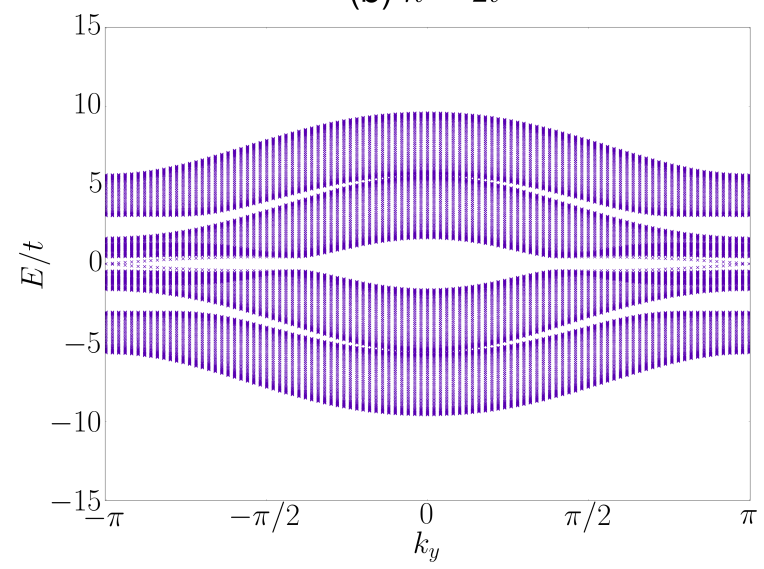

(d) $h=8 t$

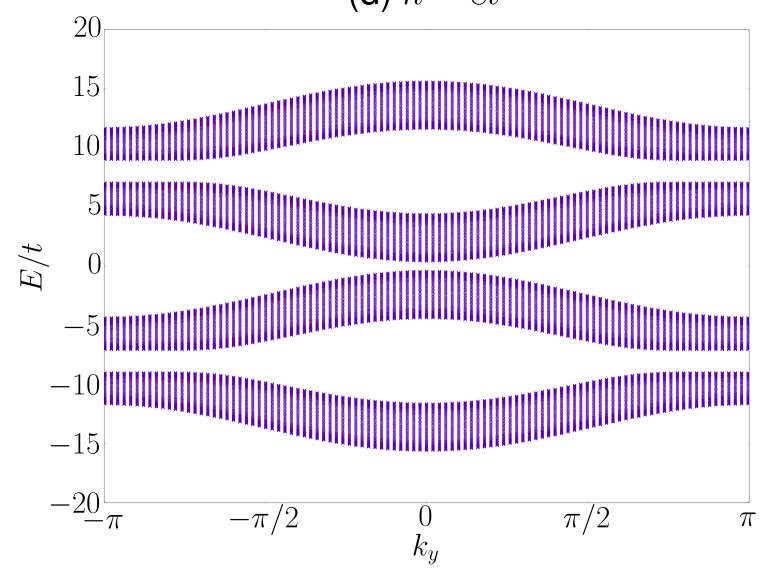

Figure 5.9: Energy spectra for a $50 \times 50$ lattice with edges at $x=0$ and $x=49, \mu=3.5 t$, and (a) $h=0$ (trivial), (b) $h=2.0 t$ (non-Abelian), (c) $h=5.0 t$ (non-Abelian), (d) $h=8.0 t$ (trivial). The remaining parameters are $\Delta=1.0 t$ and $\alpha=1.0 t$.

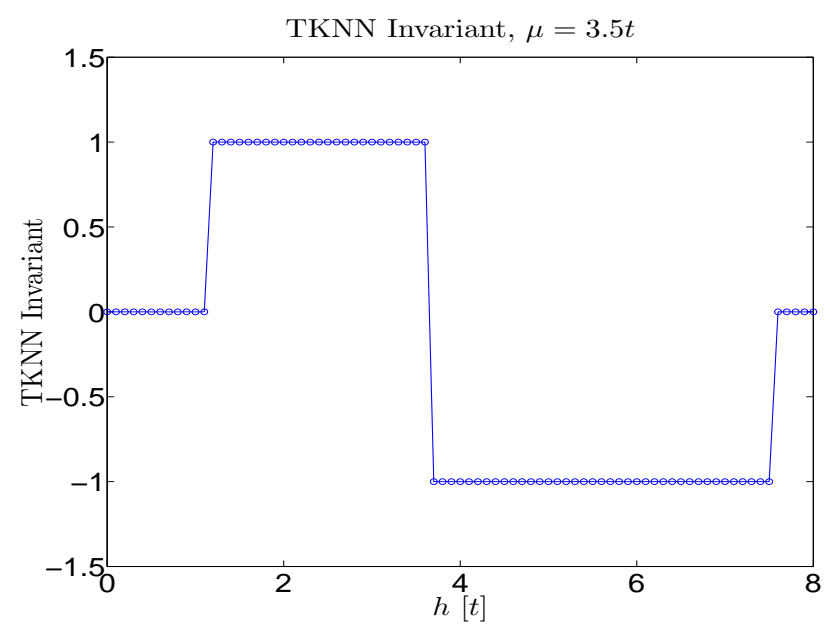

Figure 5.10: TKNN invariant vs. $h$ for $\mu=1.5 t, \Delta=1.0 t$ and $\alpha=1.0 t$. The transition to the first non-Abelian phase occurs at $h \approx 1.12 t$, and the transition to the second occurs at $h \approx 3.64 t$. 


\subsubsection{Self-consistent Studies of Topological Superconductivity}

Using Table 5.1 as a guide, we provide further insight into Abelian and non-Abelian topological superconductivity by obtaining self-consistent solutions to the $\mathrm{BdG}$ equations for the topological superconductor described by the Hamiltonian (5.1). Due to the high numerical resources required, self-consistent calculations were performed on several Westgrid computing clusters. Initially, we solve only the order parameter self-consistently and obtain a selfconsistent version of Fig. 5.2 for a $50 \times 50$ lattice shown in Fig. 5.11. Using the Chebyshev polynomial expansion method, the order parameter was self-consistently determined with periodic boundary conditions (PBC) for $\mu=3.5 t, \alpha=1.0 t$, and uniform electron-phonon coupling constant $U_{i i} \equiv U=-5.0 t$. Figure 5.11 (b) illustrates the lowest absolute eigenvalue vs. the Zeeman field $h$ for the converged system and shows the transition to the non-Abelian topological phase occurring around $h=0.75 t$, where $\Delta=0.515 t$. Again we note that it is important to have sufficiently strong spin-orbit coupling in order to obtain stable self-consistent superconducting states. Figure 5.11 (a) demonstrates this for Zeeman fields of $h \gtrsim 1.5 t$, the Rashba spin-orbit coupling value of $\alpha=1.0 t$ is no longer sufficient for this parameter set and both $\Delta$ and the lowest absolute eigenvalue approach zero.

(a)

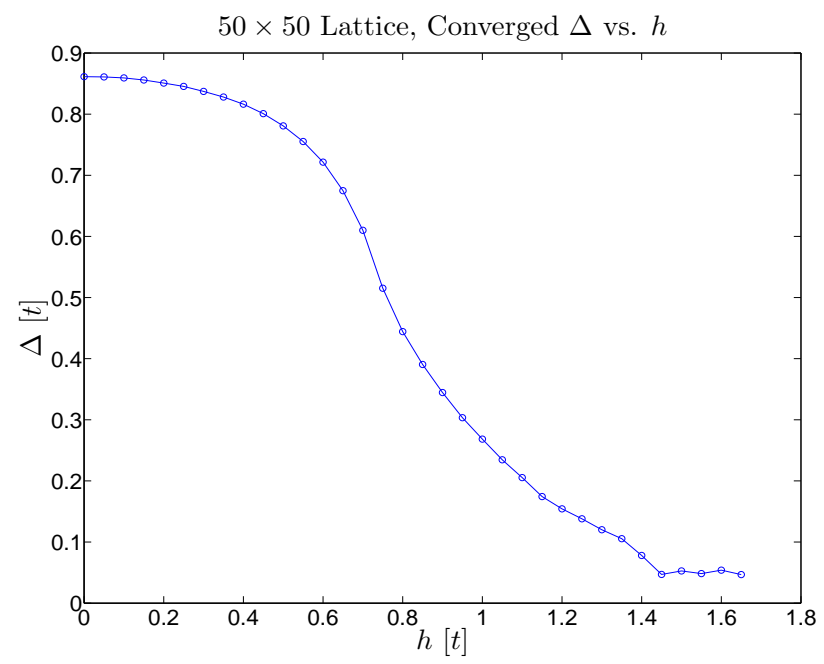

(b)

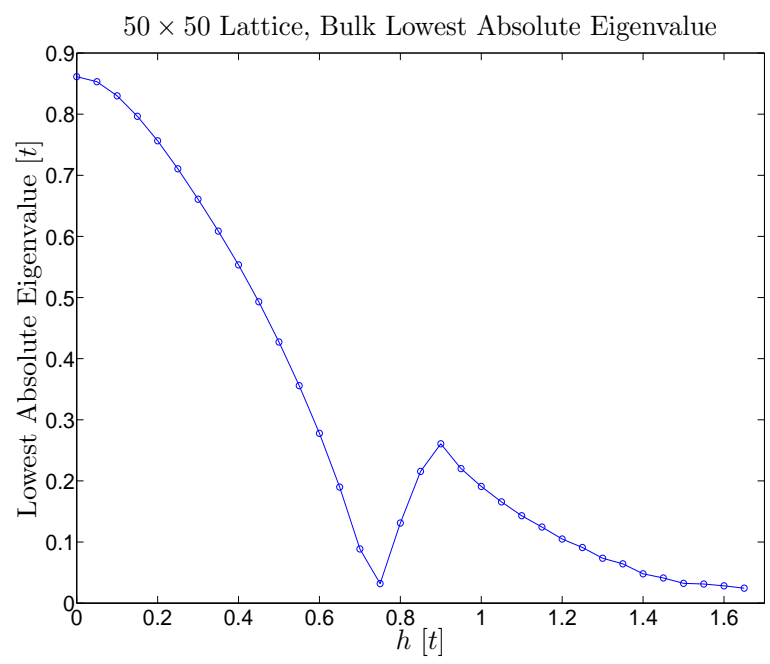

Figure 5.11: (a) Dependence of the superconducting order parameter on the Zeeman field $h$ for a $50 \times 50$ lattice for $\mu=3.5 t, \alpha=1.0 t$ and $U=-5.0 t$. (b) Dependence of the lowest absolute eigenvalue on the Zeeman field for the system described in (a). Transition from the trivial phase to the non-Abelian topological phase occurs around $h=0.75 t$. 
To obtain further self-consistent solutions, we investigate possible parameter sets by determining how the order parameter $\Delta$ varies with the coupling constant $|U|$ for certain regions in Table 5.1. Figure 5.12 (a) through (c) demonstrates how the order parameter varies with the absolute value of the coupling constant, first neglecting the Hartree potential for a $50 \times 50$ system with PBC. As the choice of coupling constant for these topological superconducting systems is often unclear, these results provide us with a useful reference in selecting a coupling constant that does not push the system out of the topological region and into the trivial region. For example, in Fig. 5.12 (a) we have selected $\mu=-3.0 t, h=1.5 t$ and $\alpha=1.5 t$ and so the transition from the topological phase to the trivial phase will occur for $\Delta>1.12 t$. Hence the selection of $|U|>6.2 t$ will cause the system to transition into the trivial phase. For Fig. 5.12 (b) with $\mu=1.0 t, h=1.5 t$ and $\alpha=2.0 t$, the transition out of the Abelian topological phase will also occur for $\Delta>1.12 t$ and hence we should choose $|U|<5.8 t$. Lastly for Fig. $5.12(\mathrm{c})$, we have chosen $\mu=3.5 t, h=1.0 t$ and $\alpha=1.0 t$ and so the transition from the non-Abelian topological phase to the trivial phase will occur for $\Delta>0.87 t$ so we must select $|U|<6.0 t$.

In order to illustrate how the topological regions change with the inclusion of the Hartree potential, we repeat the calculations performed in Fig. 5.12, this time also self-consistently solving for the Hartree potential. Figure 5.13 shows the result of these calculations, beginning with Fig. 5.13 (a) in which we have chosen $\tilde{\mu}=-3.0 t, h=1.5 t, \alpha=1.5 t$. This system transitions now from the non-Abelian topological region to the trivial region around $|U|=$ $4.8 t$ where we have found $\tilde{h}=1.09755 t$. Figure $5.13(\mathrm{~b})$ shows $\tilde{\mu}=1.0 t, h=1.5 t$ and $\alpha=2.0 t$ which has a very narrow region of Abelian topological superconductivity. The transition to the trivial phase will occur for $|U| \geq 3.2 t$, where at $|U|=3.2 t$ we have found $\tilde{h}=1.02766 t$. Lastly when $\tilde{\mu}=3.5 t, h=1.0 t$ and $\alpha=1.0 t$ as in Fig. 5.13 (c), we do not leave the non-Abelian topological region until $|U| \geq 5.2 t$ where at $|U|=5.2 t$ we have obtained $\tilde{h}=0.78612 t$. Hence in general, due to the effective shifting of the Zeeman field, we find that one must choose a lower electron-phonon coupling constant when the Hartree potential is also solved self-consistently alongside the order parameter.

We next examine the behaviour of Majorana fermions in the system by introducing edges in the $x$-direction within our square lattice. First, we neglect the Hartree potential and 
(a)

$\Delta$ vs. $|U|$ for $50 \times 50$ Lattice, $\mu=-3.0 t$



(b)

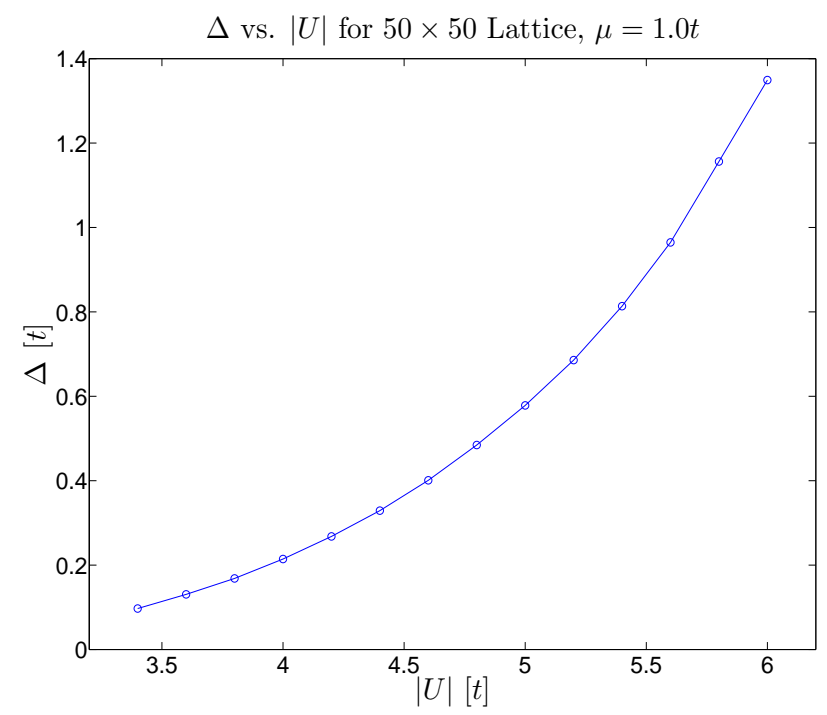

(c)

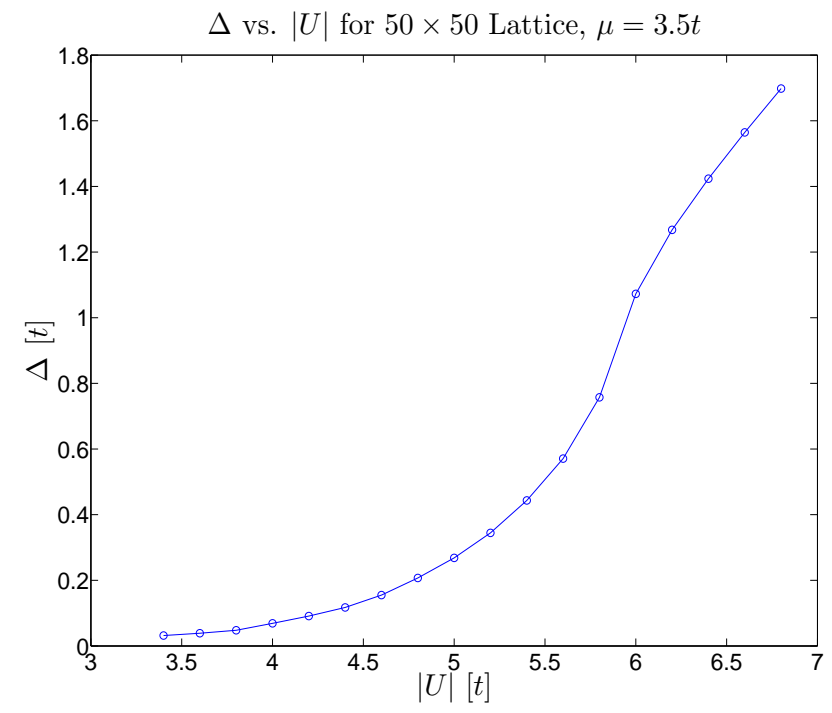

Figure 5.12: Value of the order parameter $\Delta$ vs. the absolute value of the coupling constant $|U|$ for a $50 \times 50$ lattice with PBC and (a) $\mu=-3 t, h=1.5 t, \alpha=1.5 t$, (b) $\mu=1.0, h=1.5 t, \alpha=2.0 t$ and (c) $\mu=3.5 t, h=1.0 t, \alpha=1.0 t$. 
(a)

$\Delta$ vs. $|U|$ for $50 \times 50$ Lattice with HP, $\tilde{\mu}=-3.0 t$

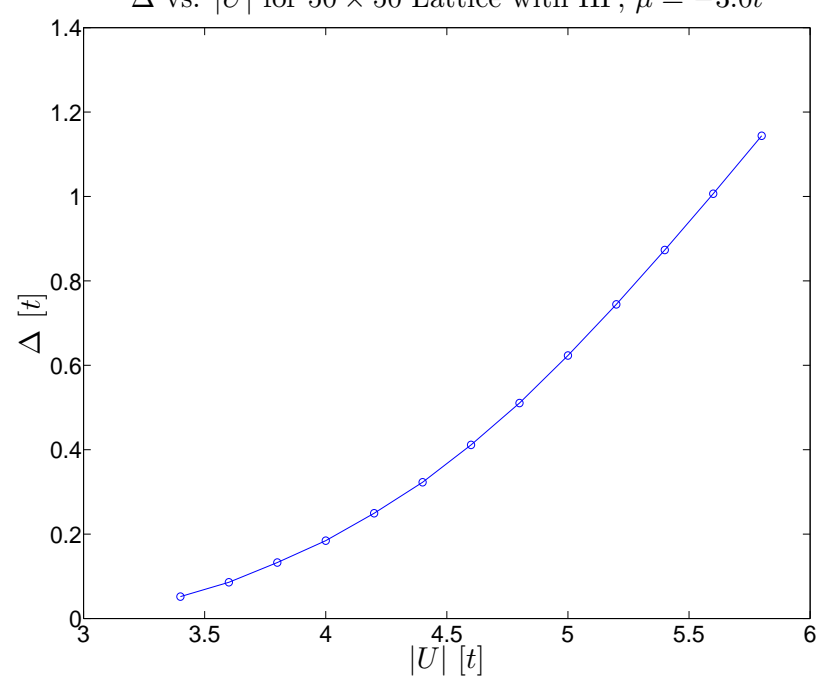

(b)

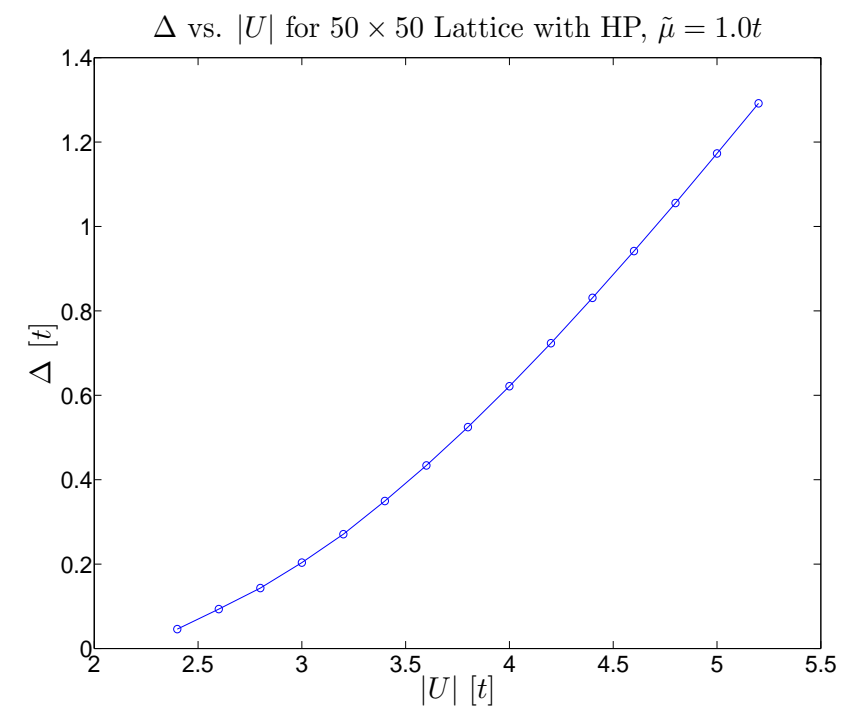

(c)

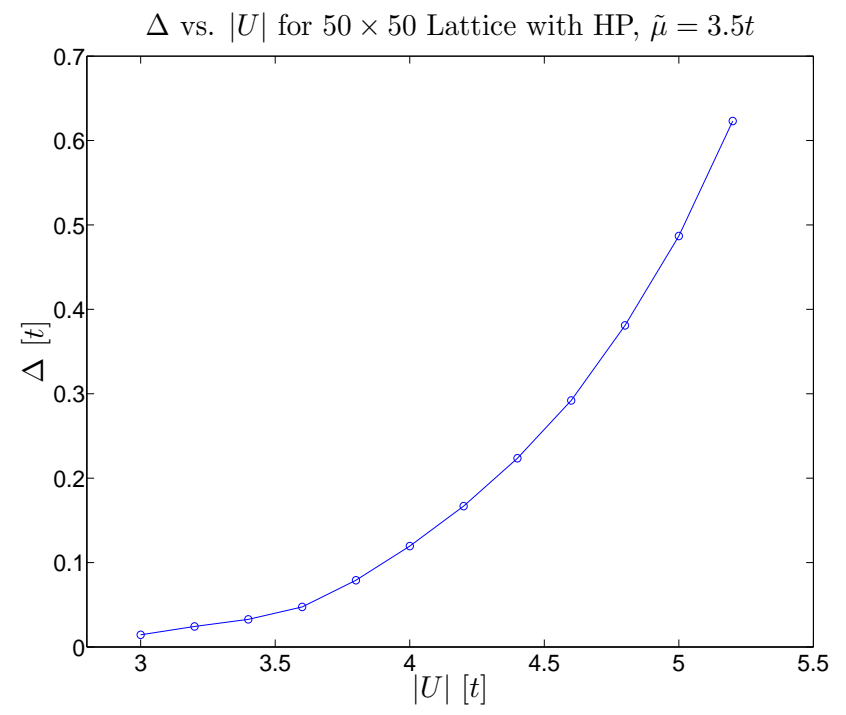

Figure 5.13: Value of the order parameter $\Delta$ vs. the absolute value of the coupling constant $|U|$ with the inclusion of the Hartree potential for a $50 \times 50$ lattice with PBC and (a) $\tilde{\mu}=-3 t, h=1.5 t$, $\alpha=1.5 t$, (b) $\tilde{\mu}=1.0 t, h=1.5 t, \alpha=2.0 t$ and (c) $\tilde{\mu}=3.5 t, h=1.0 t, \alpha=1.0 t$. 
self-consistently solve for the order parameter in an $80 \times 80$ system in the non-Abelian topological phase with edges at $x=0$ and $x=79, \mu=3.0 t, h=1.5 t$ and $\alpha=1.5 t$. Figure 5.12 (a) allows us to easily select an appropriate coupling constant for the system as both $\mu=-3.0 t$ and $\mu=3.0 t$ will produce the same order parameter. Thus, we select a coupling constant of $U=-5.5 t$. After convergence, the lowest-energy eigenvalues are obtained using the SS method and are plotted as a function of index in Fig. 5.14 (a). In the presence of edges in the $x$-direction, we clearly see the appearance of in-gap, Majorana bound states represented by the lowest absolute eigenvalue of the system, $E=2.67936 \times 10^{-6}$. Moreover, as shown in Fig. 5.15 (a), plotting the magnitude squared of the wavefunction shows that these zero-energy bound states are indeed localized at the edges of the system with one Majorana fermion present at each edge. Including the Hartree potential, we must reduce the coupling constant to $U=-4.2 t$ to account for the shifted $h$ of the system. Using input parameters of $\tilde{\mu}=3.0 t, h=1.5 t$ and $\alpha=1.5 t$ to stay in the nontrivial region, we obtain a shifted $h$ of $\tilde{h}=1.10933 t$ after convergence. The lowest-energy eigenvalues are plotted in Fig. 5.14 (b), again clearly showing zero-energy Majorana bound states represented by our lowest absolute eigenvalue of $E=3.08687 \times 10^{-4}$. We note that it was not possible to use the same coupling constant for both the case where the Hartree potential was included and the case where it was not included. As Fig. 5.14 (c) demonstrates, using a coupling constant of $U=-4.2 t$ without converging the Hartree potential results in no Majorana bound states despite the presence of edges. This is because selecting $U=-4.2 t$ results in a converged superconducting order parameter that is extremely small, and hence the spectral gap becomes negligible resulting in a wavefunction that spreads throughout the entire system as shown in Fig. 5.15 (b). 
(a)

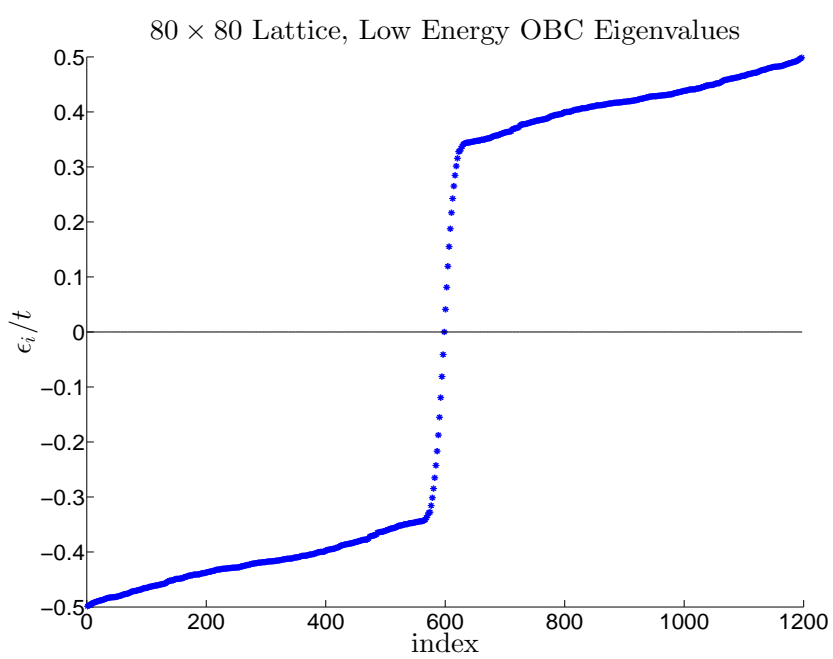

(b)

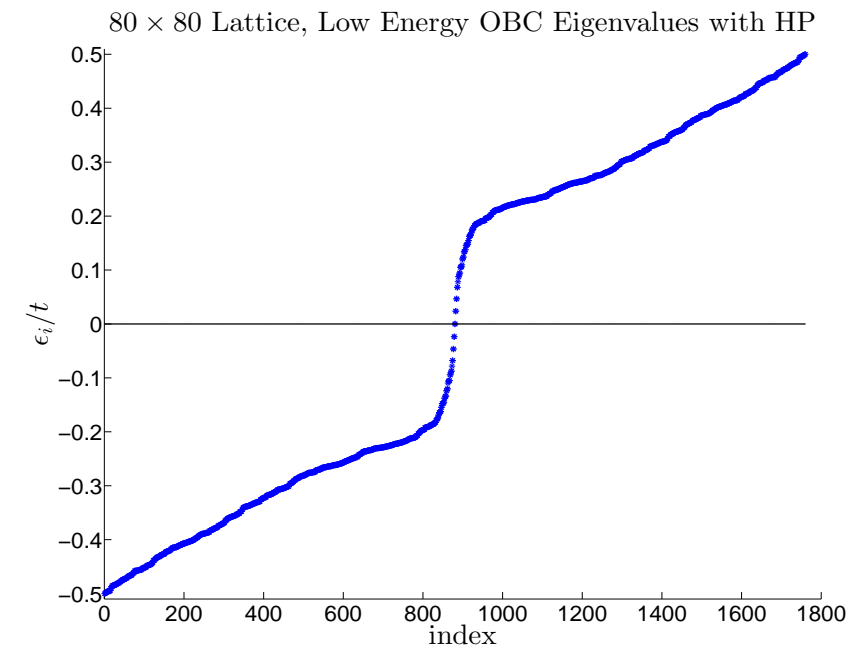

(c)

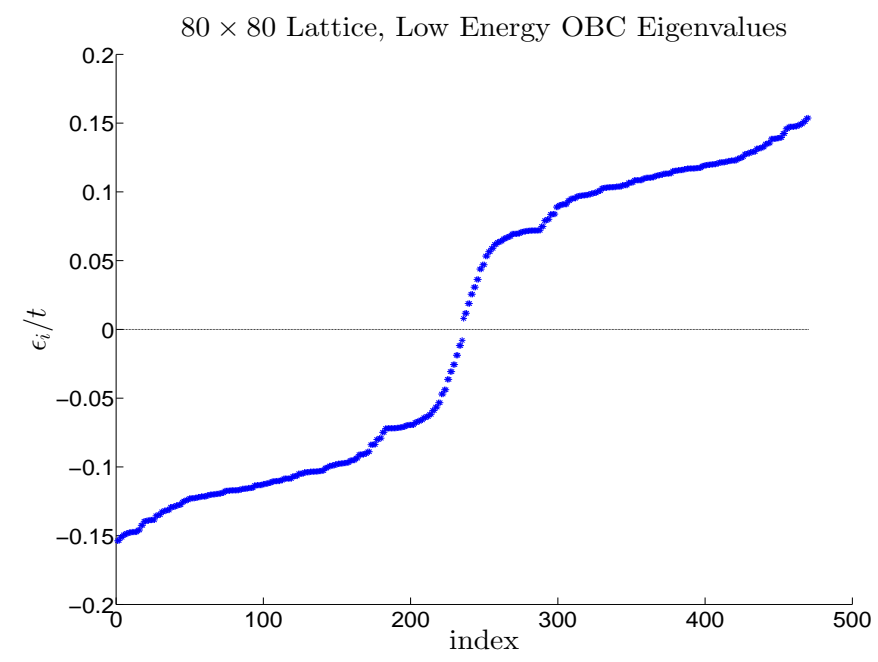

Figure 5.14: (a) Eigenvalues of the BdG Hamiltonian (5.9) for an $80 \times 80$ system neglecting the Hartree potential with $\mu=3 t, h=1.5 t, \alpha=1.5 t, U=-5.5 t$ and surfaces at $x=0$ and $x=79$. (b) Eigenvalues for the system including the Hartree potential with $U=-4.2 t$. The remaining parameters are the same as in (a). (c) Eigenvalues for the $80 \times 80$ system neglecting the Hartree potential with $U=-4.2 t$. Selecting $U=-4.2 t$ results in a spectral gap too small for the observation of Majorana zero modes. The remaining parameters are the same as in (a). 
(a)

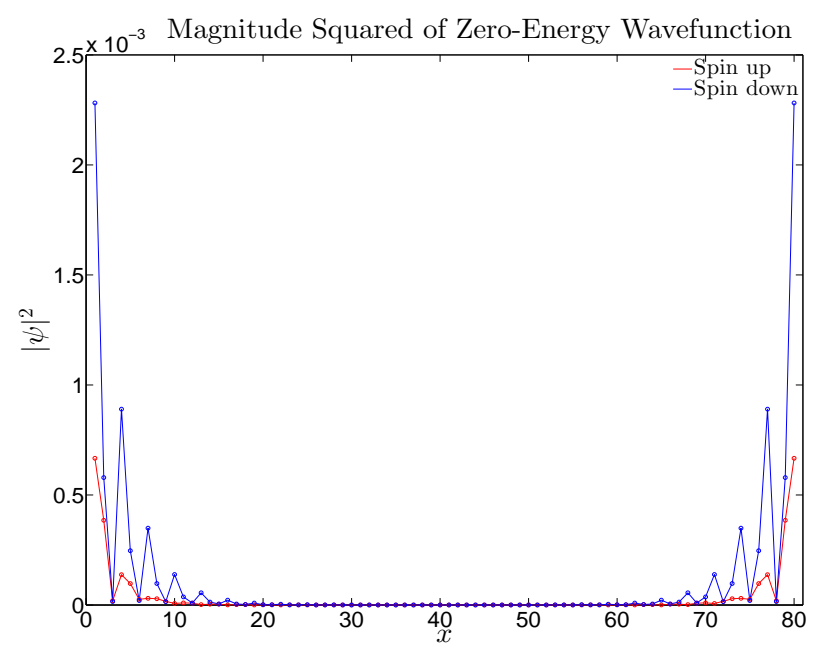

(b)

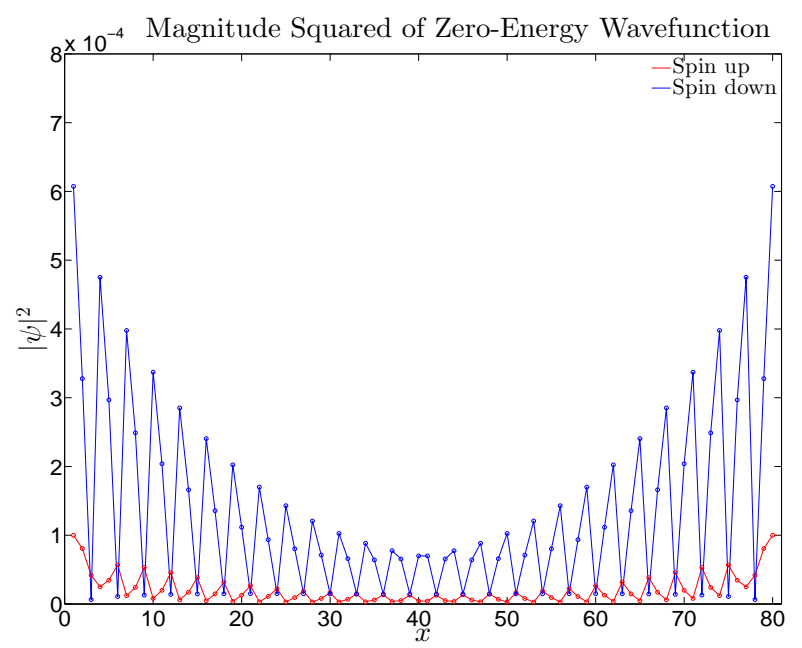

Figure 5.15: (a) Magnitude squared of the zero-energy wavefunction corresponding to the lowestenergy eigenvalue shown in Fig. 5.14 (a). One Majorana fermion is present on each edge of the system. (b) Magnitude squared of the wavefunction corresponding to the lowest-energy eigenvalue shown in Fig. 5.14 (c). The wavefunction is no longer localized at each edge of the system due to the exceedingly small spectral gap.

\subsubsection{The Effects of Temperature}

In this section we are motivated by a momentum-space study of impurity effects in an $s$-wave topological superconductor performed by Nagai, Ota and Machida [1]. First, we investigate the $T_{c}$ behaviour presented in Ref. [1] where two systems in the non-Abelian topological phase with odd TKNN number were considered. The Hartree potential was not included in the work of Ref. [1]. In the low magnetic field region, a system with $\mu=3.5 t, h=1.0 t, \alpha=1.0 t$ and $U=-5.6 t$ was considered, in addition to a system in a higher magnetic field region with $\mu=3.5 t, h=2.0 t, \alpha=1.0 t$ and $U=-8.0 t$. The authors demonstrated that in the lower Zeeman field region of $h=1.0 t$, superconductivity disappears at a critical temperature of $T_{c}=0.16 t$, whereas in the higher Zeeman field region with $h=2.0 t$, superconductivity disappears at a much lower value of $T_{c}=0.10 t$. For a $40 \times 40$ lattice, we have performed both the low and high field region calculations as shown in Fig. 5.16. In contrast to the results presented in Fig. 5 in Ref. [1], we obtain $T_{c}$ values of $T_{c}=0.23 t$ and $T_{c}=0.20 t$ for the low and high Zeeman field regions shown in Fig. 5.16 (a) and (b), respectively. This may be mainly because the order parameter at zero temperature is larger for $h=2.0 t(\sim 0.8 t)$ than for 
$h=1.0 t(\sim 0.57 t)$ in our self-consistent results, while the zero-temperature order parameter has the value of $\sim 0.4$ for both $h=1.0 t$ and $h=2.0 t$ in the work presented in [1]. The difference in the order parameter values between our BdG results and the results of [1] stems from the fact that the Green function approach used in [1] depends on a cutoff Matsubara frequency, while the BdG theory does not rely on such a cutoff parameter. Equating the zero-temperature order parameters for both systems, we obtain Fig. 5.16 (c) and Fig. 5.16 (d) in which we have calculated critical temperatures of $T_{c}=0.15 t$ and $T_{c}=0.11 t$ in the low and high Zeeman field regions, respectively. Hence we indeed find that a larger Zeeman field causes an increased sensitivity to temperature due to the fact that the spins near the Fermi level are well polarized. For completeness, we have calculated the critical temperature for the low Zeeman field case, $\tilde{\mu}=3.5 t, h=1.0 t, \alpha=1.0 t$ and $U=-4.8 t$, with the addition of the Hartree potential and we find that the critical temperature increases slightly to a value of $T_{c}=0.19 t$ as shown in Fig. 5.16 (e). This is expected as the presence of the Hartree potential effectively reduces the magnitude of the Zeeman field. Lastly, in 5.16 (f) we compute the critical temperature for a system in the Abelian topological phase with even TKNN number for parameters $\mu=1.0 t, h=1.5 t, \alpha=2.0 t$ and $U=-4.5 t$, neglecting the Hartree potential. Despite the fact that $h$ is larger than in the case of Fig. 5.16 (c), the strong spin-orbit coupling suppresses the impact of temperature on the system and we obtain a slightly higher critical temperature of $T_{c}=0.16 t$. 
(a)

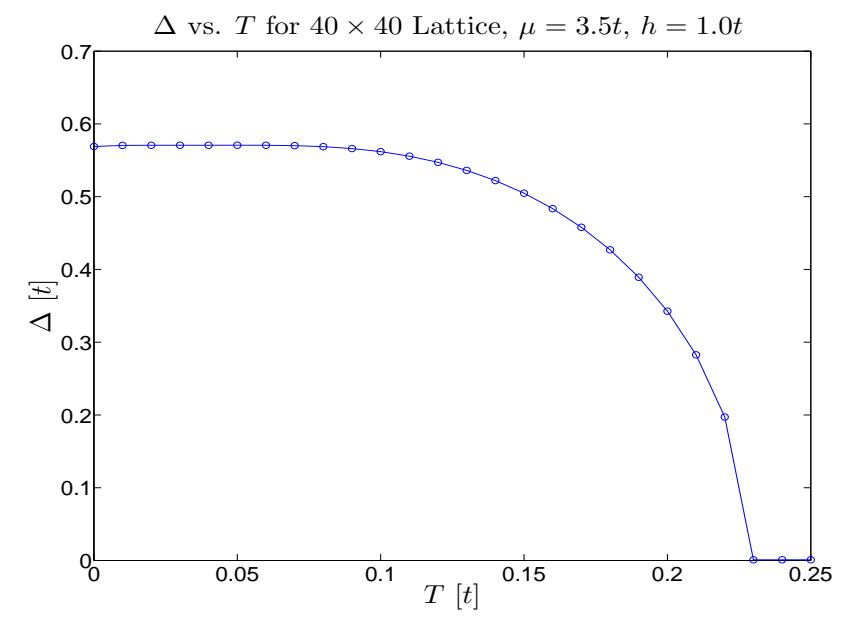

(c)

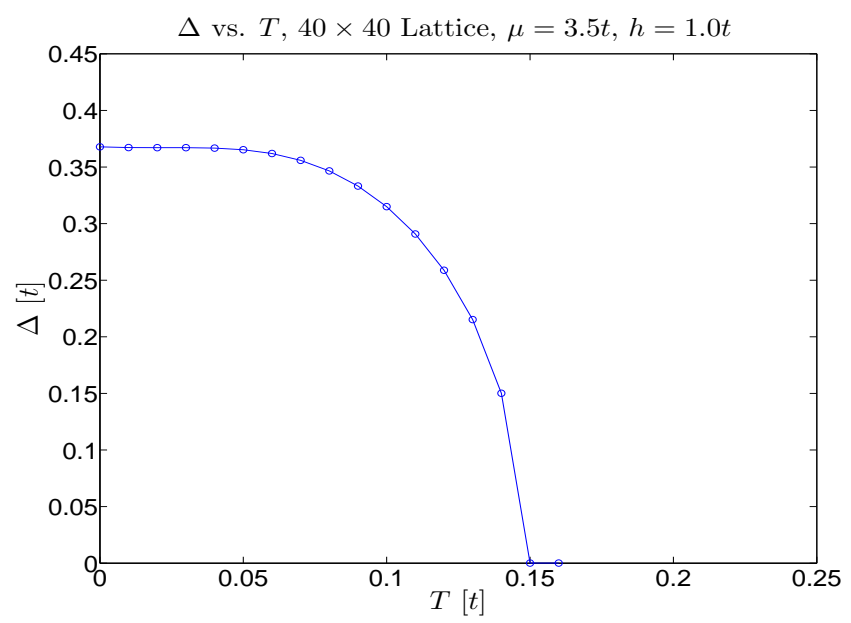

(e)

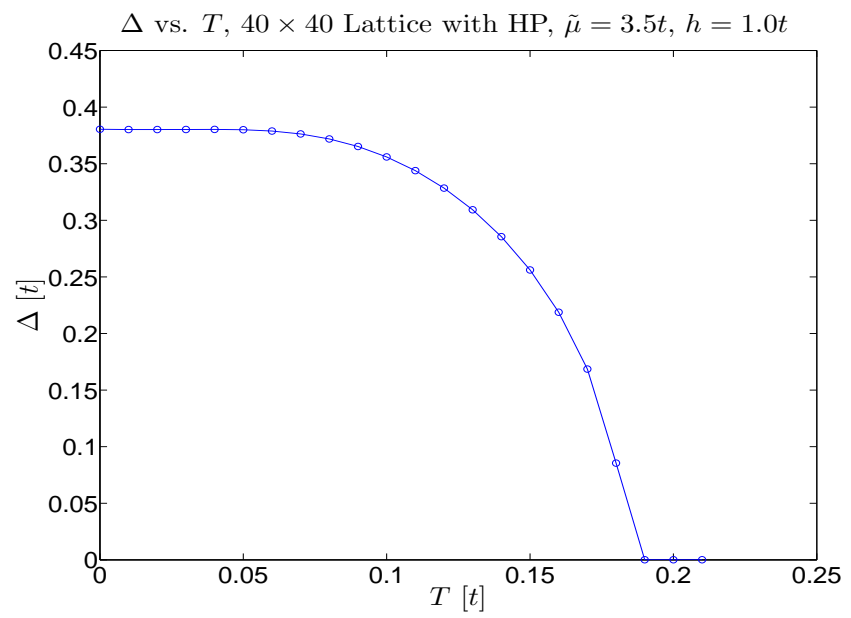

(b)

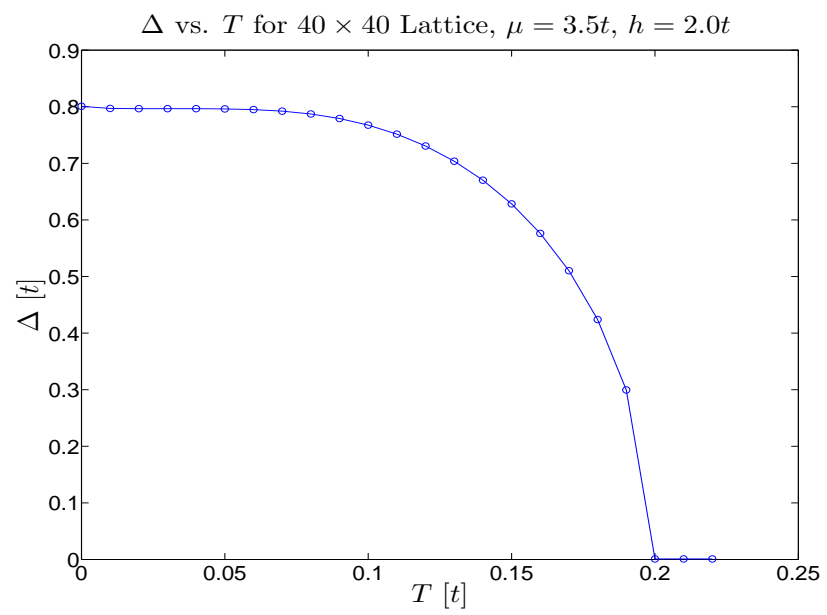

(d)

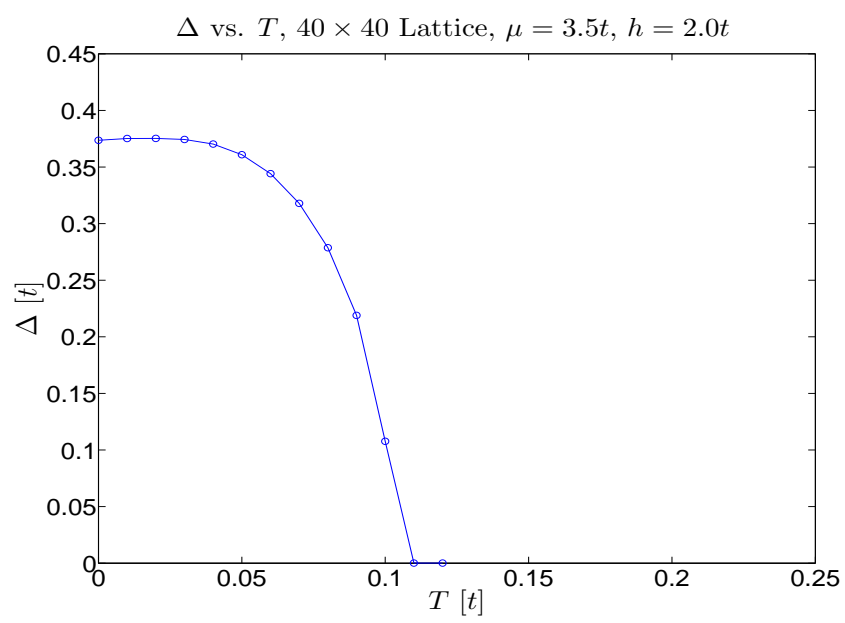

(f)

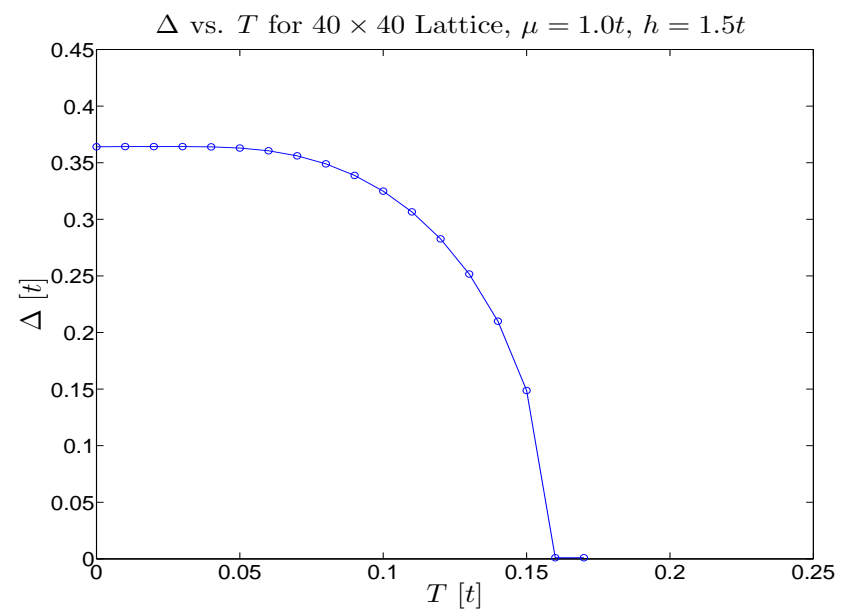

Figure 5.16: Temperature dependence of the order parameter for a $40 \times 40$ lattice with (a) $\mu=3.5 t$, $h=1.0 t, \alpha=1.0 t, U=-5.6 t$, (b) $\mu=3.5 t, h=2.0 t, \alpha=1.0 t, U=-8.0 t$, (c) $\mu=3.5 t, h=1.0 t$, $\alpha=1.0 t, U=-5.25 t$, (d) $\mu=3.5 t, h=2.0 t, \alpha=1.0 t, U=-7.38 t$, (e) $\tilde{\mu}=3.5 t, h=1.0 t, \alpha=1.0 t$ and $U=-4.8 t$ with the Hartree potential included, and (f) $\mu=1.0 t, h=1.5, \alpha=2.0 t$ and $U=-4.5 t$. 


\subsubsection{The Effects of a Single Non-magnetic Impurity}

We would now like to consider the effects of depositing a single non-magnetic impurity at the center of a $64 \times 64$ lattice for a system with even TKNN number, and a system with odd TKNN number in order to observe the difference in impurity effects between the case of Abelian and non-Abelian TSC. To this end, neglecting the Hartree potential, we first study a system with $\mu=3.5 t, h=1.0 t, \alpha=1.0 t$ and coupling constant $U=-5.2 t$ with a single attractive impurity potential $V_{\mathrm{imp}}=-2.0 t$ deposited at site $(32,32)$. The clean system is in the non-Abelian topological phase and upon computing the TKNN number, we find that $\nu=1$. The converged order parameter for the system is illustrated in Fig. 5.17 showing significant suppression at the impurity site despite the relatively weak impurity, reminiscent of $p$-wave superconductivity as predicted by Sato et al. [55]. Figure 5.18 then illustrates the Abelian topological phase with $\mu=1.0 t, h=1.5 t, \alpha=2.0 t, U=-4.445 t$ and $V_{\mathrm{imp}}=-2.0 t$ where a TKNN number of $\nu=-2$ has been computed in the clean system. In this even TKNN system, despite the larger Zeeman field the order parameter shows little suppression at the impurity site, however a large area of the lattice becomes affected by the impurity, showing oscillations around the impurity site in contrast to Fig. 5.17.

Lastly, we can observe the impurity effects in these two systems through the local density of states (LDOS). Figure 5.19 illustrates the spin-up and spin-down LDOS in the odd TKNN case with the same parameters as in Fig. 5.17. The clean system is represented by the blue curve whereas the impurity system with $V_{\text {imp }}=-2.0 t$ is represented by the red curve. In the case of non-Abelian TSC, there is one impurity bound state present corresponding to the TKNN number $\nu=1$ which can be seen in the LDOS very near the gap edge. Figure 5.20 then shows the even TKNN case with the same parameters as in Fig. 5.18. Due to the TKNN number taking on a value of $\nu=-2$, two impurity bound states are present, with one bound state being well within the gap, clearly visible in the LDOS, and the second bound state appearing near the gap edge. In addition to both systems having an almost identical bulk order parameter values as can be seen in Fig. 5.17 and Fig. 5.18, the impurity is nonmagnetic implying that the Zeeman field and Rashba spin-orbit coupling have no bearing on the impurity effects observed. Therefore, we conclude that the impurity effects observed 
between the two systems must be a consequence of the underlying topology.

$64 \times 64$ Lattice, $\mu=3.5 t, V_{i m p}=-2.0 t$

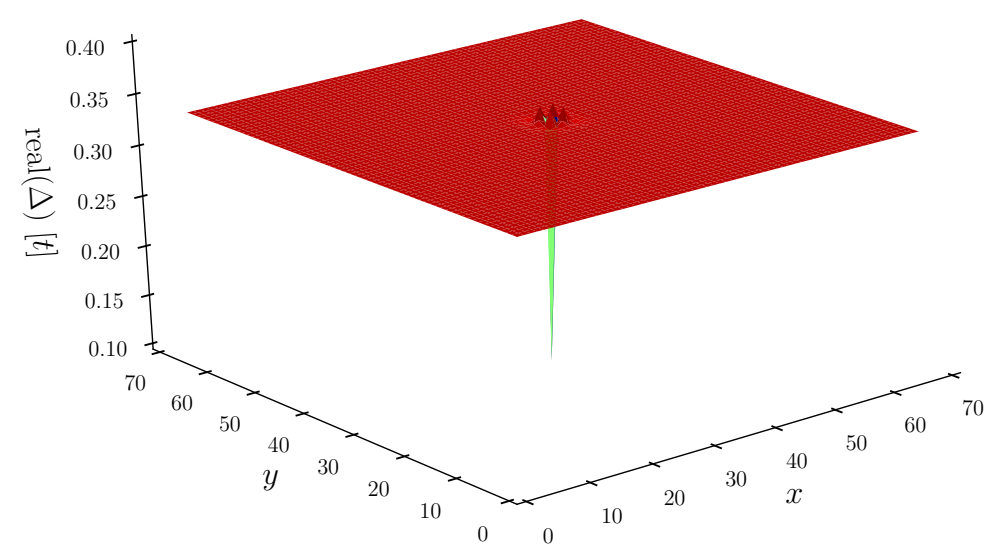

Figure 5.17: Real part of the order parameter for a single non-magnetic attractive impurity potential $V_{\text {imp }}=-2.0 t$ deposited at the center of a $64 \times 64$ lattice with $\mu=3.5 t, h=1.0 t, \alpha=1.0 t$ and $U=-5.2 t$ corresponding to $\nu=1$.

$64 \times 64$ Lattice, $\mu=1.0 t, V_{i m p}=-2.0 t$

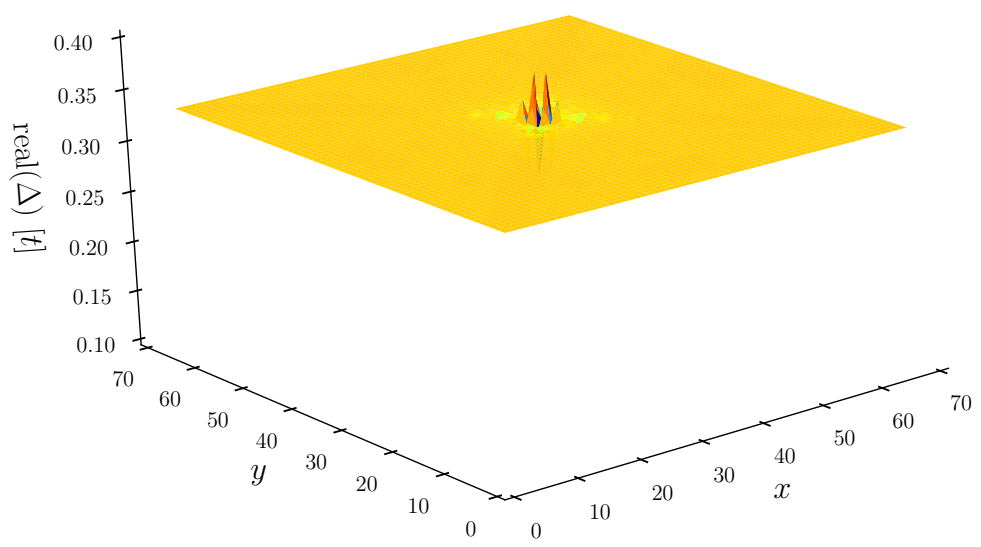

Figure 5.18: Real part of the order parameter for the case of a $64 \times 64$ lattice with $\mu=1.0 t, h=1.5 t$, $\alpha=2.0 t$ and $U=-4.445 t$ corresponding to $\nu=-2$ with the impurity potential $V_{\mathrm{imp}}=-2.0 t$. 
(a)

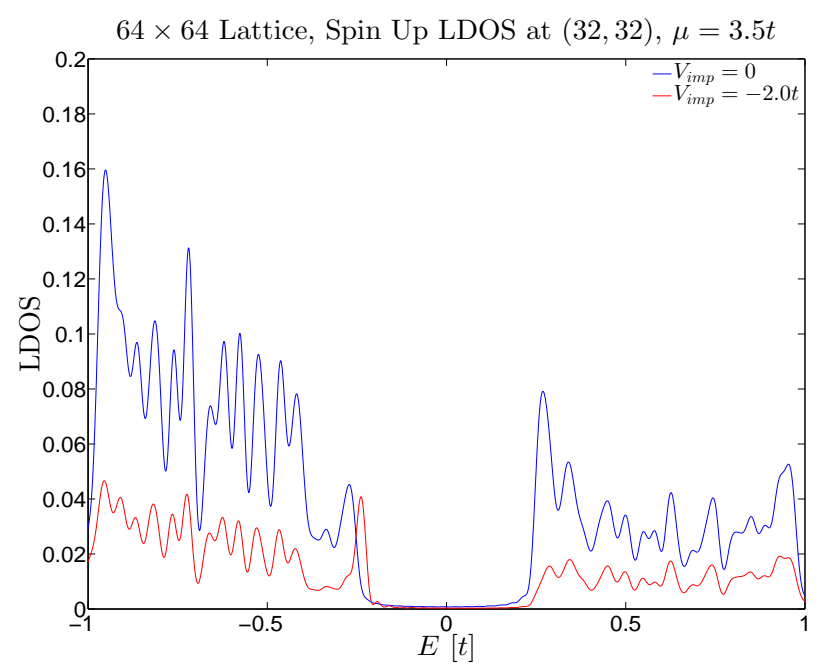

(b)

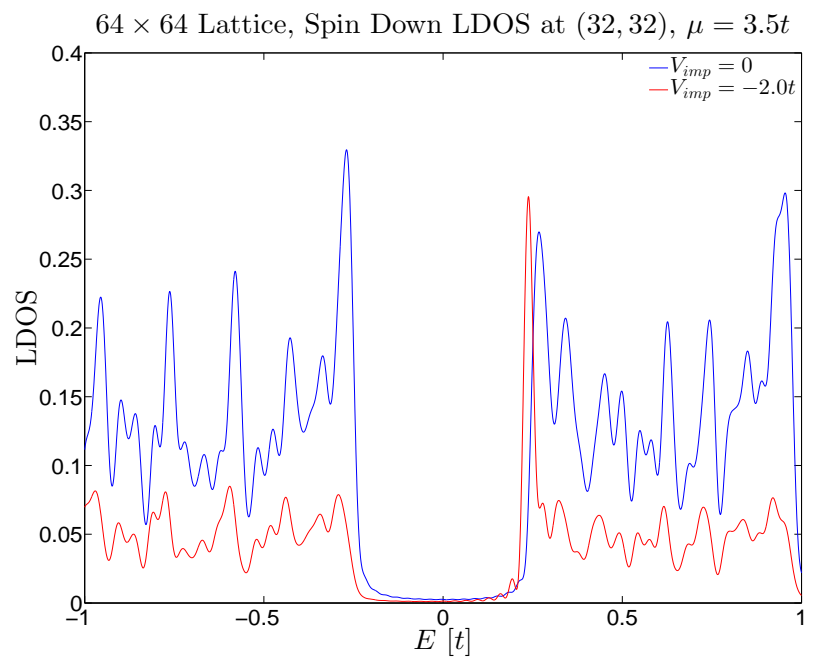

Figure 5.19: (a) Spin-up LDOS for the odd TKNN system $\nu=1$ with $\mu=3.5 t, h=1.0 t, \alpha=1.0 t$. (b) Spin-down LDOS for the system described in (a).

(a)

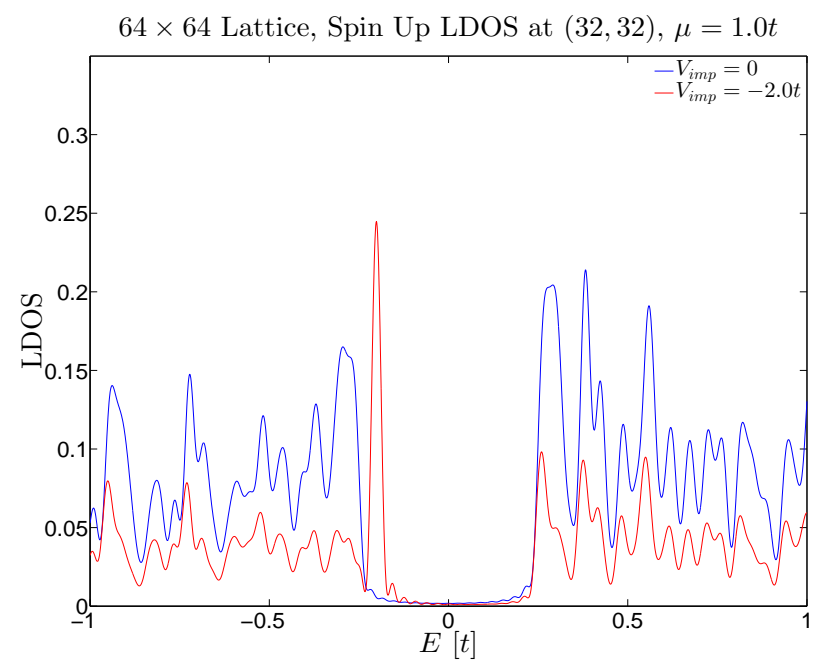

(b)

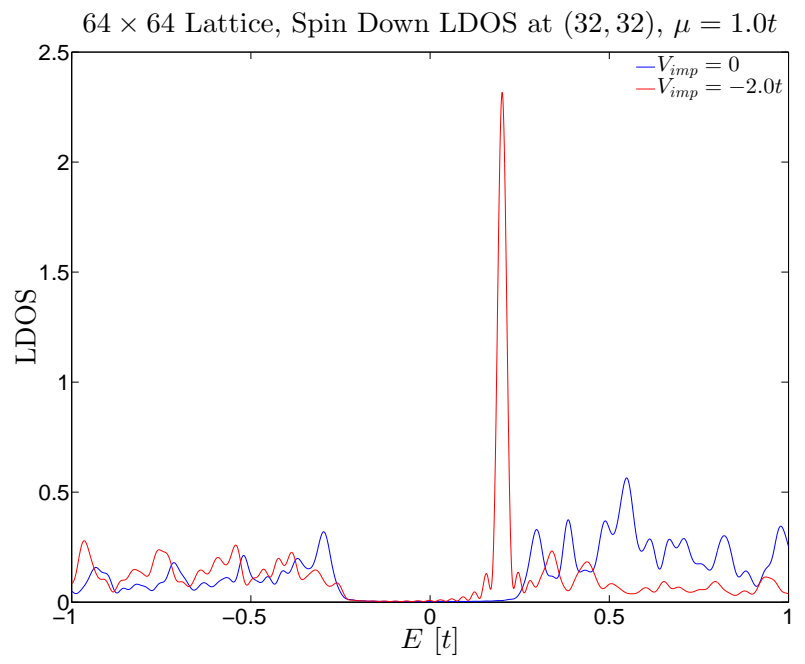

Figure 5.20: (a) Spin-up LDOS for the even TKNN system $\nu=-2$ with $\mu=1.0 t, h=1.5 t, \alpha=2.0 t$. (b) Spin-down LDOS for the system described in (a). In contrast to the odd TKNN case, the presence of impurity bound states are clearly distinguishable in the LDOS. 


\section{CHAPTER 6}

\section{Charge Density Modulations and Topologi- CAL Superconductivity}

In this chapter, we show the existence of topological charge density waves (TCDW) and investigate their interplay with topological superconductivity. Within our self-consistent studies, we have found three degenerate ground states at half filling, a pure TSC state, a pure TCDW state with wavevector $\mathbf{Q}=( \pm \pi, \pm \pi)$, and a state in which TSC and TCDW coexist. We begin by studying the bulk system with $\mathrm{PBC}$ in both the $x$ - and $y$-directions and then move onto the case where open edges are present to confirm the appearance of zeroenergy bound states. Lastly, we study the local effects of depositing a single non-magnetic impurity in the center of a sample which is at half-filling.

\subsection{The Pure Density Wave State}

We begin with a study of the pure topological charge density wave (TCDW) state which is obtained by self-consistently solving for the Hartree potential with the superconducting order parameter set to zero. In this way, the particle and hole amplitudes of a quasiparticle are decoupled and we can obtain a pure TCDW state. For a uniform TCDW state with wavevector $\mathbf{Q}$, we can define the CDW order parameter as

$$
\Delta_{C}=\left\langle c_{\mathbf{k} \uparrow}^{\dagger} c_{\mathbf{k}+\mathbf{Q} \uparrow}\right\rangle=\left\langle c_{\mathbf{k} \downarrow}^{\dagger} c_{\mathbf{k}+\mathbf{Q} \downarrow}\right\rangle .
$$


In momentum space, the mean-field Hamiltonian for the pure TCDW state as shown by Sato et al. [55] is given by

$$
H_{\mathrm{CDW}}=\frac{1}{2} \sum_{\mathbf{k}}\left(c_{\mathbf{k} \uparrow}^{\dagger} c_{\mathbf{k} \downarrow}^{\dagger}-c_{\mathbf{k}+\mathbf{Q} \downarrow}^{\dagger} c_{\mathbf{k}+\mathbf{Q} \uparrow}^{\dagger}\right) \mathcal{H}_{\mathrm{BdG}}(\mathbf{k})\left(\begin{array}{c}
c_{\mathbf{k} \uparrow} \\
c_{\mathbf{k} \downarrow} \\
-c_{\mathbf{k}+\mathbf{Q} \downarrow} \\
c_{\mathbf{k}+\mathbf{Q} \uparrow}
\end{array}\right),
$$

where $\mathcal{H}_{\mathrm{BdG}}(\mathbf{k})$ is the BdG Hamiltonian of $\mathrm{CDW}$ :

$$
\mathcal{H}_{\mathrm{BdG}}(\mathbf{k})=\left(\begin{array}{cc}
\epsilon(\mathbf{k})-h \sigma_{z}+\alpha \mathcal{L}(\mathbf{k}) \cdot \boldsymbol{\sigma} & i \Delta_{C} \sigma_{y} \\
-i \Delta_{C} \sigma_{y} & \epsilon(\mathbf{k}+\mathbf{Q})+h \sigma_{z}-\alpha \mathcal{L}(\mathbf{k}+\mathbf{Q}) \cdot \boldsymbol{\sigma}
\end{array}\right)
$$

with $\epsilon(\mathbf{k})=-2 t\left(\cos k_{x}+\cos k_{y}\right)-\mu$ for a square lattice, and $\alpha \mathcal{L}(\mathbf{k})=\alpha\left(\sin k_{y},-\sin k_{x}\right)$. We note that Eq. (67) of Sato et al. contains a typo in the $(2,2)$ element of the Hamiltonian in which the the spin-orbit term $\alpha \mathcal{L}(\mathbf{k}+\mathbf{Q})$ is missing a minus sign. As shown in Appendix $\mathrm{C}$, once this typo is taken into account, the BdG Hamiltonian for TCDW (6.3) is equivalent to the BdG Hamiltonian for TSC (5.9) if $\epsilon(\mathbf{k}+\mathbf{Q})=-\epsilon(\mathbf{k})$ and $\mathcal{L}(\mathbf{k}+\mathbf{Q})=-\mathcal{L}(\mathbf{k})$. These conditions are satisfied if we take $\mathbf{Q}=( \pm \pi, \pm \pi)$ for the CDW states we have found at halffilling $(\mu=0)$ where there is one electron per site on average. Therefore, our CDW states have the topological regions as classified by Table 5.1 [55] with $\mu$ replaced by $\tilde{\mu}$ and $h$ replaced by $\tilde{h}$. Moreover, the third condition in region (b) of Table 5.1 disappears for $\mu=0$, implying that there can only exist trivial and Abelian topological phases for the results presented in this chapter.

First, we take a $50 \times 50$ lattice with surfaces present at $x=0$ and $x=49$ and parameters $\mu=0, h=1.5 t, \alpha=2.5 t$ and $\Delta_{C}=1.0 t$. The energy spectrum as a function of $k_{y}$ is obtained and illustrated in Fig. 6.1. In agreement with the fact that the system is in the Abelian topological phase, we observe gapless edge modes at $k_{y}=0$ and $k_{y}=\pi$ (equivalent to $-\pi)$. Since we are in the Abelian phase, the in-gap zero-energy states are not Majorana fermions but are instead Dirac fermions and hence the edge states obtained will be analogous to those in a topological insulator. Moreover, as the CDW effectively doubles the lattice 


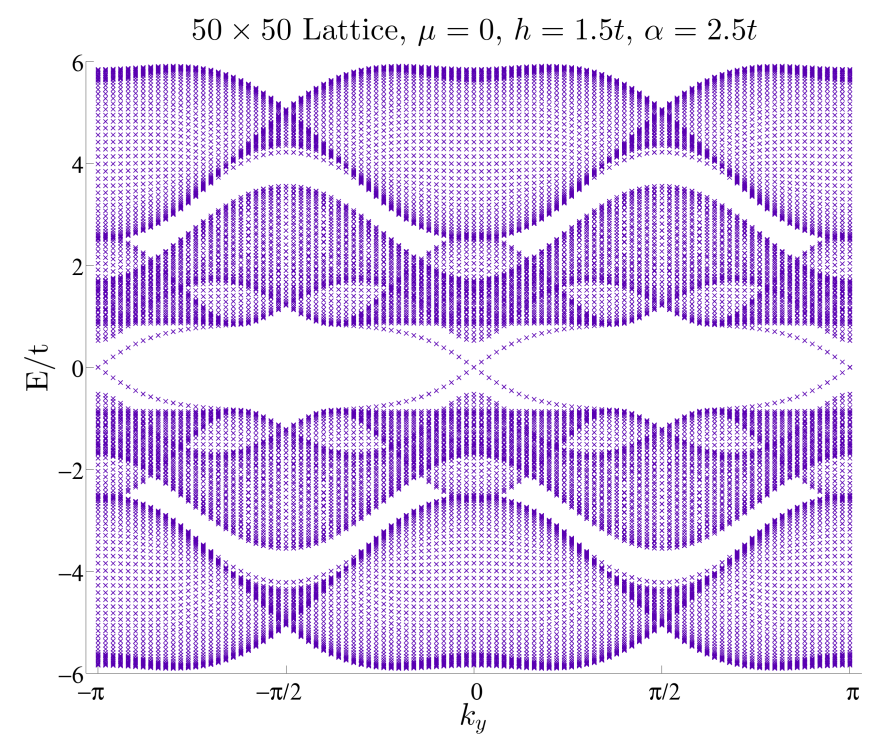

Figure 6.1: Energy spectra for a $50 \times 50$ system in the CDW state with edges at $x=0$ and $x=49$ with parameters $\mu=0, h=1.5 t, \alpha=2.5 t$ and $\Delta_{C}=1.0 t$.

constant from $a$ to $2 a$ in both the $x$ - and $y$-directions, the Brillioun zone is folded in half, meaning that the zero-energy mode at $k_{y}=\pi$ is equivalent to the zero mode at $k_{y}=0$. Therefore, although Fig. 6.1 shows three zero-energy modes, there is in fact only one distinct zero-energy mode for such a system. Despite the odd number of zero modes, however, the system remains in the Abelian topological phase.

As a self-consistent example, we present a $64 \times 64$ system in the pure TCDW state with $\tilde{\mu}=0, h=1.5 t, \alpha=1.0 t, U=-4.0 t$ and PBC. Recall that $\tilde{\mu}$ is the chemical potential measured from the average Hartree potential, $\tilde{\mu}=\mu-\left(\bar{V}_{\uparrow}^{(H)}+\bar{V}_{\downarrow}^{(H)}\right) / 2$. To obtain the CDW order parameter, we solve each component of the Hartree potential self-consistently and obtain a value of $\Delta_{C}=0.67504 t$. With the converged average Hartree potential values for each spin, $\bar{V}_{\uparrow}^{(H)}$ and $\bar{V}_{\downarrow}^{(H)}$, we also have a shifted Zeeman field of $\tilde{h}=0.96135 t$. The up- and down-spin electron densities are shown in Fig. 6.2, both clearly exhibiting periodic density modulations. The checkerboard pattern reflects the fact that the TCDW state has wavevector $\mathbf{Q}=( \pm \pi, \pm \pi)$. We also note that the total converged electron density for this system is exactly $n_{e}=1.0 t$. This numerical result confirms the fact that $\mu=0$ in the case of TCDW/TSC in the presence of a Zeeman field and Rashba spin-orbit coupling corresponds to exactly half filling, just as for conventional $s$-wave superconductivity. We have also calculated 
(a)

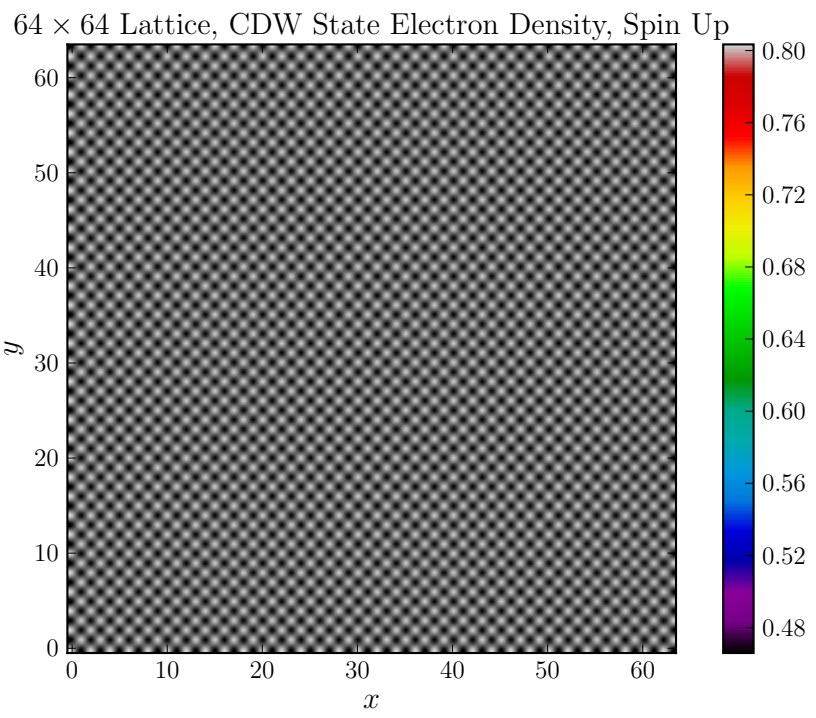

(b)

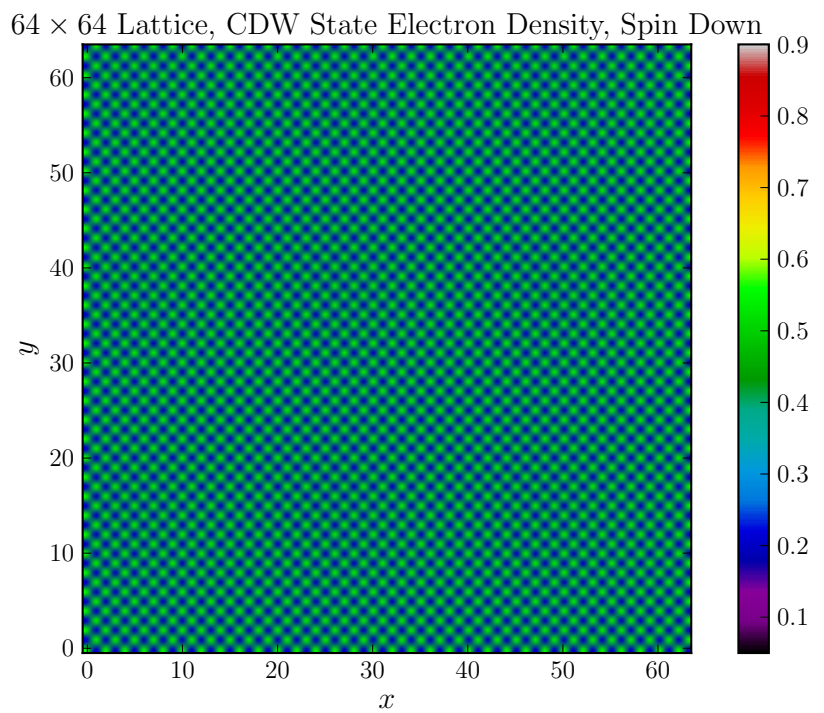

Figure 6.2: (a) Spin up and (b) spin down electron density distributions for an $64 \times 64$ bulk system in the CDW state at half-filling with $h=1.5 t$ and $\alpha=t$.

the ground-state energy of the system given by (B.22),

$$
E_{0}=-\frac{1}{2} \sum_{n}\left(E_{n 1}+E_{n 2}\right)+\frac{1}{2} \sum_{i \sigma}\left(\epsilon_{i \sigma}-\tilde{\mu}+V_{i i \bar{\sigma}}^{(H)}-\bar{V}_{\bar{\sigma}}^{(H)}\right)-\sum_{i} \frac{1}{U_{i i}}\left[V_{i i \uparrow}^{(H)} V_{i i \downarrow}^{(H)}+\left|\Delta_{i i}^{\downarrow \uparrow}\right|^{2}\right]
$$

where $E_{n 1}$ and $E_{n 2}$ are the spin-up and spin-down eigenvalues. Performing the computation, we obtain a ground-state energy of $E_{0}=-8432.72 t$ for the pure CDW state. The spin-up and spin-down local density of states (LDOS) was also computed for sites $(0,0),(0,1),(1,0)$ and $(1,1)$ and is illustrated in Fig 6.3. The LDOS alternates spatially from one site to its nearest-neighbour site across the lattice for both spin up and spin down following the charge density wave pattern.

Zero-energy modes will appear in the system when we introduce open edges in the lattice. To see the appearance of these modes, we increase the system size to $80 \times 80$ and in addition, increase the spin-orbit coupling in order to make the system more stable. This leaves us with parameters $\tilde{\mu}=0, h=1.5 t, \alpha=2.5 t$ and coupling constant $U=-4.0 t$ in the pure CDW state with edges at $x=0$ and $x=79$. Self-consistently solving for the Hartree potential, we obtain a shifted Zeeman field of $\tilde{h}=0.95521 t$. Figure 6.4 shows a cross section of the electron density for $y=40$ and $y=41$ for both spin up and spin down. The center of the 
(a)

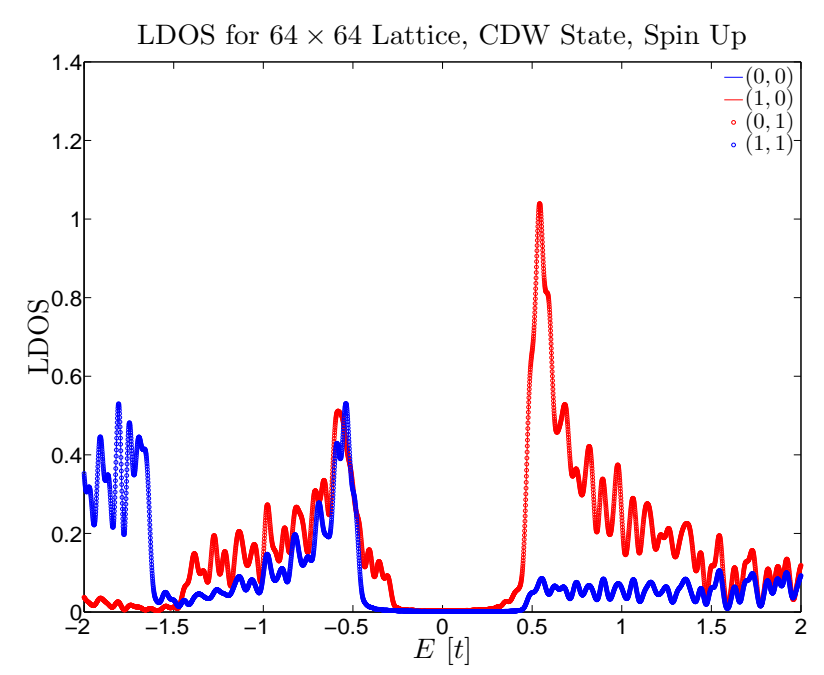

(b)

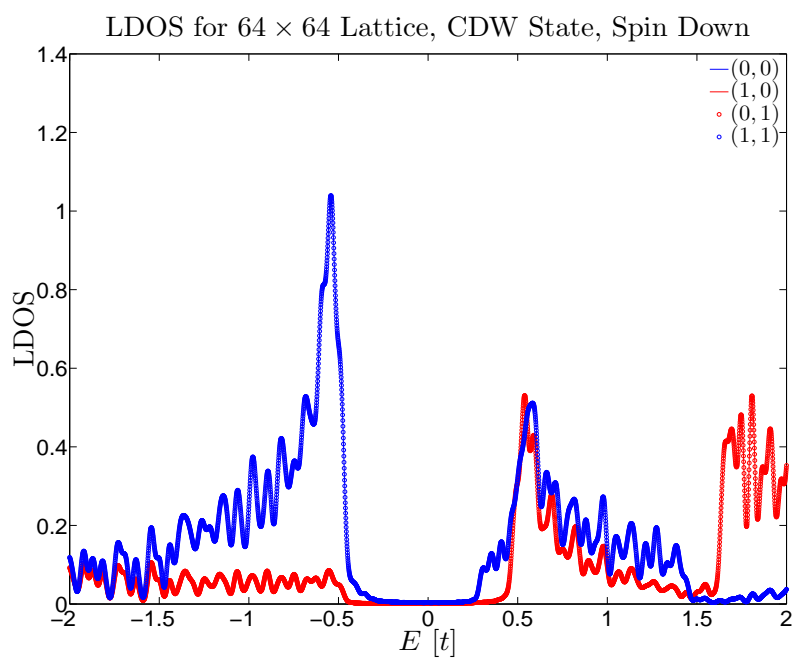

Figure 6.3: (a) Spin-up and (b) spin-down local density of states at sites $(0,0),(0,1),(1,0)$ and $(1,1)$ for a $64 \times 64$ lattice in the CDW state with $\mathrm{PBC}, \tilde{\mu}=0, h=1.5 t$ and $\alpha=1.0 t$. The density modulations in Fig. 6.2 are reflected in the LDOS causing the LDOS to spatially alternate from site to site.

(a)

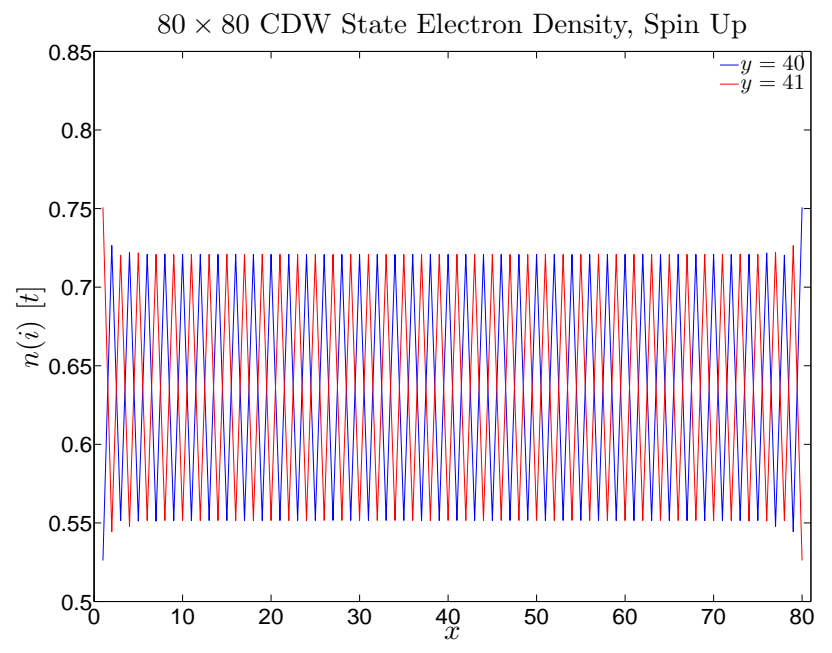

(b)

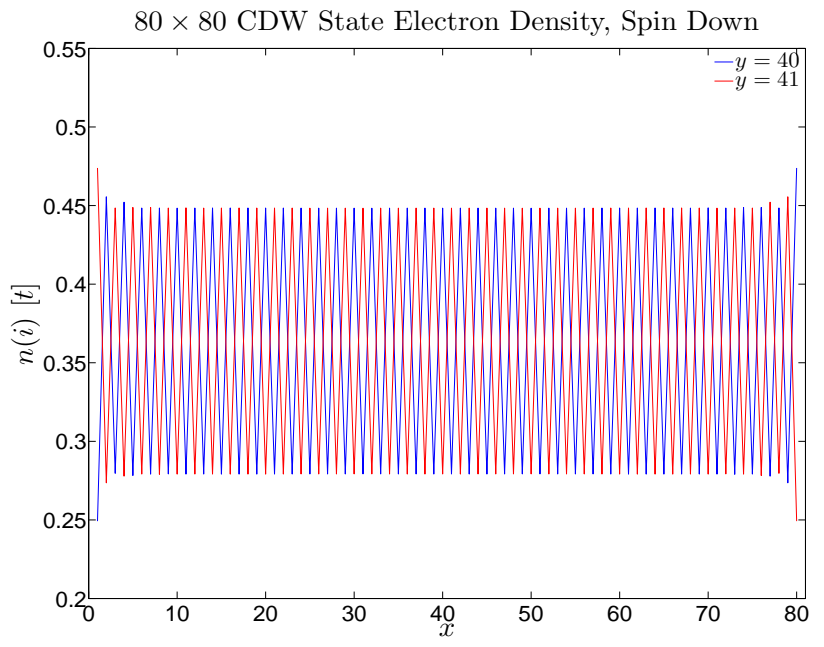

Figure 6.4: (a) Spin-up and (b) spin-down cross section of the electron density distribution at $y=40$ and $y=41$ for an $80 \times 80$ system in the topological CDW state with $\tilde{\mu}=0, h=1.5 t, \alpha=2.5 t$ and coupling constant $U=-4.0 t$.

lattice is largely unaffected by the presence of edges and we observe a perfect $( \pm \pi, \pm \pi)$ CDW in the bulk of the system, while the effects of the open surfaces can be seen in the CDW amplitude for the sites very near $x=0$ and $x=79$. Furthermore, we find that the presence of edges in the system results in the appearance of zero-energy bound states; two zero-energy 
modes on each edge in fact, as the system is in the Abelian topological phase with even

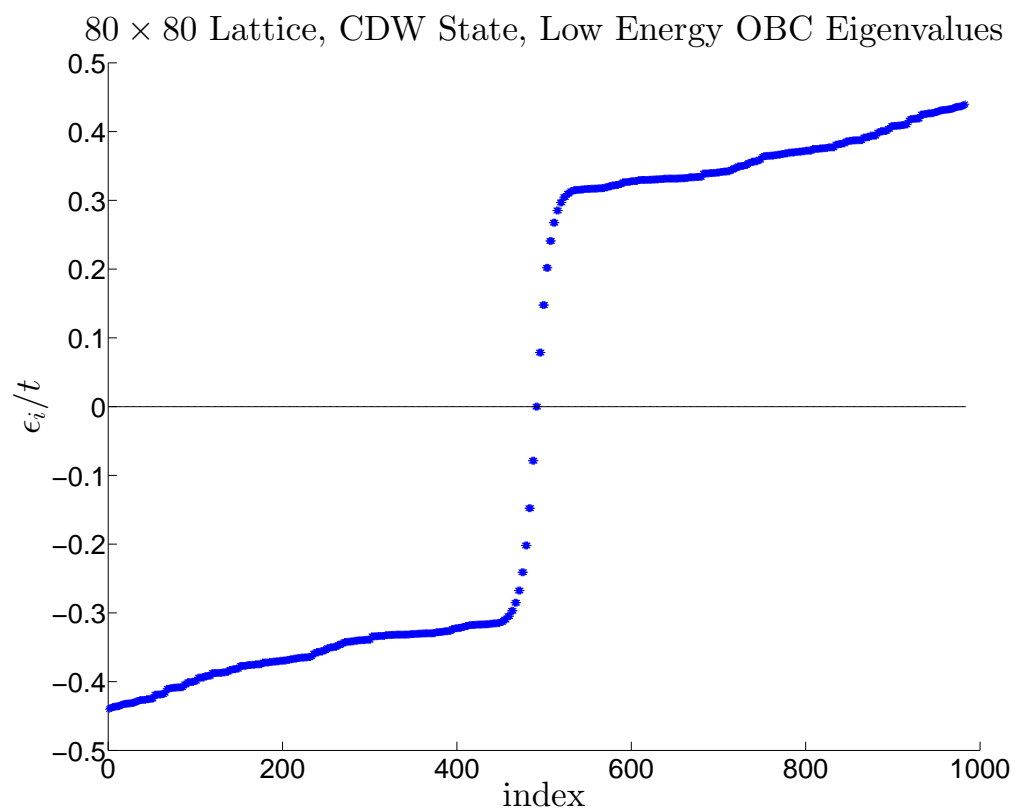

Figure 6.5: Low-energy eigenvalues for Hamiltonian (6.3) for the system described in Fig. 6.4. The presence of edges causes the appearance of in-gap states in the TCDW state just as in the TSC state.

TKNN number. A plot of the eigenvalues vs. index is shown in Fig. 6.5 with the absolute lowest energy eigenvalue being $E=1.23074 \times 10^{-5}$.

\subsection{The Superconducting State}

The Abelian pure superconducting state is obtained just as in the previous chapter using the BdG Hamiltonian (5.9) and including the superconducting order parameter. Starting with the same bulk-system parameters as in the previous section, $\tilde{\mu}=0, h=1.5 t, \alpha=1.0 t$ and $U=-4.0 t$, we take a $64 \times 64$ lattice and self-consistently solve for the superconducting order parameter and the Hartree potential. Once self-consistency has been achieved, we obtain an order parameter of $\Delta_{S}=0.67504 t$ and uniform spin-up and spin-down electron densities of $n_{\mathrm{up}}=0.63466 t$ and $n_{\text {down }}=0.36534 t$, respectively, giving a total electron density of $n_{e}=1.0 t$, as expected. First, we notice that the SC order parameter obtained is identical to the CDW order parameter obtained in the previous section. Furthermore, we obtain the ground-state energy $E_{0}=-8432.72 t$ and a shifted Zeeman field of $\tilde{h}=0.96135 t$-identical to the values 
obtained in the pure TCDW state. Hence we find that just as in the conventional $s$-wave case [83], the superconducting state with uniform density distribution and the CDW state with modulating electron density are degenerate at half-filling. The site-averaged density of states for both spin up and spin down for this pure TSC state is presented in Fig. 6.6. Contrary to the LDOS for the CDW state which alternates from site to site, the uniform spin-up and spin-down charge density results in the local density of states at each site being identical to the site-averaged density of states for each spin component.

When we introduce surfaces at $x=0$ and $x=79$ once again for parameters $\tilde{\mu}=0$, $h=1.5 t, \alpha=2.5 t$ and $U=-4 t$, two Majorana zero modes appear on each edge. We also obtain a lowest absolute eigenvalue of $E=1.23074 \times 10^{-5}$ and shifted Zeeman field of $\tilde{h}=0.95521 t$ resulting in the same values obtained in the pure CDW state. In this case, we observe significant enhancement in the superconducting order parameter at $x=0$ and $x=79$ caused by the presence of edges as can be seen in Fig. 6.7.

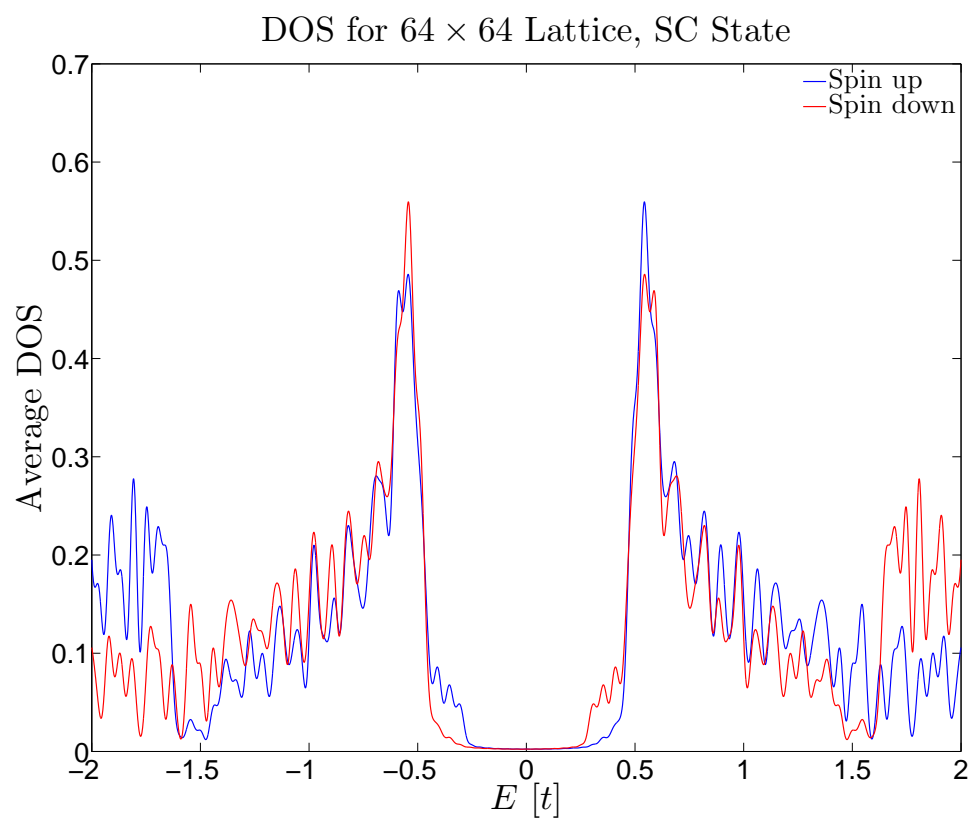

Figure 6.6: Spin-up and spin-down site-averaged density of states for an $64 \times 64$ bulk superconducting system with $\tilde{\mu}=0, h=1.5 t$ and $\alpha=1.0 t$. 


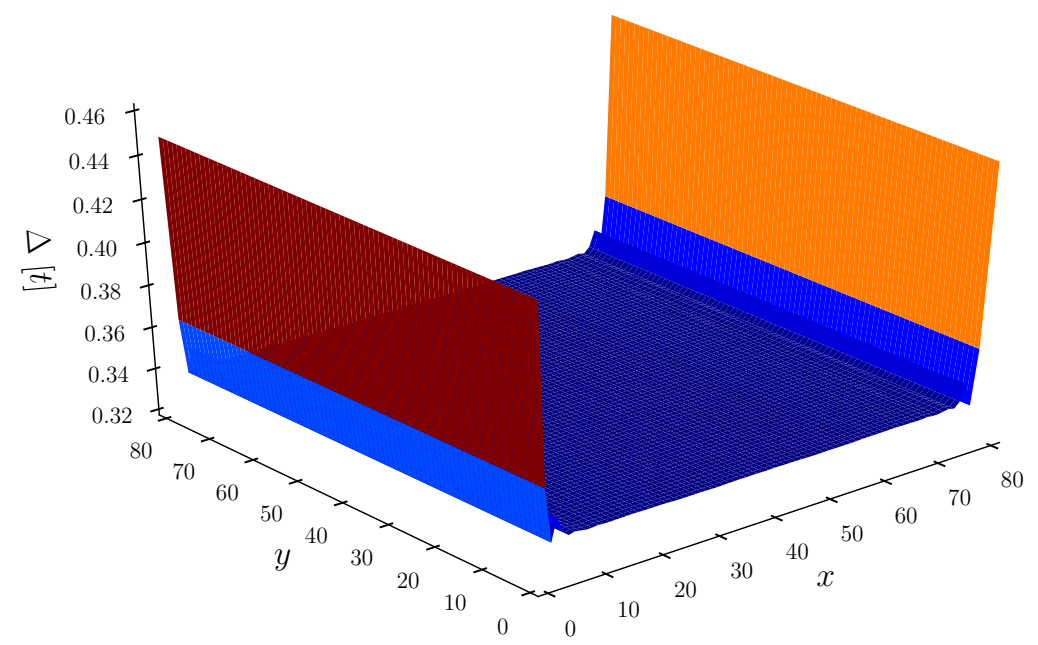

Figure 6.7: Superconducting order parameter for the pure TSC state with $\tilde{\mu}=0, h=1.5 t, \alpha=2.5 t$, $U=-4.0 t$ and edges at $x=0$ and $x=79$.

\subsection{The Mixed Superconducting Density Wave State}

So far, we have found both a TCDW state with periodic density modulations and a TSC state with uniform charge density, each with the same order parameter and ground-state energy. Now we show that these states can in fact coexist with one another. Consider a $64 \times 64$ bulk system with $\tilde{\mu}=0, h=1.5 t, \alpha=1.0 t$ and $U=-4.0 t$. We begin our selfconsistent iterations with the system initially having both a constant superconducting order parameter and periodic density modulations with wavevector $\mathbf{Q}=( \pm \pi, \pm \pi)$. In this way, we are able to obtain converged, self-consistent solutions for the Abelian mixed TSC and TCDW state. After convergence, order parameter values of $\Delta_{S}=0.42169 t$ and $\Delta_{C}=0.52712 t$ were obtained. In this mixed state, the CDW order parameter is suppressed to $78 \%$ of its value in the pure state, while the SC order parameter becomes suppressed to $62 \%$ of its pure state value. A ground-state energy of $E_{0}=-8432.72 t$ and a shifted Zeeman field of $\tilde{h}=0.96135 t$ were obtained for this state and hence we have found three degenerate ground states: a pure TCDW state, a pure TSC state and a mixed TSC+TCDW state. A summary of the values for all three of these Abelian topological states are presented in Table 6.1. 
(a)

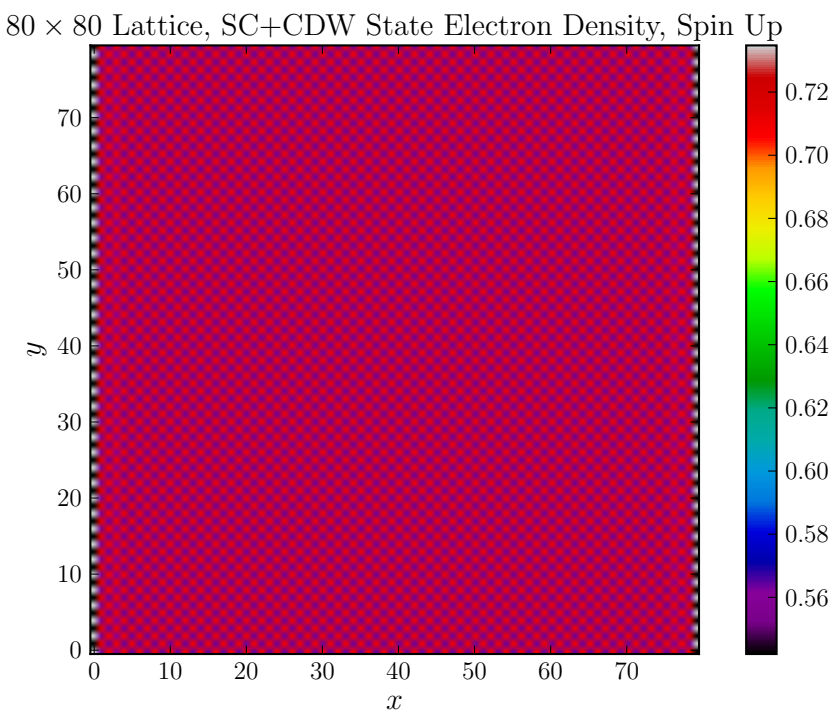

(b)

$80 \times 80$ Lattice, SC+CDW State Electron Density, Spin Down

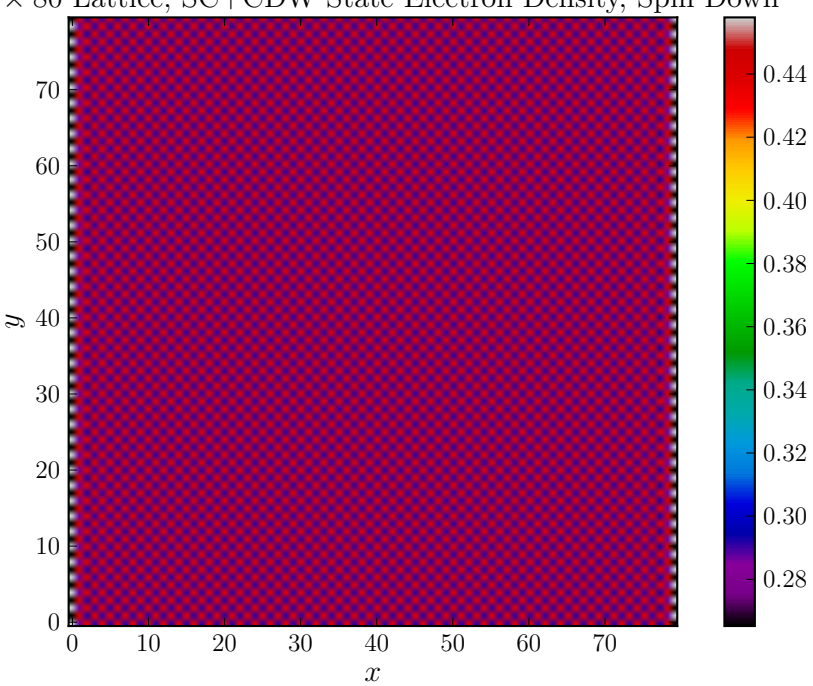

Figure 6.8: (a) Spin-up and (b) spin-down electron density distributions for an $80 \times 80$ lattice in the topological SC+CDW state at half-filling with $h=1.5 t$ and $\alpha=2.5 t$. The presence of edges at $x=0$ and $x=79$ causes two zero-energy Majorana modes to appear at each edge.

\begin{tabular}{cccc}
\hline State & $\Delta_{S}[t]$ & $\Delta_{C}[t]$ & $E_{0}[t]$ \\
\hline SC & 0.67504 & 0.00000 & -8432.72 \\
CDW & 0.00000 & 0.67504 & -8432.72 \\
SC+CDW & 0.42169 & 0.52712 & -8432.72 \\
\hline
\end{tabular}

Table 6.1: Results of the converged order parameters and ground-state energies for each of the three TSC, TCDW and TSC+TCDW states. Just as in the case of conventional $s$-wave superconductivity, each of the three states are degenerate with the same ground-state energy.

Lastly, for the mixed state with parameters $\tilde{\mu}=0, h=1.5 t, \alpha=2.5 t, U=-4.0 t$ and edges at $x=0$ and $x=79$, we again find that the shifted Zeeman field is $\tilde{h}=0.95521 t$ and the lowest absolute eigenvalue for the system is $E=1.23074 \times 10^{-5}$, with two Majorana zero modes being localized on each edge. A surface plot of the spin-up and spin-down electron densities for the system is shown in Fig 6.8. Being able to observe the entire lattice allows us to see how the surface effects are really confined to the very edge of the material and we observe a perfect $( \pm \pi, \pm \pi) \mathrm{CDW}$ in the bulk. The SC order parameter for the system has a structure identical to that of Fig. 6.7; however, the bulk value is reduced to $\Delta_{S \text {,bulk }} \simeq 0.174 t$. 


\subsubsection{Impurity Effects}

Lastly, we study the effects of a single non-magnetic impurity at the center of a topological superconductor at half-filling, this time also solving for the Hartree potential self consistently. To this end, we first deposit a single attractive impurity potential of $V_{\text {imp }}=-1.0 t$ on a $51 \times 51$ lattice at site $(25,25)$ with parameters $\tilde{\mu}=0, h=1.5 t, \alpha=2.5 t, U=-4.0 t$ and PBC. We illustrate the converged spin-up and spin-down electron densities in Fig. 6.9 as well as the converged superconducting order parameter in Fig. 6.10. Although no charge density wave has been inputted into the system, we see that upon depositing a single nonmagnetic impurity in the system, we obtain periodic modulations in the electron density with periodicity $( \pm \pi, \pm \pi)$. Just as in the conventional $s$-wave case, we see that an impurity pins the phase of the charge density oscillations stabilizing a CDW [83]. In the clean system, the TKNN number for a system with $\mu=0, h=1.5 t, \alpha=2.5 t$, and $U=-4.0 t$ is even as mentioned in the previous sections. Examining the order parameter presented in Fig. 6.10 with the impurity present, we observe that the impurity has quite a large effect on the system, extending out to approximately 20 lattice sites away from the impurity site $(25,25)$. This result is in agreement with the results presented in Section 5.1.3 where the effects of a single non-magnetic impurity in the context of both Abelian and non-Abelian TSC were examined. 
(a)

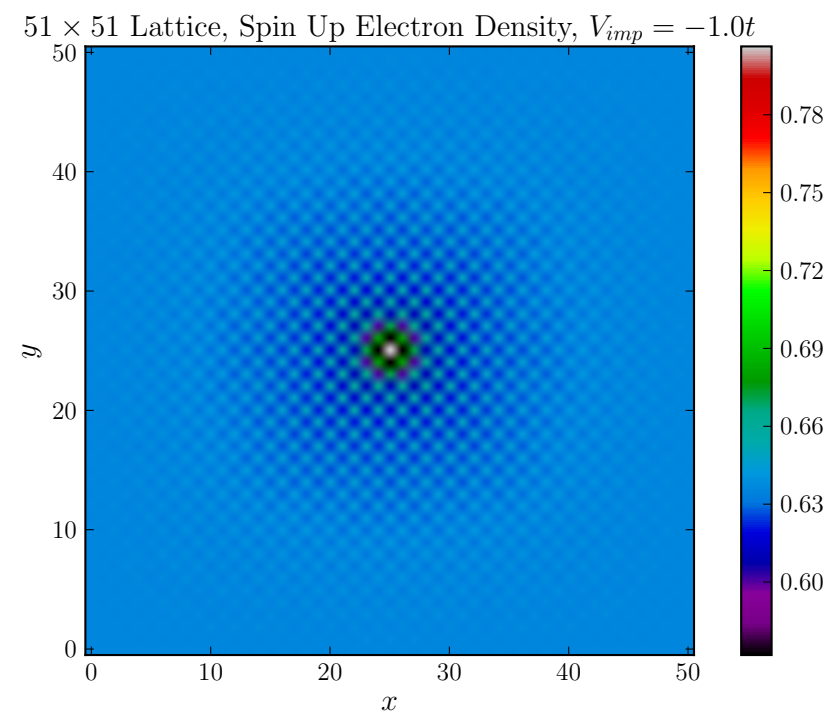

(b)

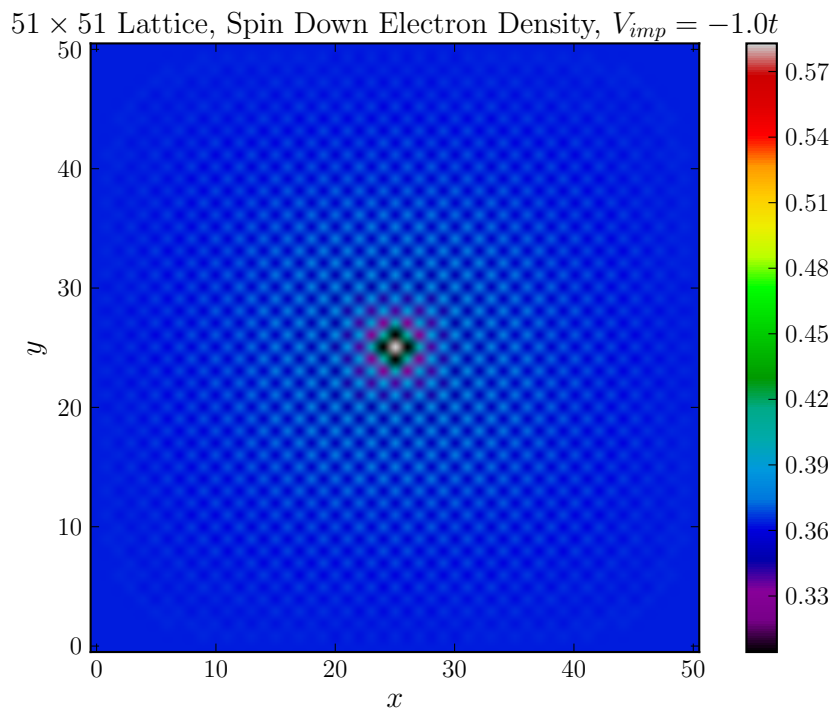

Figure 6.9: (a) Spin-up and (b) spin-down electron density distributions for a $51 \times 51$ lattice with $\tilde{\mu}=0, h=1.5 t, \alpha=2.5 t, U=-4.0 t$ and PBC. In the center of the lattice, we have placed a single impurity potential $V_{\mathrm{imp}}=-1.0 t$ which results in the appearance of CDW.

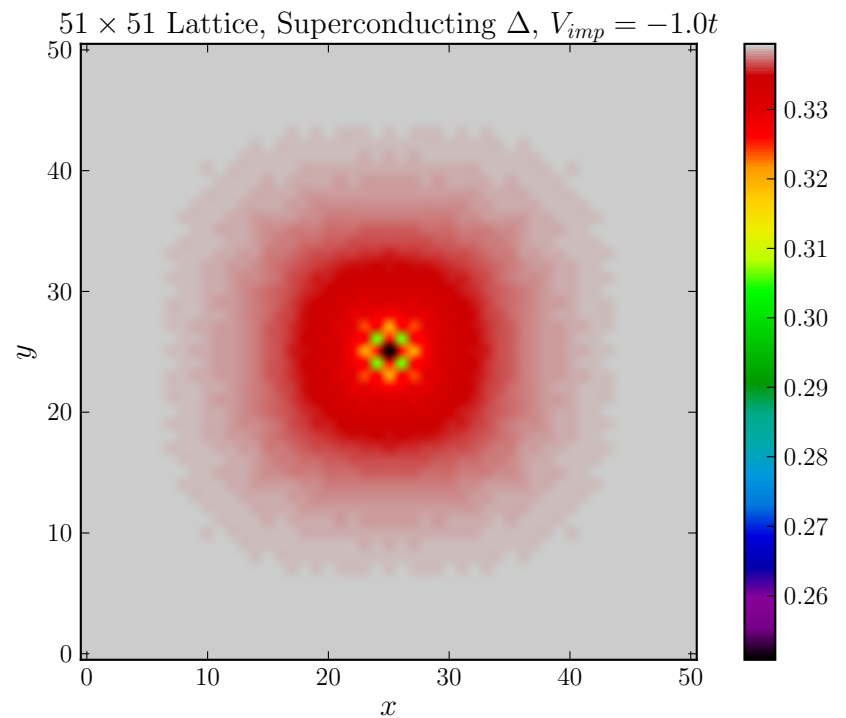

Figure 6.10: Superconducting order parameter for the $51 \times 51$ system presented in Fig. 6.9 with $V_{\text {imp }}=-1.0 t$ at site $(25,25)$. We observe impurity effects in the order parameter quite far from the impurity site consistent with the even TKNN number results presented in Section 5.1.3. 


\section{Chapter 7}

\section{Conclusion}

In this thesis, we have performed microscopic mean-field calculations within a twodimensional (2D) model for topological superconductivity (TSC) and obtained converged solutions for the order parameter and the Hartree potential. As the Bogoliuobov-de Gennes $(\mathrm{BdG})$ equations for topological superconductivity have extremely high numerical demand, we have implemented the method of Chebyshev polynomial expansion, which allows for self-consistent determination of the mean fields without any direct diagonalization of the Hamiltonian by expanding the spectral density of the Green function with a set of Chebyshev polynomials [47]. To obtain eigenpairs of the converged systems, the recently developed Sakurai-Sugiura method was also implemented which reduces the size of the Hamiltonian in order to obtain eigenvalues within a desired energy range [49]. Calculations were performed on a wide variety of Westgrid computing clusters.

In Chapter 5 the relation between Abelian, non-Abelian and trivial topological order was examined in relation to gapless edge states and the TKNN number. We have illustrated that if the TKNN number is zero, no gapless edge states appear and the system is in the trivial phase. Abelian and non-Abelian topological order corresponds to even and odd TKNN numbers and also to an even and odd number of gapless edge states, respectively. Performing self-consistent calculations, we examined how the electron-phonon coupling constant should be chosen so that the desired topological phase is obtained after self-consistency is achieved. Furthermore, we illustrated how self-consistently solving for the Hartree potential alters the topological regions found in Ref. [55]. Due to the effective reduction of the Zeeman field that occurs when the Hartree potential is included, one must in general choose a much lower coupling constant than in the case where only the order parameter is solved self-consistently. When edges were present in the system, we have observed zero-energy Majorana fermions 
localized at these edges provided that the spectral gap is sufficiently large.

We have also analyzed the critical temperature of the system for both even and odd values of the TKNN number. In this work, we were motivated by a momentum-space study of impurity effects in an $s$-wave topological superconductor performed by Nagai, Ota and Machida [1]. First, we provided revised values of the low-temperature superconducting order parameters demonstrating the power of the BdG theory. Equating the zero-temperature order parameters, we then examined the non-Abelian system in both a low and high Zeeman field region, finding that a large Zeeman field has detrimental effects on the critical temperature of the system due to the spin being well polarized in agreement with [1]. We also found that the even TKNN system was slightly more robust to temperature than its odd TKNN counterpart, owing perhaps to the relatively large spin-orbit coupling. Future work could perhaps examine the differences in impurity effects in the lower Rashba region for both the Abelian and non-Abelian phases. We have then investigated the effects of a single non-magnetic impurity potential placed at the center of the lattice for two of the systems considered within our temperature study. Despite the relatively weak impurity potential, we found a large suppression of the order parameter at the impurity site in the non-Abelian case indicating unconventional superconducting behaviour. In the Abelian topological phase, the behaviour is more reminiscent of conventional superconductivity than its non-Abelian counterpart, however, we observed oscillations in the order parameter around the impurity, extending out quite far from the impurity site. This lead to the observation of a pronounced impurity bound state well within the gap, in addition to a second impurity bound state near the gap edge. Hence we conclude that the non-Abelian topological phase in our $s$-wave model shows signs of unconventional $p$-wave behaviour, as predicted by Sato et al. [55]. While the Abelian topological phase did not exhibit the same features of unconventional superconductivity as its non-Abelian counterpart, the Abelian phase still presented its own interesting behaviour owing to its topological nature.

In Chapter 6, by self-consistently solving for the Hartree potential as well as the superconducting order parameter, we have shown the existence of topological charge density waves (TCDW) and have investigated the interplay between TCDW and TSC at half filling. Three degenerate ground states have been found: a pure TCDW state, a pure TSC state and a 
mixed TCDW+TSC state, demonstrating that TCDW and TSC can in fact coexist together at half filling, just as conventional $s$-wave SC and CDW can coexist [83]. Upon imposing open surfaces, we then found zero-energy modes in each of the three states at the same eigenvalue. As calculations were performed at a chemical potential of $\tilde{\mu}=0$, all states were necessarily in the Abelian topological phase with even TKNN number and hence two zero-energy modes were localized on each edge. Lastly, by placing a single attractive impurity potential in a system at half filling with no input charge density wave, we found that just as in the conventional $s$-wave case, the impurity pins the phase of the charge density oscillations causing a CDW to be stabilized [83]. It was also demonstrated that the impurity potential does not cause significant suppression at the impurity site, in agreement with the Abelian results of Chapter 5 .

In this thesis, we have examined the topological nature of SC and CDW within the 2D TSC model of Sato et al. [55] applied to a minimal tight-binding model. For this purpose, the mean fields were solved self-consistently on a square lattice with nearest-neighbour hopping only. Future work could extend this model to describe different band structures or more complex crystal structures. Although it is straightforward to generalize our current model in this regard, such extension would inevitably require more computational resources. Thus, one must carefully consider what new insight can be gained as to the fundamental nature of TSC and TCDW through such an extension. Future work could also study the possible origin of TCDW in this system, whether it be Fermi-surface nesting, van Hove singularities, or yet another mechanism. While we observed a mixed TSC+TCDW state obtained by placing a single non-magnetic impurity for $\tilde{\mu}=0$, finding mixed states with regular density oscillations for nonzero $\tilde{\mu}$ may be a possibility. In fact, it is known in the case of conventional $s$-wave systems that CDW with the wavevector $( \pm \pi, \pm \pi)$ can exist away from half filling [84]. With nonzero $\tilde{\mu}$, one could explore the existence of TCDW in the non-Abelian phase and the effects of impurities in mixed states for odd TKNN number.

Even in the context of conventional $s$-wave SC, the interplay between SC and CDW remains up for debate. Moreover, the mechanism behind charge density modulations in a wide variety of superconducting materials remains unknown. The self-consistent studies presented in this thesis of TCDW within a 2D TSC model is the first step in the process 
towards understanding the origin of TCDW. It is hoped that the results presented in this thesis provide motivation for further work in the area of TSC and TCDW. 


\section{REFERENCES}

[1] Y. Nagai, Y. Ota, and M. Machida. Impurity effects in a two-dimensional topological superconductor: A link of $\mathrm{T}_{c}$ robustness with a topological number. J. Phys. Soc. Jpn. 83, 094722 (2014).

[2] J. Bardeen, L. N. Cooper, and J. R. Schrieffer. Theory of superconductivity. Phys. Rev. 108, 1175-1204 (1957).

[3] J. G. Bednorz and K. A. Müller. Possible high $\mathrm{T}_{c}$ superconductivity in the Ba-La-Cu-O system. Z. Phys. B Cond. Matt. 64, 189-193 (1986).

[4] A. Schilling, M. Cantoni, J. Guo, and H. Ott. Superconductivity above 130 K in the Hg-Ba-Ca-Cu-O system. Nature 363, 56-58 (1993).

[5] A. Drozdov, M. Eremets, I. Troyan, V. Ksenofontov, and S. Shylin. Conventional superconductivity at 203 kelvin at high pressures in the sulfur hydride system. Nature 525, 73-76 (2015).

[6] D. Thouless, M. Kohmoto, M. Nightingale, and M. den Nijs. Quantized Hall conductance in a two-dimensional periodic potential. Phys. Rev. Lett. 49, 405-408 (1982).

[7] M. Z. Hasan and C. L. Kane. Colloquium: Topological insulators. Rev. Mod. Phys. 82, 3045-3066 (2010).

[8] B. A. Bernevig. Topological insulators and topological superconductors. Princeton University Press (2013).

[9] S. C. Zhang. Viewpoint: Topological states of quantum matter. Physics 1, 6 (2008).

[10] C. L. Kane and E. J. Mele. $\mathrm{Z}_{2}$ topological order and the quantum spin Hall effect. Phys. Rev. Lett. 95, 146802 (2005).

[11] Kramers H. A. Proc. Amsterdam Acad. 33, 959 (1930).

[12] C. L. Kane and E. J. Mele. Quantum spin Hall effect in graphene. Phys. Rev. Lett. 95, 226801 (2005).

[13] B. A. Bernevig, T. L. Hughes, and S. C. Zhang. Quantum spin Hall effect and topological phase transition in HgTe quantum wells. Science 314, 1757-1761 (2006).

[14] M. König, S. Wiedmann, C. Brne, A. Roth, H. Buhmann, L. W. Molenkamp, X. Qi, and S. Zhang. Quantum spin Hall insulator state in HgTe quantum wells. Science 318, 766-770 (2007). 
[15] D. Hsieh, D. Qian, L. Wray, Y. Xia, Y. S. Hor, R. J. Cava, and M. Z. Hasan. A topological Dirac insulator in a quantum spin Hall phase. Nature 452, 970-974 (2008).

[16] Y. Xia, D. Qian, D. Hsieh, L. Wray, A. Pal, H. Lin, A. Bansil, D. Grauer, Y. S. Hor, R. J. Cava, and M. Z. Hasan. Observation of a large-gap topological-insulator class with a single Dirac cone on the surface. Nat. Phys. 5, 398-402 (2009).

[17] Y. L. Chen, J. G. Analytis, J. H. Chu, Z. K. Liu, S. K. Mo, X. L. Qi, H. J. Zhang, D. H. Lu, X. Dai, Z. Fang, S. C. Zhang, I. R. Fisher, Z. Hussain, and Z. X. Shen. Experimental realization of a three-dimensional topological insulator, $\mathrm{Bi}_{2} \mathrm{Te}_{3}$. Science 325, 178-181 (2009).

[18] D. Hsieh, Y. Xia, D. Qian, L. Wray, F. Meier, J. H. Dil, J. Osterwalder, L. Patthey, A. V. Fedorov, H. Lin, A. Bansil, D. Grauer, Y. S. Hor, R. J. Cava, and M. Z. Hasan. Observation of time-reversal-protected single-Dirac-cone topological-insulator states in $\mathrm{Bi}_{2} \mathrm{Te}_{3}$ and $\mathrm{Sb}_{2} \mathrm{Te}_{3}$. Phys. Rev. Lett. 103, 146401 (2009).

[19] Y. Jiang, Y. Wang, M. Chen, Z. Li, C. Song, K. He, L. Wang, X. Chen, X. Ma, and Q. K. Xue. Landau quantization and the thickness limit of topological insulator thin films of $\mathrm{Sb}_{2} \mathrm{Te}_{3}$. Phys. Rev. Lett. 108, 016401 (2012).

[20] Su-Yang Xu, L. A. Wray, Y. Xia, R. Shankar, A. Petersen, A. Fedorov, H. Lin, A. Bansil, Y. S. Hor, D. Grauer, et al. Discovery of several large families of topological insulator classes with backscattering-suppressed spin-polarized single-Dirac-cone on the surface. arXiv:1007.5111 (2010).

[21] Z. Ren, A. A. Taskin, S. Sasaki, K. Segawa, and Y. Ando. Large bulk resistivity and surface quantum oscillations in the topological insulator $\mathrm{Bi}_{2} \mathrm{Te}_{2} \mathrm{Se}$. Phys. Rev. B 82, 241306 (2010).

[22] T. Sato, K. Segawa, H. Guo, K. Sugawara, S. Souma, T. Takahashi, and Y. Ando. Direct evidence for the Dirac-cone topological surface states in the ternary chalcogenide TlBiSe $e_{2}$. Phys. Rev. Lett. 105, 136802 (2010).

[23] S. Souma, K. Eto, M. Nomura, K. Nakayama, T. Sato, T. Takahashi, K. Segawa, and Y. Ando. Topological surface states in lead-based ternary telluride $\mathrm{Pb}\left(\mathrm{Bi}_{1-x} \mathrm{Sb}_{x}\right)_{2} \mathrm{Te}_{4}$. Phys. Rev. Lett. 108, 116801 (2012).

[24] N. Read and D. Green. Paired states of fermions in two dimensions with breaking of parity and time-reversal symmetries and the fractional quantum Hall effect. Phys. Rev. B 61, 10267-10297 (2000).

[25] Y. Ando, K. Segawa, S. Sasaki, and M Kriener. Experimental studies of the topological superconductor $\mathrm{Cu}_{x} \mathrm{Bi}_{2} \mathrm{Se}_{3}$. In Journal of Physics: Conference Series volume 449 page 012033. IOP Publishing (2013).

[26] S. Nadj-Perge, I. K. Drozdov, J. Li, H. Chen, S. Jeon, J. Seo, A. H. MacDonald, B. A. Bernevig, and A. Yazdani. Observation of Majorana fermions in ferromagnetic atomic chains on a superconductor. Science 346, 602-607 (2014). 
[27] V. Mourik, K. Zuo, S. M. Frolov, S. R. Plissard, E. P. A. M. Bakkers, and L. P. Kouwenhoven. Signatures of Majorana fermions in hybrid superconductor-semiconductor nanowire devices. Science 336, 1003-1007 (2012).

[28] S. Sasaki et al. Topological superconductivity in $\mathrm{Cu}_{x} \mathrm{Bi}_{2} \mathrm{Se}_{3}$. Phys. Rev. Lett. 107, 217001 (2011).

[29] C. Nayak, S. H. Simon, A. Stern, M. Freedman, and S. Das Sarma. Non-Abelian anyons and topological quantum computation. Rev. Mod. Phys. 80, 1083-1159 (2008).

[30] A. Kitaev. Unpaired Majorana fermions in quantum wires. (2000). arXiv:condmat/0010440.

[31] K. Ishida, H. Mukuda, Y. Kitaoka, Z. Q. Mao, H. Fukazawa, and Y. Maeno. Ru NMR probe of spin susceptibility in the superconducting state of $\mathrm{Sr}_{2} \mathrm{RuO}_{4}$. Phys. Rev. B 63, 060507 (2001).

[32] A. P. Mackenzie, R. K. W. Haselwimmer, A. W. Tyler, G. G. Lonzarich, Y. Mori, S. Nishizaki, and Y. Maeno. Extremely strong dependence of superconductivity on disorder in $\mathrm{Sr}_{2} \mathrm{RuO}_{4}$. Phys. Rev. Lett. 80, 161-164 (1998).

[33] S. Das Sarma, C. Nayak, and S. Tewari. Proposal to stabilize and detect half-quantum vortices in strontium ruthenate thin films: Non-Abelian braiding statistics of vortices in a $p_{x}+i p_{y}$ superconductor. Phys. Rev. B 73, 220502 (2006).

[34] J. Jang, D. G. Ferguson, V. Vakaryuk, R. Budakian, S. B. Chung, P. M. Goldbart, and Y. Maeno. Observation of half-height magnetization steps in $\mathrm{Sr}_{2} \mathrm{RuO}_{4}$. Science 331, 186-188 (2011).

[35] L. Fu and C. L. Kane. Superconducting proximity effect and Majorana fermions at the surface of a topological insulator. Phys. Rev. Lett. 100, 096407 (2008).

[36] M. Veldhorst, M. Snelder, M. Hoek, T. Gang, V. K. Guduru, X. L. Wang, U. Zeitler, W. G. van der Wiel, A. A. Golubov, H. Hilgenkamp, et al. Josephson supercurrent through a topological insulator surface state. Nat. Mater. 11, 417-421 (2012).

[37] J. Xu, C. Liu, M. Wang, J. Ge, Z. Liu, X. Yang, Y. Chen, Y. Liu, Z. Xu, C. Gao, D. Qian, F. Zhang, and J. Jia. Artificial topological superconductor by the proximity effect. Phys. Rev. Lett. 112, 217001 (2014).

[38] L. Fu and E. Berg. Odd-parity topological superconductors: Theory and application to $\mathrm{Cu}_{x} \mathrm{Bi}_{2} \mathrm{Se}_{3}$. Phys. Rev. Lett. 105, 097001 (2010).

[39] N. Levy, T. Zhang, J. Ha, F. Sharifi, A. A. Talin, Y. Kuk, and J. A. Stroscio. Experimental evidence for $s$-wave pairing symmetry in superconducting $\mathrm{Cu}_{x} \mathrm{Bi}_{2} \mathrm{Se}_{3}$ single crystals using a scanning tunneling microscope. Phys. Rev. Lett. 110, 117001 (2013).

[40] J. A. Schneeloch, R. D. Zhong, Z. J. Xu, G. D. Gu, and J. M. Tranquada. Dependence of superconductivity in $\mathrm{Cu}_{x} \mathrm{Bi}_{2} \mathrm{Se}_{3}$ on quenching conditions. Phys. Rev. B 91, 144506 (2015). 
[41] M. Kriener, K. Segawa, Z. Ren, S. Sasaki, S. Wada, S. Kuwabata, and Y. Ando. Electrochemical synthesis and superconducting phase diagram of $\mathrm{Cu}_{x} \mathrm{Bi}_{2} \mathrm{Se}_{3}$. Phys. Rev. $B$ 84, 054513 (2011).

[42] B. Bauer, R. M. Lutchyn, M. B. Hastings, and M. Troyer. Effect of thermal fluctuations in topological p-wave superconductors. Phys. Rev. B 87, 014503 (2013).

[43] M. Sato, Y. Takahashi, and S. Fujimoto. Non-Abelian topological order in $s$-wave superfluids of ultracold fermionic atoms. Phys. Rev. Lett. 103, 020401 (2009).

[44] J. K. Chin, D. E. Miller, Y. Liu, C. Stan, W. Setiawan, C. Sanner, K. Xu, and W. Ketterle. Evidence for superfluidity of ultracold fermions in an optical lattice. Nature 443, 961-964 (2006).

[45] T. Zhou, Y. Gao, and Z. D. Wang. Topological quantum phase transitions and edge states in spin-orbital coupled Fermi gases. Sci. Rep. 4, 5218 (2014).

[46] C. Qu, M. Gong, and C. Zhang. Fflo or majorana superfluids: The fate of fermionic cold atoms in spin-orbit coupled optical lattices. arXiv:1304.3926 (2013).

[47] A. Weiße, G. Wellein, A. Alvermann, and H. Fehske. The kernel polynomial method. Rev. Mod. Phys. 78, 275-306 (2006).

[48] Y. Nagai, Y. Ota, and M. Machida. Efficient numerical self-consistent mean-field approach for fermionic many-body systems by polynomial expansion on spectral density. J. Phys. Soc. Jpn. 81, 024710 (2012).

[49] T. Sakurai and H. Sugiura. A projection method for generalized eigenvalue problems using numerical integration. J. Comput. Appl. Math. 159, 119-128 (2003).

[50] Y. Nagai, Y. Shinohara, Y. Futamura, Y. Ota, and T. Sakurai. Numerical construction of a low-energy effective Hamiltonian in a self-consistent Bogoliubov-de Gennes approach of superconductivity. J. Phys. Soc. Jpn. 82, 094701 (2013).

[51] T. Kiss, Y. Yokoya, A. Chainani, S. Shin, T. Hanaguri, M. Nohara, and H. Takagi. Charge-order-maximized momentum-dependent superconductivity. Nat. Phys. 3, 720725 (2007).

[52] S. V. Borisenko, A. A. Kordyuk, V. B. Zabolotnyy, D. S. Inosov, D. Evtushinsky, B. Büchner, A. N. Yaresko, A. Varykhalov, R. Follath, W. Eberhardt, L. Patthey, and H. Berger. Two Energy Gaps and Fermi-Surface "Arcs" in $\mathrm{NbSe}_{2}$. Phys. Rev. Lett. 102, $166402(2009)$.

[53] G. Ghiringhelli, M. Le Tacon, M. Minola, S. Blanco-Canosa, C. Mazzoli, N. B. Brookes, G. M. De Luca, A. Frano, D. G. Hawthorn, F. He, T. Loew, M. Moretti Sala, D. C. Peets, M. Salluzzo, E. Schierle, R. Sutarto, G. A. Sawatzky, E. Weschke, B. Keimer, and L. Braicovich. Long-range incommensurate charge fluctuations in ( $\mathrm{Y}, \mathrm{Nd}) \mathrm{Ba}_{2} \mathrm{Cu}_{3} \mathrm{O}_{6+x}$. Science 337, 821 (2012). 
[54] T. Wu, H. Mayaffre, S. Krämer, M. Horvatic, C. Berthier, W. N. Hardy, R. Liang, D. A. Bonn, and M. Julien. Magnetic-field-induced charge-stripe order in the high-temperature superconductor $\mathrm{YBa}_{2} \mathrm{Cu}_{3} \mathrm{O}_{y}$. Nature 477, 191-194 (2011).

[55] M. Sato, Y. Takahashi, and S. Fujimoto. Non-Abelian topological orders and Majorana fermions in spin-singlet superconductors. Phys. Rev. B 82, 134521 (2010).

[56] M. Scheurer and J. Schmalian. Topological superconductivity and unconventional pairing in oxide interfaces. Nat. Commun. 6 (2015).

[57] L. N. Cooper. Bound electron pairs in a degenerate Fermi gas. Phys. Rev. 104, 11891190 (1956).

[58] J. R. Schrieffer. Theory of Superconductivity. Westview Press (1999).

[59] P. de Gennes. Superconductivity of Metals and Alloys. Westview Press (1999).

[60] J. Hubbard. Electron correlations in narrow energy bands. Proc. R. Soc. Lond. A 276, 238-257 (1963).

[61] J. A. Wilson, F. J. Di Salvo, and S. Mahajan. Charge-density waves in metallic, layered, transition-metal dichalcogenides. Phys. Rev. Lett. 32, 882-885 (1974).

[62] R. Peierls. Quantum Theory of Solids. Clarendon Press (1955).

[63] R. Peierls. More Surprises in Theoretical Physics. Princeton University Press (1991).

[64] M. D. Johannes and I. I. Mazin. Fermi surface nesting and the origin of charge density waves in metals. Phys. Rev. B 77, 165135 (2008).

[65] T. M. Rice and G. K. Scott. New mechanism for a charge-density-wave instability. Phys. Rev. Lett. 35, 120-123 (1975).

[66] J. Sadowski, K. Tanaka, and Y. Nagai. Effects of band structure and electron-phonon coupling on charge density waves. Can. J. Phys. 91, 487-489 (2013).

[67] Q. Han, T. Li, and Z. D. Wang. Pseudogap and Fermi-arc evolution in the phasefluctuation scenario. Phys. Rev. B 82, 052503 (2010).

[68] L. Covaci, F. M. Peeters, and M. Berciu. Efficient numerical approach to inhomogeneous superconductivity: The Chebyshev-Bogoliubov-de Gennes method. Phys. Rev. Lett. 105, 167006 (2010).

[69] W. Kunishima, M. Itoh, and H. Tanaka. A new method to calculate the Green function by polynomial expansion. Prog. Theo. Phys. Suppl. 138, 149-150 (2000).

[70] Y. Nagai, Y. Ota, and M. Machida. Topological s-wave pairing superconductivity with spatial inhomogeneity: Midgap-state appearance and robustness of superconductivity. J. Phys. Soc. Jpn. 84, 034711 (2015). 
[71] K. v. Klitzing, G. Dorda, and M. Pepper. New method for high-accuracy determination of the fine-structure constant based on quantized Hall resistance. Phys. Rev. Lett. 45, 494-497 (1980).

[72] R. B. Laughlin. Quantized Hall conductivity in two dimensions. Phys. Rev. B 23, 5632 (1981).

[73] M. V. Berry. Quantal phase factors accompanying adiabatic changes. Proc. R. Soc. Lond. 392, 45-57 (1984).

[74] M. Nakahara. Geometry, Topology and Physics. Institute of Physics Publishing (2003).

[75] F. D. M. Haldane. Model for a Quantum Hall Effect without Landau Levels: CondensedMatter Realization of the "Parity Anomaly". Proc. R. Soc. Lond. 61, 2015-2018 (1988).

[76] A. Schnyder, S. Ryu, A. Furusaki, and A. Ludwig. Classification of topological insulators and superconductors in three spatial dimensions. Phys. Rev. B 78, 195125 (2008).

[77] A. Altland and M. Zirnbauer. Nonstandard symmetry classes in mesoscopic normalsuperconducting hybrid structures. Phys. Rev. B 55, 1142 (1997).

[78] Y. Hatsugai. Chern number and edge states in the integer quantum Hall effect. Phys. Rev. Lett. 71, 3697-3700 (1993).

[79] C. Xu and J. E. Moore. Stability of the quantum spin Hall effect: Effects of interactions, disorder, and $\mathrm{Z}_{2}$ topology. Phys. Rev. B 73, 045322 (2006).

[80] M. Leijnse and K. Flensberg. Introduction to topological superconductivity and Majorana fermions. Semicon. Sci. Tech. 27, 124003 (2012).

[81] M. Freedman, A. Kitaev, M. Larsen, and Z. Wang. Topological quantum computation. Bull. Am. Math. Soc. 40, 31-38 (2003).

[82] G. Collins. Computing with quantum knots. Scientific American, pages 57-63 (April 2006).

[83] J. Sadowski. Interplay of charge density modulations and superconductivity. Master's thesis University of Saskatchewan (2011).

[84] Y. Nagai, J. W. Sadowski, and K. Tanaka. Van Hove singularity-driven charge density waves in an s-wave superconductor. Unpublished.

[85] Y. Ando. Topological insulator materials. J. Phys. Soc. Jpn. 82, 102001 (2013). 


\section{ApPEndix A}

\section{DeRIVATION OF THE TKNN NumBeR}

In this Appendix we follow the notation of Ando [85] and derive the TKNN number which represents the topological invariant in 2D topological insulators and superconductors with TRS breaking. Consider a low-temperature (not necessarily zero temperature) twodimensional electron system of size $L \times L$ in a periodic potential where the magnetic field $B$ and electric field $E$ are applied in the $\hat{z}$ - and $\hat{y}$-directions, respectively. We wish to compute the Hall conductivity of such a system. To accomplish this, we treat the effect of the electric field as a perturbation potential $V=-e E y$ and compute the perturbed eigenstate $|n\rangle_{E}$ as follows [85]:

$$
|n\rangle_{E}=|n\rangle+\sum_{m \neq n} \frac{\langle m|-e E y| n\rangle}{\left(E_{n}-E_{m}\right)}|m\rangle
$$

Using this perturbed eigenstate, we calculate the expectation value of the current density in the $\hat{x}$-direction

$$
\begin{aligned}
\left\langle j_{x}\right\rangle_{E} & =\sum_{n} \frac{N_{n}}{N}\left\langle\left. n\right|_{E} \frac{e v_{x}}{L^{2}} \mid n\right\rangle_{E} \\
& =\sum_{n} f\left(E_{n}\right)\left\langle\left. n\right|_{E} \frac{e v_{x}}{L^{2}} \mid n\right\rangle_{E}
\end{aligned}
$$

where $N_{n}$ is the number of electrons in state $n, N$ is the total number of electrons, $v_{x}$ is the electron velocity along the $x$-direction and $f$ is the Fermi-Dirac distribution function. Inserting (A.1) into our expectation value yields

$$
\left\langle j_{x}\right\rangle_{E}=\left\langle j_{x}\right\rangle_{E=0}+\frac{1}{L^{2}} \sum_{n} f\left(E_{n}\right) \sum_{m \neq n}\left(\frac{\left\langle n\left|e v_{x}\right| m\right\rangle\langle m|-e E y| n\rangle}{\left(E_{n}-E_{m}\right)}+\frac{\langle n|-e E y| m\rangle\left\langle m\left|e v_{x}\right| n\right\rangle}{\left(E_{n}-E_{m}\right)}\right) .
$$

Here, $\left\langle j_{x}\right\rangle_{E=0}=\frac{1}{L^{2}} \sum_{n} f\left(E_{n}\right)\left\langle n\left|e v_{x}\right| n\right\rangle$ and we have neglected the second order term in the computation of (A.3).

Next, we make use of the Heisenberg equation of motion given by

$$
\frac{d y}{d t}=v_{y}=\frac{1}{i \hbar}[y, H],
$$


which implies

$$
\begin{aligned}
\left\langle m\left|v_{y}\right| n\right\rangle & =\frac{1}{i \hbar}(\langle m|y H| n\rangle-\langle m|H y| n\rangle) \\
& =\frac{1}{i \hbar}\left(E_{n}-E_{m}\right)\langle m|y| n\rangle
\end{aligned}
$$

Using this result and dropping $\left\langle j_{x}\right\rangle_{E=0}$ as it is not linear in $E$, we can now evaluate the Hall conductivity, $\sigma_{x y}$ given by

$$
\begin{aligned}
\sigma_{x y} & =\frac{\left\langle j_{x}\right\rangle_{E}}{E} \\
& =-\frac{e^{2}}{L^{2}} \sum_{n} f\left(E_{n}\right) \sum_{m \neq n} i \hbar\left(\frac{\left\langle n\left|v_{x}\right| m\right\rangle\left\langle m\left|v_{y}\right| n\right\rangle}{\left(E_{n}-E_{m}\right)^{2}}-\frac{\left\langle n\left|v_{y}\right| m\right\rangle\left\langle m\left|v_{x}\right| n\right\rangle}{\left(E_{n}-E_{m}\right)^{2}}\right) .
\end{aligned}
$$

Recall that the system is in a periodic potential, which allows the use of the Bloch states, $\left\{\left|u_{n \mathbf{k}}\right\rangle\right\}$ as eigenstates where $n$ is the band index. Using the Heisenberg equation of motion once again, we now obtain the identity

$$
\begin{aligned}
\left\langle u_{m \mathbf{k}}\left|v_{\mu}\right| u_{n \mathbf{k}}\right\rangle & =\frac{1}{i \hbar}\left\langle u_{m \mathbf{k}}\left|\left[i \frac{\partial}{\partial k_{\mu}}, H\right]\right| u_{n \mathbf{k}}\right\rangle \\
& =\frac{1}{\hbar}\left(E_{n \mathbf{k}}-E_{m \mathbf{k}}\right)\left\langle u_{m \mathbf{k}}\left|\frac{\partial}{\partial k_{\mu}}\right| u_{n \mathbf{k}}\right\rangle
\end{aligned}
$$

where $\mathbf{k}$ is a two-dimensional vector. Thus,

$$
\sigma_{x y}=\frac{-i \hbar e^{2}}{L^{2}} \sum_{\mathbf{k}} \sum_{n} f\left(E_{n \mathbf{k}}\right) \sum_{m \neq n} \frac{1}{\hbar^{2}}\left(-\left\langle u_{n \mathbf{k}}\left|\frac{\partial}{\partial k_{x}}\right| u_{m \mathbf{k}}\right\rangle\left\langle u_{m \mathbf{k}}\left|\frac{\partial}{\partial k_{y}}\right| u_{n \mathbf{k}}\right\rangle+\left\langle u_{n \mathbf{k}}\left|\frac{\partial}{\partial k_{y}}\right| u_{m \mathbf{k}}\right\rangle\left\langle u_{m \mathbf{k}}\left|\frac{\partial}{\partial k_{x}}\right| u_{n \mathbf{k}}\right\rangle\right) .
$$

A simple integration by parts yields the identity

$$
\left\langle u_{n \mathbf{k}}\left|\frac{\partial}{\partial k_{\mu}}\right| u_{m \mathbf{k}}\right\rangle=-\left\langle\frac{\partial}{\partial k_{\mu}} u_{n \mathbf{k}} \mid u_{m \mathbf{k}}\right\rangle
$$

which implies

$$
\begin{aligned}
\sigma_{x y} & =\frac{-i e^{2}}{\hbar L^{2}} \sum_{\mathbf{k}} \sum_{n} f\left(E_{n \mathbf{k}}\right) \sum_{m \neq n}\left(\left\langle\frac{\partial}{\partial k_{x}} u_{n \mathbf{k}} \mid u_{m \mathbf{k}}\right\rangle\left\langle u_{m \mathbf{k}}\left|\frac{\partial}{\partial k_{y}}\right| u_{n \mathbf{k}}\right\rangle-\left\langle\frac{\partial}{\partial k_{y}} u_{n \mathbf{k}} \mid u_{m \mathbf{k}}\right\rangle\left\langle u_{m \mathbf{k}}\left|\frac{\partial}{\partial k_{x}}\right| u_{n \mathbf{k}}\right\rangle\right) \\
& =\frac{-i e^{2}}{\hbar L^{2}} \sum_{\mathbf{k}} \sum_{n} f\left(E_{n \mathbf{k}}\right)\left(\left\langle\frac{\partial}{\partial k_{x}} u_{n \mathbf{k}}\left|\frac{\partial}{\partial k_{y}}\right| u_{n \mathbf{k}}\right\rangle-\left\langle\frac{\partial}{\partial k_{y}} u_{n \mathbf{k}}\left|\frac{\partial}{\partial k_{x}}\right| u_{n \mathbf{k}}\right\rangle\right) .
\end{aligned}
$$

For Bloch states, the Berry connection is given by

$$
\mathbf{a}_{n}(\mathbf{k})=-i\left\langle u_{n \mathbf{k}}\left|\frac{\partial}{\partial \mathbf{k}}\right| u_{n \mathbf{k}}\right\rangle,
$$


allowing the Hall conductivity to be expressed in terms of the Berry connection,

$$
\begin{aligned}
\sigma_{x y} & =\frac{e^{2}}{\hbar L^{2}} \sum_{\mathbf{k}} \sum_{n} f\left(E_{n \mathbf{k}}\right)\left(\frac{\partial}{\partial k_{x}} a_{n, y}-\frac{\partial}{\partial k_{y}} a_{n, x}\right) \\
& =\frac{e^{2}}{\hbar L^{2}} \frac{L^{2}}{(2 \pi)^{2}} \int_{B Z} d^{2} \mathbf{k} \sum_{n} f\left(E_{n \mathbf{k}}\right)\left(\frac{\partial}{\partial k_{x}} a_{n, y}-\frac{\partial}{\partial k_{y}} a_{n, x}\right) .
\end{aligned}
$$

The Hall conductivity then reduces to

$$
\sigma_{x y}=\nu \frac{e^{2}}{h}
$$

with

$$
\nu=\sum_{n} \int_{B Z} \frac{d^{2} \mathbf{k}}{2 \pi}\left(\frac{\partial a_{n, y}}{\partial k_{x}}-\frac{\partial a_{n, x}}{\partial k_{y}}\right),
$$

where the sum over $n$ is a sum over the occupied bands. As $\nu$ is a "filling factor", we have also used

$$
f\left(E_{n \mathbf{k}}\right)= \begin{cases}1, & \forall \mathbf{k} \text { for occupied bands }\{n\} \\ 0, & \text { otherwise }\end{cases}
$$

Now write $\nu$ as $\sum_{n} \nu_{n}$ with $\nu_{n}$ being the contribution from the $n$th band

$$
\begin{aligned}
\nu_{n} & =\int_{B Z} \frac{d^{2} \mathbf{k}}{2 \pi}\left(\frac{\partial a_{n, y}}{\partial k_{x}}-\frac{\partial a_{n, x}}{\partial k_{y}}\right) \\
& =\frac{1}{2 \pi} \oint_{\partial B Z} d \mathbf{k} \cdot \mathbf{a}_{n}(\mathbf{k}) \\
& =-\frac{1}{2 \pi} \gamma_{n}[\partial B Z],
\end{aligned}
$$

where $\gamma_{n}$ is the Berry phase. The integer $\nu=\sum_{n} \nu_{n}$ is a topological invariant known as the TKNN number or the first Chern number. 


\section{Appendix B}

\section{Generalization of BdG Theory For Topolog- ICAL SUPERCONDUCTIVITY}

In Chapter 2 we discussed spin-independent BdG theory for conventional superconductivity. As the goal of this thesis is to investigate the properties of topological superconductivity with the inclusion of Rashba spin-orbit ( $\mathrm{SO}$ ) coupling and a Zeeman field, we outline the process of generalizing the $\mathrm{BdG}$ theory to the spin-dependent case. First recall the spinindependent mean-field Hamiltonian (2.36),

$$
\begin{aligned}
\mathcal{H}_{\mathrm{eff}} & =H_{0}+\sum_{i \sigma} V_{i i}^{(H)} \hat{n}_{i \sigma}+\frac{1}{2} \sum_{\langle i j\rangle \sigma} V_{i j}^{(H)} \hat{n}_{i \sigma}-\frac{1}{2} \sum_{\langle i j\rangle \sigma} V_{i j}^{(F)} \hat{c}_{i \sigma}^{\dagger} \hat{c}_{j \sigma} \\
& +\sum_{i} \Delta_{i i} \hat{c}_{i \uparrow}^{\dagger} \hat{c}_{i \downarrow}^{\dagger}+\frac{1}{2} \sum_{\langle i j\rangle} \Delta_{i j} \hat{c}_{i \uparrow}^{\dagger} \hat{c}_{j \downarrow}^{\dagger}+\text { H.c. }
\end{aligned}
$$

where $H_{0}$ is the single-particle Hamiltonian,

$$
H_{0}=\sum_{\langle i j\rangle \sigma} t_{i j} \hat{c}_{i \sigma}^{\dagger} \hat{c}_{j \sigma}+\sum_{i \sigma}\left(\epsilon_{i}-\mu\right) \hat{n}_{i \sigma}
$$

and H.c. stands for Hermitian conjugate. Here $V_{i i}^{(H)}$ and $V_{i j}^{(H)}$ are the on- and off-site Hartree potentials, $V_{i j}^{(F)}$ is the Fock potential, $\Delta_{i i}$ and $\Delta_{i j}$ are the on- and off-site order parameters, $t_{i j}$ the hopping amplitude, $\mu$ the chemical potential and lastly $\epsilon_{i}$ is a single-particle impurity potential at site $i$. As we will be considering only on-site $s$-wave interactions, from now on we neglect the off-site Hartree potential and off-site order parameter as well as the Fock potential.

As a model for topological superconductivity, in the single-particle Hamiltonian $H_{0}$ we include both Rashba spin-orbit coupling terms and Zeeman coupling terms. Hence in general $\left\langle\hat{n}_{i \uparrow}\right\rangle \neq\left\langle\hat{n}_{i \downarrow}\right\rangle$ and the Hartree potential that the spin-up electron feels is different from that felt by the spin-down electron. The Hartree potential created by the electron with spin $\sigma(=\uparrow, \downarrow)$ and felt by the electron with opposite spin $\bar{\sigma} \neq \sigma$ at site $i$ is given by

$$
V_{i i \sigma}^{(H)}=U_{i i}\left\langle\hat{n}_{i \sigma}\right\rangle=U_{i i}\left\langle c_{i \sigma}^{\dagger} c_{i \sigma}\right\rangle
$$

The $s$-wave superconducting order parameter is given (as usual) by

$$
\Delta_{i i}=U_{i i}\left\langle c_{i \downarrow} c_{i \uparrow}\right\rangle
$$

Thus, in a topological superconductor with Rashba SO and Zeeman coupling, the diagonal 
terms in the spin-dependent BdG matrix are

$$
\sum_{i \sigma}\left(\epsilon_{i \sigma}-\mu+h_{\sigma}+V_{i i \bar{\sigma}}^{(H)}\right) c_{i \sigma}^{\dagger} c_{i \sigma}
$$

where $\epsilon_{i \sigma}$ is the single-particle impurity potential at site $i$ which can depend on spin $\sigma$, and $h_{\uparrow}=-h$ and $h_{\downarrow}=h$ is the Zeeman energy. To simplify the notation in the following equations, we first omit the Rashba SO terms allowing us to write the effective Hamiltonian as

$$
\begin{aligned}
\mathcal{H}_{\mathrm{eff}} & =\sum_{\langle i j\rangle \sigma} t_{i j} c_{i \sigma}^{\dagger} c_{j \sigma}+\sum_{i \sigma}\left(\epsilon_{i \sigma}-\mu+h_{\sigma}\right) c_{i \sigma}^{\dagger} c_{i \sigma}+\sum_{i \sigma} V_{i i \bar{\sigma}}^{(H)} c_{i \sigma}^{\dagger} c_{i \sigma} \\
& +\sum_{i}\left[\Delta_{i i} c_{i \uparrow}^{\dagger} c_{i \downarrow}^{\dagger}+\left(\Delta_{i i}\right)^{*} c_{i \downarrow} c_{i \uparrow}\right]-\sum_{i} \frac{1}{U_{i i}}\left[V_{i i \uparrow}^{(H)} V_{i i \downarrow}^{(H)}+\left|\Delta_{i i}^{\downarrow \uparrow}\right|^{2}\right] .
\end{aligned}
$$

In order to write this effective Hamiltonian in matrix form, we use the anticommutation relations that the electron operators satisfy to rewrite (B.6) in the form

$$
\begin{aligned}
& \mathcal{H}_{\mathrm{eff}}=\frac{1}{2} \sum_{\langle i j\rangle \sigma} t_{i j} c_{i \sigma}^{\dagger} c_{j \sigma}-\frac{1}{2} \sum_{\langle i j\rangle \sigma} t_{i j}^{*} c_{i \sigma} c_{j \sigma}^{\dagger}+\frac{1}{2} \sum_{i \sigma}\left(\epsilon_{i \sigma}-\mu+h_{\sigma}+V_{i i \bar{\sigma}}^{(H)}\right) c_{i \sigma}^{\dagger} c_{i \sigma} \\
- & \frac{1}{2} \sum_{i \sigma}\left(\epsilon_{i \sigma}-\mu+h_{\sigma}+V_{i i \bar{\sigma}}^{(H)}\right) c_{i \sigma} c_{i \sigma}^{\dagger}+\frac{1}{2} \sum_{i \sigma}\left(\epsilon_{i \sigma}-\mu+h_{\sigma}+V_{i i \bar{\sigma}}^{(H)}\right) \\
+ & \frac{1}{2} \sum_{i}\left[\Delta_{i i} c_{i \uparrow}^{\dagger} c_{i \downarrow}^{\dagger}+\text { H.c. }\right]+\frac{1}{2} \sum_{i}\left[-\Delta_{i i} c_{i \downarrow}^{\dagger} c_{i \uparrow}^{\dagger}+\text { H.c. }\right]-\sum_{i} \frac{1}{U_{i i}}\left[V_{i i \uparrow}^{(H)} V_{i i \downarrow}^{(H)}+\left.\left|\Delta_{i i}\right|\right|^{2} \text { B.. } 7\right)
\end{aligned}
$$

We now add in the Rashba terms denoted as $V_{i j}^{\mathrm{SO}}$ and write the Hamiltonian (B.7) in matrix form as

$$
\begin{aligned}
\mathcal{H}_{\mathrm{eff}} & =\frac{1}{2} \sum_{i j}\left(c_{i \uparrow}^{\dagger} c_{i \downarrow}^{\dagger} c_{i \uparrow} c_{i \downarrow}\right)\left(\begin{array}{cccc}
t_{i j}+V_{i i \uparrow}^{\mathrm{diag}} & \left(V_{i j}^{\mathrm{SO}}\right)^{\dagger} & 0 & \Delta_{i i} \\
V_{i j}^{\mathrm{SO}} & t_{i j}+V_{i i \downarrow}^{\mathrm{diag}} & -\Delta_{i i} & 0 \\
0 & -\Delta_{i i}^{*} & -t_{i j}^{*}-V_{i i \uparrow}^{\mathrm{diag}} & -\left(V_{i j}^{\mathrm{SO}}\right)^{T} \\
\Delta_{i i}^{*} & 0 & -\left(V_{i j}^{\mathrm{SO}}\right)^{*} & -t_{i j}^{*}-V_{i i \downarrow}^{\mathrm{diag}}
\end{array}\right)\left(\begin{array}{c}
c_{j \uparrow} \\
c_{j \downarrow} \\
c_{j \uparrow}^{\dagger} \\
c_{j \downarrow}^{\dagger}
\end{array}\right) \\
& +\frac{1}{2} \sum_{i \sigma}\left(\epsilon_{i \sigma}-\mu+h_{\sigma}+V_{i i \bar{\sigma}}^{(H)}\right)-\sum_{i} \frac{1}{U_{i i}}\left[V_{i i \uparrow}^{(H)} V_{i i \downarrow}^{(H)}+\left|\Delta_{i i}^{\downarrow \uparrow}\right|^{2}\right]
\end{aligned}
$$

where $V_{i i \sigma}^{\text {diag }}=\epsilon_{i \sigma}-\mu+h_{\sigma}+V_{i i \bar{\sigma}}^{(H)}$ and the Kronecker delta $\delta_{i j}$ is implicit for $V_{i i \sigma}^{\text {diag }}$ and $\Delta_{i i}$. Next, define the average spin-up and spin-down Hartree potentials by

$$
\bar{V}_{\uparrow}^{(H)}=\frac{1}{N} \sum_{i} V_{i i \uparrow}^{(H)}, \quad \bar{V}_{\downarrow}^{(H)}=\frac{1}{N} \sum_{i} V_{i i \downarrow}^{(H)},
$$

where $N$ is the number of lattice sites. We measure the chemical potential from the average 
of these Hartree potentials and define $\tilde{\mu}$ by

$$
\tilde{\mu}=\mu-\frac{\bar{V}_{\uparrow}^{(H)}+\bar{V}_{\downarrow}^{(H)}}{2} .
$$

To obtain a desired filling factor for the system, we specify the electron density $n_{e}$ and solve for $\tilde{\mu}$ as a root of

$$
F(\tilde{\mu})=\frac{1}{N} \sum_{i \sigma}\left\langle c_{i \sigma}^{\dagger} c_{i \sigma}\right\rangle-n_{e} .
$$

Then, by defining

$$
\delta \bar{V}=\frac{\bar{V}_{\uparrow}^{(H)}-\bar{V}_{\downarrow}^{(H)}}{2},
$$

we rewrite the diagonal elements for spin up and down as

$$
\begin{aligned}
& V_{i i \uparrow}^{\text {diag }}=\epsilon_{i \uparrow}-\tilde{\mu}-\delta \bar{V}-h+V_{i i \downarrow}^{(H)}-\bar{V}_{\downarrow}^{(H)}, \\
& V_{i i \downarrow}^{\text {diag }}=\epsilon_{i \downarrow}-\tilde{\mu}+\delta \bar{V}+h+V_{i i \uparrow}^{(H)}-\bar{V}_{\uparrow}^{(H)},
\end{aligned}
$$

and the BdG matrix in (B.8) reduces to

$$
\begin{aligned}
\mathcal{H}_{\mathrm{eff}} & =\frac{1}{2} \sum_{i j}\left(c_{i \uparrow}^{\dagger} c_{i \downarrow}^{\dagger} c_{i \uparrow} c_{i \downarrow}\right)\left(\begin{array}{cccc}
t_{i j}+V_{i i \uparrow}^{\mathrm{diag}} & \left(V_{i j}^{\mathrm{SO}}\right)^{\dagger} & 0 & \Delta_{i i} \\
V_{i j}^{\mathrm{SO}} & t_{i j}+V_{i i \downarrow}^{\mathrm{diag}} & -\Delta_{i i} & 0 \\
0 & -\Delta_{i i}^{*} & -t_{i j}^{*}-V_{i i \uparrow}^{\mathrm{diag}} & -\left(V_{i j}^{\mathrm{SO}}\right)^{T} \\
\Delta_{i i}^{*} & 0 & -\left(V_{i j}^{\mathrm{SO}}\right)^{*} & -t_{i j}^{*}-V_{i i \downarrow}^{\mathrm{diag}}
\end{array}\right)\left(\begin{array}{c}
c_{j \uparrow} \\
c_{j \downarrow} \\
c_{j \uparrow}^{\dagger} \\
c_{j \downarrow}^{\dagger}
\end{array}\right) \\
& +\frac{1}{2} \sum_{i \sigma}\left(\epsilon_{i \sigma}-\tilde{\mu}+\delta \bar{V}_{\sigma}+h_{\sigma}+V_{i i \bar{\sigma}}^{(H)}-\bar{V}_{\bar{\sigma}}^{(H)}\right)-\sum_{i} \frac{1}{U_{i i}}\left[V_{i i \uparrow}^{(H)} V_{i i \downarrow}^{(H)}+\left|\Delta_{i i}^{\downarrow \uparrow}\right|^{2}\right],
\end{aligned}
$$

where $\delta \bar{V}_{\sigma}=-\delta \bar{V}$ for $\sigma=\uparrow$ and $\delta \bar{V}_{\sigma}=\delta \bar{V}$ for $\sigma=\downarrow$. Typically there are more spin-up electrons than spin-down electrons, namely,

$$
\frac{1}{N} \sum_{i}\left\langle c_{i \uparrow}^{\dagger} c_{i \uparrow}\right\rangle>\frac{1}{N} \sum_{i}\left\langle c_{i \downarrow}^{\dagger} c_{i \downarrow}\right\rangle .
$$

From (B.9) this implies $\delta \bar{V}<0$ which effectively reduces the Zeeman field. Intuitively, the average energy gain by electron-phonon coupling with spin-up electrons makes the electron to have its spin down less costly in terms of the Zeeman energy. Define the effective Zeeman field,

$$
\tilde{h}_{\sigma} \equiv h_{\sigma}+\delta \bar{V}_{\sigma}
$$

so that

$$
V_{i i \sigma}^{\operatorname{diag}}=\epsilon_{i \sigma}-\tilde{\mu}-\tilde{h}_{\sigma}+V_{i i \bar{\sigma}}^{(H)}-\bar{V}_{\bar{\sigma}}^{(H)}
$$

When the system has translational symmetry, with $\bar{V}_{\downarrow}^{(H)}=V_{i i \downarrow}^{(H)}, \bar{V}_{\uparrow}^{(H)}=V_{i i \uparrow}^{(H)} ; \forall i$, the diag- 
onal elements of the effective Hamiltonian in Eq. (B.6) reduce to

$$
\begin{aligned}
& \sum_{\sigma} \sum_{i}\left(-\mu+h_{\sigma}+\bar{V}_{\bar{\sigma}}^{(H)}\right) c_{i \sigma}^{\dagger} c_{i \sigma}=\sum_{\sigma} \sum_{\boldsymbol{k}}\left(-\mu+h_{\sigma}+\bar{V}_{\bar{\sigma}}^{(H)}\right) c_{\boldsymbol{k} \sigma}^{\dagger} c_{\boldsymbol{k} \sigma} \\
= & \sum_{\boldsymbol{k}}\left[(-\tilde{\mu}-\tilde{h}) c_{\boldsymbol{k} \uparrow}^{\dagger} c_{\boldsymbol{k} \uparrow}+(-\tilde{\mu}+\tilde{h}) c_{\boldsymbol{k} \downarrow}^{\dagger} c_{\boldsymbol{k} \downarrow}\right] .
\end{aligned}
$$

This implies that when the Hartree potential is taken into account, the different topological regions as classified in Table I of Sato et al. [55] must be altered by replacing $\mu$ by $\tilde{\mu}$, and $\mu_{\mathrm{B}} H_{z}$ by $\tilde{h}$. In the notation of Table $5.1, h$ is replaced by $\tilde{h}$.

Lastly, we diagonalize the BdG matrix (B.15) by means of a unitary transformation:

$$
\begin{aligned}
\mathcal{H}_{\mathrm{eff}} & =\frac{1}{2} \sum_{n m}\left(\gamma_{n 1}^{\dagger} \gamma_{n 2}^{\dagger} \gamma_{n 1} \gamma_{n 2}\right)\left(\begin{array}{cccc}
E_{n 1} \delta_{n m} & 0 & 0 & 0 \\
0 & E_{n 2} \delta_{n m} & 0 & 0 \\
0 & 0 & -E_{n 1} \delta_{n m} & 0 \\
0 & 0 & 0 & -E_{n 2} \delta_{n m}
\end{array}\right)\left(\begin{array}{c}
\gamma_{m 1} \\
\gamma_{m 2} \\
\gamma_{m 1}^{\dagger} \\
\gamma_{m 2}^{\dagger}
\end{array}\right) \\
& +\frac{1}{2} \sum_{i \sigma}\left(\epsilon_{i \sigma}-\tilde{\mu}+\tilde{h}_{\sigma}+V_{i i \bar{\sigma}}^{(H)}-\bar{V}_{\bar{\sigma}}^{(H)}\right)-\sum_{i} \frac{1}{U_{i i}}\left[V_{i i \uparrow}^{(H)} V_{i i \downarrow}^{(H)}+\left|\Delta_{i i}^{\downarrow \uparrow}\right|^{2}\right],
\end{aligned}
$$

The sum over $n$ and $m$ is

$$
\begin{aligned}
& \frac{1}{2} \sum_{n}\left(E_{n 1} \gamma_{n 1}^{\dagger} \gamma_{n 1}+E_{n 2} \gamma_{n 2}^{\dagger} \gamma_{n 2}-E_{n 1} \gamma_{n 1} \gamma_{n 1}^{\dagger}-E_{n 2} \gamma_{n 2} \gamma_{n 2}^{\dagger}\right) \\
= & \sum_{n}\left(E_{n 1} \gamma_{n 1}^{\dagger} \gamma_{n 1}+E_{n 2} \gamma_{n 2}^{\dagger} \gamma_{n 2}\right)-\frac{1}{2} \sum_{n}\left(E_{n 1}+E_{n 2}\right) .
\end{aligned}
$$

At zero temperature, $\left\langle\gamma_{n \alpha}^{\dagger} \gamma_{n \alpha}\right\rangle=0$. Hence the ground-state energy $E=\left\langle\mathcal{H}_{\mathrm{eff}}\right\rangle$ is

$$
E=-\frac{1}{2} \sum_{n}\left(E_{n 1}+E_{n 2}\right)+\frac{1}{2} \sum_{i \sigma}\left(\epsilon_{i \sigma}-\tilde{\mu}+V_{i i \bar{\sigma}}^{(H)}-\bar{V}_{\bar{\sigma}}^{(H)}\right)-\sum_{i} \frac{1}{U_{i i}}\left[V_{i i \uparrow}^{(H)} V_{i i \downarrow}^{(H)}+\left|\Delta_{i i}^{\downarrow \uparrow}\right|^{2}\right] .
$$




\section{Appendix C}

\section{Equivalence of the Hamiltonian of TSC AND THE Hamiltonian OF TCDW}

In this Appendix, we aim to show that the Hamiltonian of TSC given by (5.9) is formally equivalent to the Hamiltonian of TCDW given by (6.3). We begin with the normal-state Hamiltonian

$$
\begin{aligned}
\mathcal{H}_{N} & =\sum_{\mathbf{k}, \sigma} \epsilon(\mathbf{k}) c_{\mathbf{k} \sigma}^{\dagger} c_{\mathbf{k} \sigma}-h \sum_{\mathbf{k}, \sigma, \sigma^{\prime}}\left(\sigma_{z}\right)_{\sigma \sigma^{\prime}} c_{\mathbf{k} \sigma}^{\dagger} c_{\mathbf{k} \sigma^{\prime}}+\alpha \sum_{\mathbf{k}, \sigma, \sigma^{\prime}} \mathcal{L}(\mathbf{k}) \cdot \boldsymbol{\sigma}_{\sigma \sigma^{\prime}} c_{\mathbf{k} \sigma}^{\dagger} c_{\mathbf{k} \sigma^{\prime}} \\
& =\mathcal{H}_{0}+\mathcal{H}_{Z}+\mathcal{H}_{S O},
\end{aligned}
$$

where $c_{\mathbf{k} \sigma}^{\dagger}\left(c_{\mathbf{k} \sigma}\right)$ is the creation (annihilation) operator for an electron with momentum $\mathbf{k}=$ $\left(k_{x}, k_{y}\right)$ and spin $\sigma, \epsilon(\mathbf{k})=-2 t\left(\cos k_{x}+\cos k_{y}\right)-\mu$ is the energy dispersion with hopping parameter $t$ and chemical potential $\mu, \alpha \mathcal{L}(\mathbf{k})=\alpha\left(\sin k_{y},-\sin k_{x}\right)$ represents the Rashba spin-orbit coupling, $h$ is the Zeeman field and lastly $\sigma_{i}(i=x, y, z)$ are the Pauli matrices. To obtain the Hamiltonian for TSC, we introduce the $s$-wave superconducting order parameter

$$
\Delta_{S}=\left\langle c_{-\mathbf{k} \downarrow} c_{\mathbf{k} \uparrow}\right\rangle
$$

and define

$$
\Delta=i \Delta_{S} \sigma_{y}
$$

Then we have

$$
\mathcal{H}_{S C}=\mathcal{H}_{N}+\mathcal{H}_{S}
$$

where

$$
\mathcal{H}_{S}=\sum_{\mathbf{k}, \sigma, \sigma^{\prime}}\left(\Delta c_{\mathbf{k} \sigma}^{\dagger} c_{-\mathbf{k} \sigma^{\prime}}^{\dagger}+\Delta^{*} c_{-\mathbf{k} \sigma} c_{\mathbf{k} \sigma^{\prime}}\right)
$$

In order to write the TSC Hamiltonian in matrix form, we rewrite each term as follows, using the kinetic term as an example,

$$
\begin{aligned}
\mathcal{H}_{0} & =\frac{1}{2} \sum_{\mathbf{k}, \sigma}\left[\epsilon(\mathbf{k}) c_{\mathbf{k} \sigma}^{\dagger} c_{\mathbf{k} \sigma}-\epsilon(\mathbf{k}) c_{\mathbf{k} \sigma} c_{\mathbf{k} \sigma}^{\dagger}\right]+\frac{1}{2} \sum_{\mathbf{k}} \epsilon(\mathbf{k}) \\
& =\frac{1}{2} \sum_{\mathbf{k}, \sigma}\left[\epsilon(\mathbf{k}) c_{\mathbf{k} \sigma}^{\dagger} c_{\mathbf{k} \sigma}-\epsilon(-\mathbf{k}) c_{-\mathbf{k} \sigma} c_{-\mathbf{k} \sigma}^{\dagger}\right]+\frac{1}{2} \sum_{\mathbf{k}} \epsilon(\mathbf{k}),
\end{aligned}
$$

where in the first equality we have used $\left\{c_{\mathbf{k} \sigma}^{\dagger}, c_{\mathbf{k}^{\prime} \sigma^{\prime}}\right\}=\delta_{\sigma \sigma^{\prime}} \delta_{\mathbf{k k}^{\prime}}$ and in the second equality we have relabeled $\mathbf{k} \rightarrow-\mathbf{k}$ in the second term. Performing such an expansion on all remaining 
terms we obtain the Hamiltonian for topological superconductivity (5.9):

$$
\mathcal{H}_{S C}=\frac{1}{2} \sum_{\mathbf{k}}\left(c_{\mathbf{k} \uparrow}^{\dagger} c_{\mathbf{k} \downarrow}^{\dagger} c_{-\mathbf{k} \uparrow} c_{-\mathbf{k} \downarrow}\right) \mathcal{H}_{\mathrm{BdG}}(\mathbf{k})\left(\begin{array}{c}
c_{\mathbf{k} \uparrow} \\
c_{\mathbf{k} \downarrow} \\
c_{-\mathbf{k} \uparrow}^{\dagger} \\
c_{-\mathbf{k} \downarrow}^{\dagger}
\end{array}\right)
$$

where

$$
\mathcal{H}_{\mathrm{BdG}}(\mathbf{k})=\left(\begin{array}{cc}
\epsilon(\mathbf{k})-h \sigma_{z}+\alpha \mathcal{L}(\mathbf{k}) \cdot \boldsymbol{\sigma} & i \Delta_{S} \sigma_{y} \\
i \Delta_{S}^{*} \sigma_{y} & -\epsilon(\mathbf{k})+h \sigma_{z}+\alpha \mathcal{L}(\mathbf{k}) \cdot \boldsymbol{\sigma}
\end{array}\right),
$$

with $\mathcal{L}(\mathbf{k}) \cdot \boldsymbol{\sigma}=\sigma_{x} \sin k_{y}-\sigma_{y} \sin k_{x}$. We note also that (C.7) contains an additional constant term that is dropped by convention. Upon diagonalizing the Hamiltonian (C.8), we then obtain the bulk spectrum,

$$
E_{ \pm}(\mathbf{k})=\sqrt{\epsilon(\mathbf{k})^{2}+\alpha^{2}|\mathcal{L}(\mathbf{k})|^{2}+h^{2}+\left|\Delta_{S}\right|^{2} \pm 2 \xi(\mathbf{k})}
$$

where $\xi(\mathbf{k})=\sqrt{\epsilon(\mathbf{k})^{2} \alpha^{2}|\mathcal{L}(\mathbf{k})|^{2}+\left(\epsilon(\mathbf{k})^{2}+\left|\Delta_{S}\right|^{2}\right) h^{2}}$.

To obtain the BdG Hamiltonian for TCDW, we begin with the normal-state Hamiltonian $\mathcal{H}_{N}$ and introduce the CDW order parameter,

$$
\Delta_{C}=\left\langle c_{\mathbf{k} \uparrow}^{\dagger} c_{\mathbf{k}+\mathbf{Q} \uparrow}\right\rangle=\left\langle c_{\mathbf{k} \downarrow}^{\dagger} c_{\mathbf{k}+\mathbf{Q} \downarrow}\right\rangle,
$$

so that

$$
\mathcal{H}_{C D W}=\mathcal{H}_{N}+\mathcal{H}_{C},
$$

where

$$
\mathcal{H}_{C}=\sum_{\mathbf{k}, \sigma} \Delta_{C} c_{\mathbf{k} \sigma}^{\dagger} c_{\mathbf{k}+\mathbf{Q} \sigma}
$$

This time, we first rewrite $\mathcal{H}_{C D W}$ as follows, using the kinetic energy term as an example:

$$
\begin{aligned}
& \mathcal{H}_{0}=\frac{1}{2} \sum_{\mathbf{k}, \sigma}\left[\epsilon(\mathbf{k}) c_{\mathbf{k} \sigma}^{\dagger} c_{\mathbf{k} \sigma}+\epsilon(\mathbf{k}+\mathbf{Q}) c_{\mathbf{k}+\mathbf{Q} \sigma}^{\dagger} c_{\mathbf{k}+\mathbf{Q} \sigma}\right] \\
& =\frac{1}{2} \sum_{\mathbf{k}}\left[\epsilon(\mathbf{k})\left(c_{\mathbf{k} \uparrow}^{\dagger} c_{\mathbf{k} \downarrow}^{\dagger}\right) \sigma_{0}\left(\begin{array}{c}
c_{\mathbf{k} \uparrow} \\
c_{\mathbf{k} \downarrow}
\end{array}\right)+\epsilon(\mathbf{k}+\mathbf{Q})\left(c_{\mathbf{k}+\mathbf{Q} \uparrow}^{\dagger} c_{\mathbf{k}+\mathbf{Q} \downarrow}^{\dagger}\right) \sigma_{0}\left(\begin{array}{c}
c_{\mathbf{k}+\mathbf{Q} \uparrow} \\
c_{\mathbf{k}+\mathbf{Q} \downarrow}
\end{array}\right)\right],
\end{aligned}
$$

where $\sigma_{0}=\mathbb{1}$, the identity matrix. Hence the remaining terms in $\mathcal{H}_{C D W}$ become

$$
\begin{aligned}
\mathcal{H}_{Z} & =-\frac{h}{2} \sum_{\mathbf{k}}\left[\left(\begin{array}{c}
c_{\mathbf{k} \uparrow}^{\dagger} c_{\mathbf{k} \downarrow}^{\dagger}
\end{array}\right) \sigma_{z}\left(\begin{array}{c}
c_{\mathbf{k} \uparrow} \\
c_{\mathbf{k} \downarrow}
\end{array}\right)+\left(c_{\mathbf{k}+\mathbf{Q} \uparrow}^{\dagger} c_{\mathbf{k}+\mathbf{Q} \downarrow}^{\dagger}\right) \sigma_{z}\left(\begin{array}{c}
c_{\mathbf{k}+\mathbf{Q} \uparrow} \\
c_{\mathbf{k}+\mathbf{Q} \downarrow}
\end{array}\right)\right] \\
\mathcal{H}_{S O} & =\frac{\alpha}{2} \sum_{\mathbf{k}}\left[\left(c_{\mathbf{k} \uparrow}^{\dagger} c_{\mathbf{k} \downarrow}^{\dagger}\right) \mathcal{L}(\mathbf{k}) \cdot \boldsymbol{\sigma}\left(\begin{array}{c}
c_{\mathbf{k} \uparrow} \\
c_{\mathbf{k} \downarrow}
\end{array}\right)+\left(c_{\mathbf{k}+\mathbf{Q} \uparrow}^{\dagger} c_{\mathbf{k}+\mathbf{Q} \downarrow}^{\dagger}\right) \mathcal{L}(\mathbf{k}+\mathbf{Q}) \cdot \boldsymbol{\sigma}\left(\begin{array}{c}
c_{\mathbf{k}+\mathbf{Q} \uparrow} \\
c_{\mathbf{k}+\mathbf{Q} \downarrow}
\end{array}\right)\right]
\end{aligned}
$$




$$
\mathcal{H}_{C}=\frac{\Delta_{C}}{2} \sum_{\mathbf{k}}\left[\left(c_{\mathbf{k} \uparrow}^{\dagger} c_{\mathbf{k} \downarrow}^{\dagger}\right) \sigma_{0}\left(\begin{array}{c}
c_{\mathbf{k}+\mathbf{Q} \uparrow} \\
c_{\mathbf{k}+\mathbf{Q} \downarrow}
\end{array}\right)+\left(c_{\mathbf{k}+\mathbf{Q} \uparrow}^{\dagger} c_{\mathbf{k}+\mathbf{Q} \downarrow}^{\dagger}\right) \sigma_{0}\left(\begin{array}{c}
c_{\mathbf{k} \uparrow} \\
c_{\mathbf{k} \downarrow}
\end{array}\right)\right] .
$$

In order to exactly reproduce Hamiltonian (6.3), we must switch our basis to

$$
\begin{gathered}
\left(\begin{array}{c}
-c_{\mathbf{k}+\mathbf{Q} \downarrow} \\
c_{\mathbf{k}+\mathbf{Q} \uparrow}
\end{array}\right)=-i \sigma_{y}\left(\begin{array}{c}
c_{\mathbf{k}+\mathbf{Q} \uparrow} \\
c_{\mathbf{k}+\mathbf{Q} \downarrow}
\end{array}\right), \\
\left(\begin{array}{c}
c_{\mathbf{k}+\mathbf{Q} \uparrow} \\
c_{\mathbf{k}+\mathbf{Q} \downarrow}
\end{array}\right)=i \sigma_{y}\left(\begin{array}{c}
-c_{\mathbf{k}+\mathbf{Q} \downarrow} \\
c_{\mathbf{k}+\mathbf{Q} \uparrow}
\end{array}\right) .
\end{gathered}
$$

Upon inserting (C.17) and (C.18) into (C.13-16), and performing the multiplication of the Pauli matrices, we obtain our Hamiltonian $\mathcal{H}_{C D W}$ which can be written in matrix form as

$$
\mathcal{H}_{C D W}=\frac{1}{2} \sum_{\mathbf{k}}\left(c_{\mathbf{k} \uparrow}^{\dagger} c_{\mathbf{k} \downarrow}^{\dagger}-c_{\mathbf{k}+\mathbf{Q} \downarrow}^{\dagger} c_{\mathbf{k}+\mathbf{Q} \uparrow}^{\dagger}\right) \mathcal{H}_{\mathrm{BdG}}(\mathbf{k})\left(\begin{array}{c}
c_{\mathbf{k} \uparrow} \\
c_{\mathbf{k} \downarrow} \\
-c_{\mathbf{k}+\mathbf{Q} \downarrow} \\
c_{\mathbf{k}+\mathbf{Q} \uparrow}
\end{array}\right)
$$

where

$$
\mathcal{H}_{\mathrm{BdG}}(\mathbf{k})=\left(\begin{array}{cc}
\epsilon(\mathbf{k})-h \sigma_{z}+\alpha \mathcal{L}(\mathbf{k}) \cdot \boldsymbol{\sigma} & i \Delta_{C} \sigma_{y} \\
-i \Delta_{C} \sigma_{y} & \epsilon(\mathbf{k}+\mathbf{Q})+h \sigma_{z}-\alpha \mathcal{L}(\mathbf{k}+\mathbf{Q}) \cdot \boldsymbol{\sigma}^{*}
\end{array}\right) .
$$

Then if we have $\epsilon(\mathbf{k}+\mathbf{Q})=-\epsilon(\mathbf{k})$ and $\mathcal{L}(\mathbf{k}+\mathbf{Q})=-\mathcal{L}(\mathbf{k})$, we find that the Hamiltonian for topological charge density waves (C.20) is equivalent to the Hamiltonian of topological superconductivity (C.8). 